\title{
Methods for analysis of brain connectivity : An IFCN-sponsored review
}

\author{
Rossini, P. M.
}

2019-10

Rossini , P M , Di lorio , R, Bentivoglio , M , Bertini , G, Ferreri , F , Gerloff , C , Ilmoniemi , R J , Miraglia, F , Nitsche, M A, Pestilli, F, Rosanova, M , Shirota , Y, Tesoriero, C , Ugawa , Y , Vecchio , F , Ziemann , U \& Hallett , M 2019, ' 'Methods for analysis of brain connectivity : An IFCN-sponsored review ', Clinical Neurophysiology , vol. 130 , no. 10 , pp. 1833-1858 . https://doi.org/10.1016/j.clinph.2019.06.006

http://hdl.handle.net/10138/320954

https://doi.org/10.1016/j.clinph.2019.06.006

publishedVersion

Downloaded from Helda, University of Helsinki institutional repository.

This is an electronic reprint of the original article.

This reprint may differ from the original in pagination and typographic detail.

Please cite the original version. 
Review

\title{
Methods for analysis of brain connectivity: An IFCN-sponsored review
}

\author{
P.M. Rossini ${ }^{\mathrm{a}, \mathrm{b}, \mathrm{c}}$, R. Di Iorio ${ }^{\mathrm{c}, *}$, M. Bentivoglio ${ }^{\mathrm{d}}$, , G. Bertini $^{\mathrm{d}}$, F. Ferreri ${ }^{\mathrm{e}, \mathrm{f}}$, C. Gerloff $^{\mathrm{g}}$, \\ R.J. Ilmoniemi ${ }^{\text {h,i }}$, F. Miraglia ${ }^{\mathrm{a}, \mathrm{b}}$, M.A. Nitsche ${ }^{\mathrm{j}, \mathrm{k}}$, F. Pestilli $^{1}$, M. Rosanova ${ }^{\mathrm{m}, \mathrm{n}}$, Y. Shirota ${ }^{\mathrm{o}}$, \\ C. Tesoriero $^{\mathrm{d}}$, Y. Ugawa $^{\mathrm{P}}$, F. Vecchio ${ }^{\mathrm{b}}$, U. Ziemann ${ }^{\mathrm{q}}$, M. Hallett ${ }^{\mathrm{r}}$
}

a Institute of Neurology, Area of Neuroscience, Catholic University of Sacred Heart, Rome, Italy

${ }^{\mathrm{b}}$ Brain Connectivity Lab, IRCCS San Raffaele Pisana, Rome, Italy

${ }^{\mathrm{c}}$ Neurology Unit, IRCCS Polyclinic A. Gemelli Foundation, Rome, Italy

${ }^{\mathrm{d}}$ Department of Neuroscience, Biomedicine and Movement Sciences, University of Verona, Verona, Italy

e Department of Neuroscience, University of Padua, Padua, Italy

${ }^{\mathrm{f}}$ Department of Clinical Neurophysiology, Kuopio University Hospital, Kuopio, Finland

${ }^{\mathrm{g}}$ Department of Neurology, University Medical Center Hamburg-Eppendorf, Germany

${ }^{\mathrm{h}}$ Department of Neuroscience and Biomedical Engineering, Aalto University School of Science, Espoo, Finland

i BioMag Laboratory, HUS Medical Imaging Center, University of Helsinki and Helsinki University Hospital, Helsinki, Finland

${ }^{\mathrm{j}}$ Leibniz Research Centre for Working Environment and Human Factors, Department of Psychology and Neurosciences, Dortmund, Germany

${ }^{\mathrm{k}}$ University Medical Hospital Bergmannsheil, Department of Neurology, Bochum, Germany

${ }^{1}$ Department of Psychological and Brain Sciences, Indiana University, Bloomington, IN, USA

${ }^{\mathrm{m}}$ Department of Biomedical and Clinical Sciences "L. Sacco", University of Milan, Milan, Italy

${ }^{n}$ Fondazione Europea di Ricerca Biomedica, FERB Onlus, Milan, Italy

${ }^{\circ}$ Department of Neurology, The University of Tokyo, Tokyo, Japan

${ }^{\mathrm{p}}$ Department of Neuro-Regeneraion, Fukushima Medical University, Fukushima, Japan

${ }^{\mathrm{q}}$ Department of Neurology \& Stroke and Hertie-Institute for Clinical Brain Research, Eberhard-Karls University of Tübingen, Tübingen, Germany

${ }^{\mathrm{r}}$ Human Motor Control Section, National Institute of Neurological Disorders and Stroke, NIH, Bethesda, MD, USA

\section{A R T I C L E I N F O}

\section{Article history:}

Accepted 18 June 2019

Available online 2 July 2019

\section{Keywords:}

Brain connectivity

Anatomical connectivity

Functional connectivity

Effective connectivity

Human brain connectome

Connectomics

fMRI

EEG

\section{H I G H L I G H T S}

- There are a variety of technologies valuable for exploring human brain connectivity.

- The main aspects of anatomical, functional and effective connectivity are described.

- A multimodality approach can be useful to evaluate the human brain connectome.

\section{A B S T R A C T}

The goal of this paper is to examine existing methods to study the "Human Brain Connectome" with a specific focus on the neurophysiological ones. In recent years, a new approach has been developed to evaluate the anatomical and functional organization of the human brain: the aim of this promising multimodality effort is to identify and classify neuronal networks with a number of neurobiologically

Abbreviations: BOLD, Blood Oxygenation Level Dependent; CBI, cerebellar inhibition; CLARITY, clear lipid-exchanged acrylamide-hybridized rigid imaging/immunostain

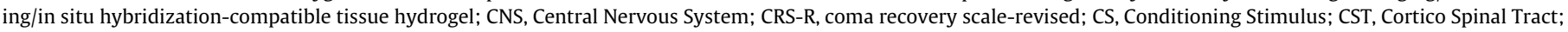

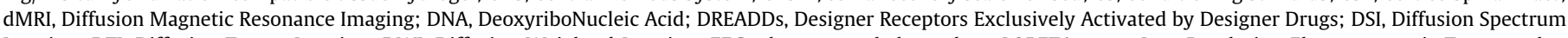

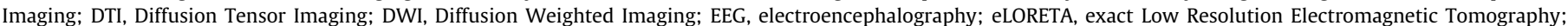

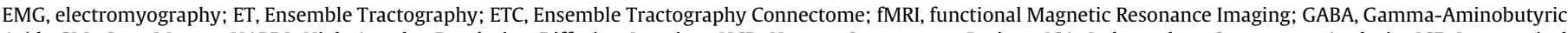

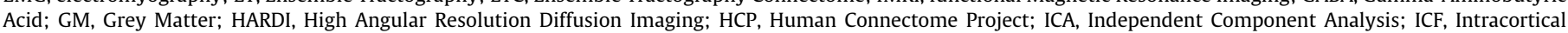

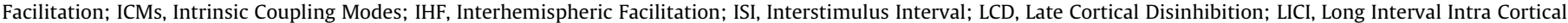

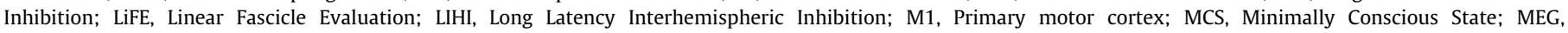

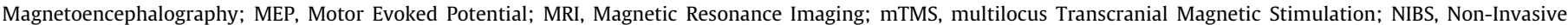

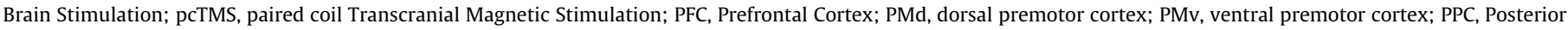

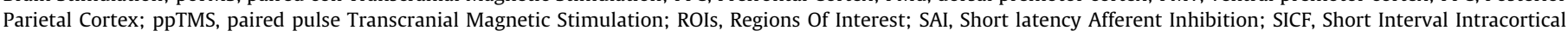

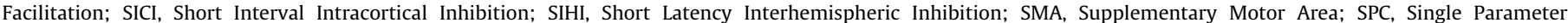

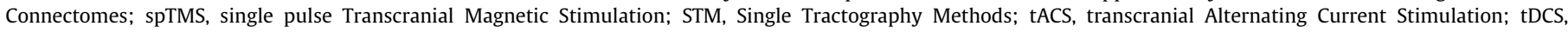

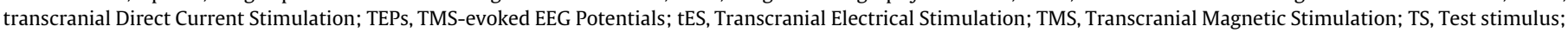
UWS, Unresponsive Wakefulness Syndrome; VS, Vegetative Wakefulness Syndrome; WM, White Matter.

* Corresponding author.

E-mail address: r.diiorio@live.it (R. Di Iorio). 
MEG

TMS-EEG

Non-invasive brain stimulation meaningful and easily computable measures to create its connectome. By defining anatomical and functional connections of brain regions on the same map through an integrated approach, comprising both modern neurophysiological and neuroimaging (i.e. flow/metabolic) brain-mapping techniques, network analysis becomes a powerful tool for exploring structural-functional connectivity mechanisms and for revealing etiological relationships that link connectivity abnormalities to neuropsychiatric disorders. Following a recent IFCN-endorsed meeting, a panel of international experts was selected to produce this current state-of-art document, which covers the available knowledge on anatomical and functional connectivity, including the most commonly used structural and functional MRI, EEG, MEG and non-invasive brain stimulation techniques and measures of local and global brain connectivity.

(c) 2019 Published by Elsevier B.V. on behalf of International Federation of Clinical Neurophysiology.

\section{Contents}

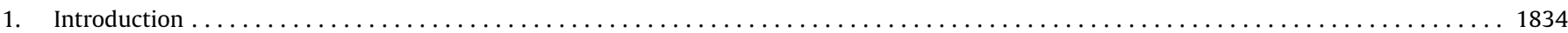

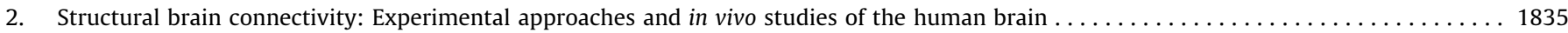

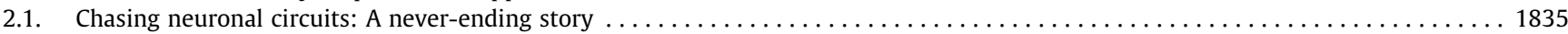

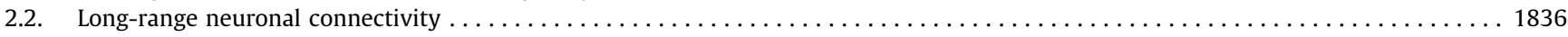

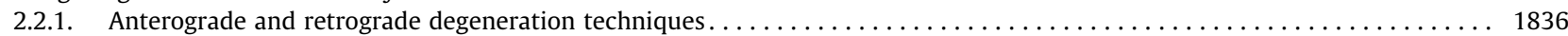

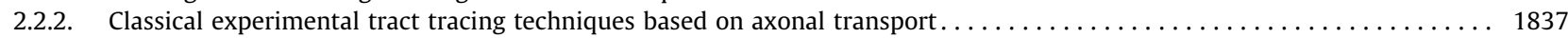

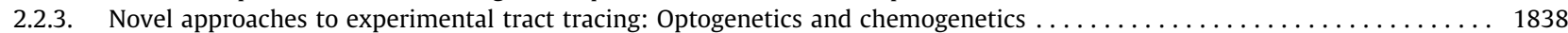

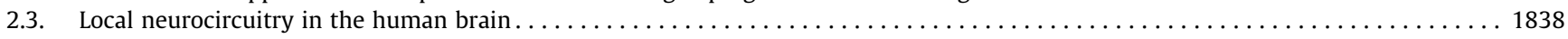

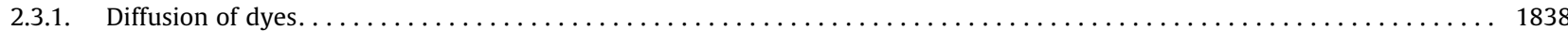

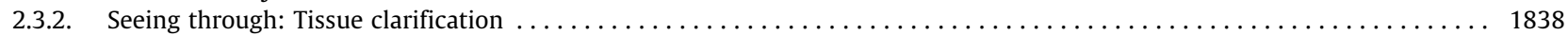

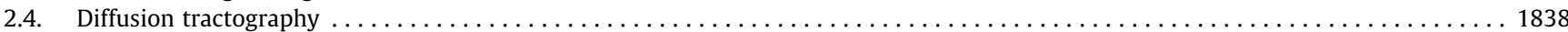

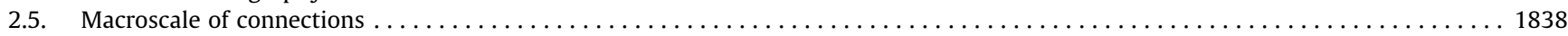

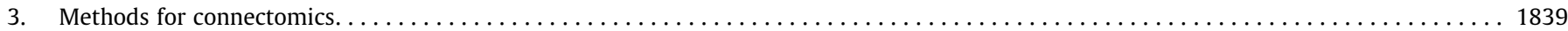

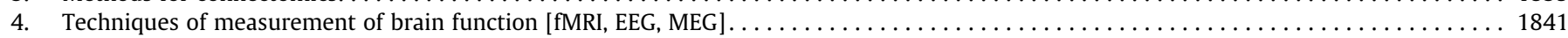

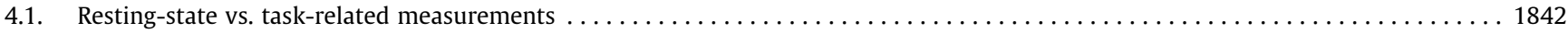

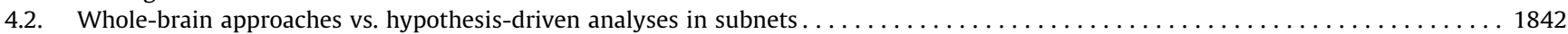

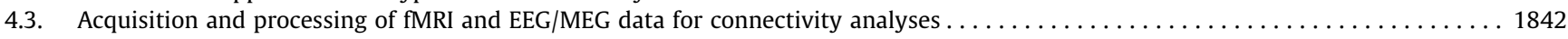

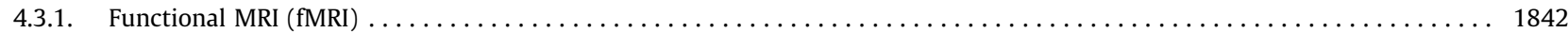

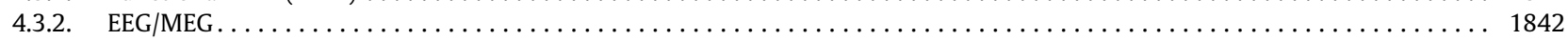

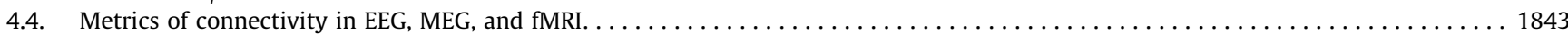

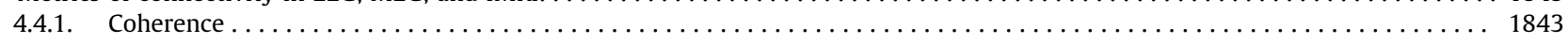

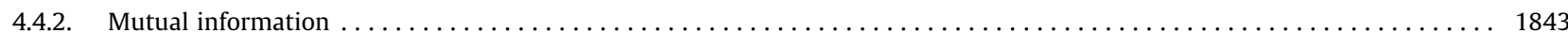

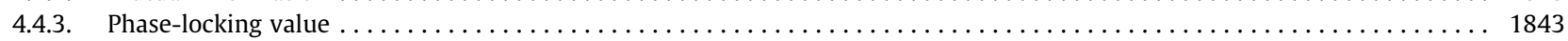

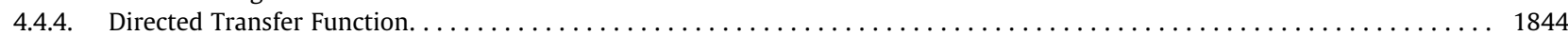

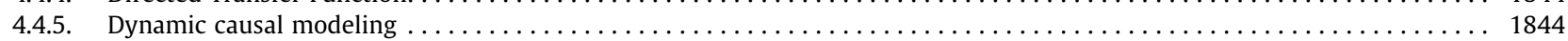

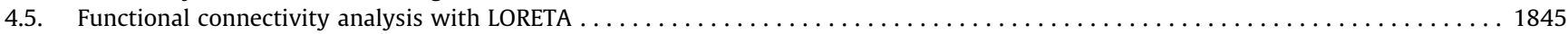

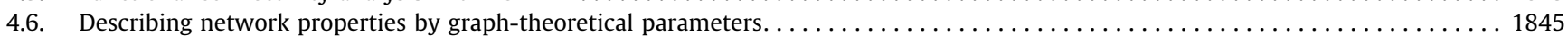

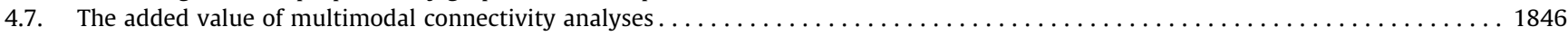

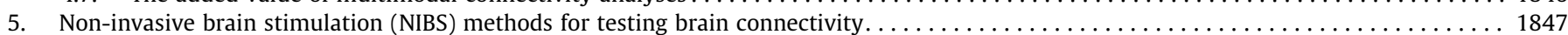

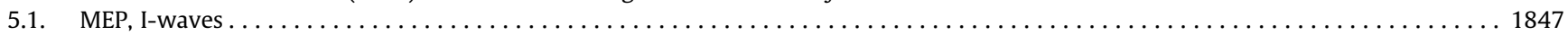

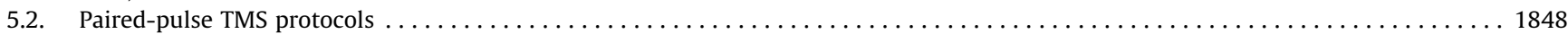

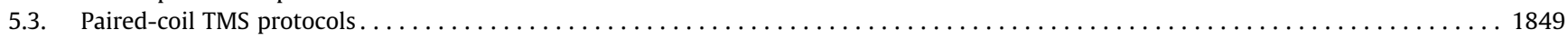

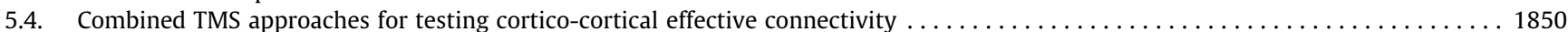

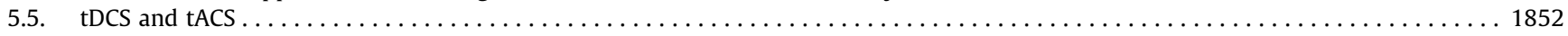

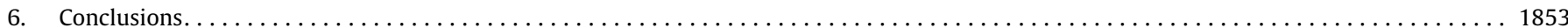

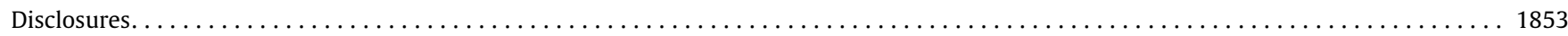

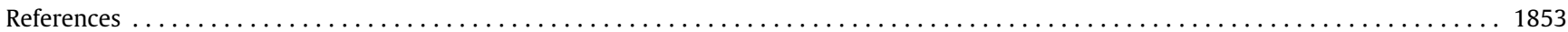

\section{Introduction}

The human brain contains about one hundred billion neurons, each establishing several thousand synaptic connections in an intricate matrix, which can be mathematically modeled in several ways. One approach models the brain as myriads of oscillators (i.e., cyclic firing of individual neurons and/or of spatially separated neuronal assemblies) organized in network structures at micromeso-macro-scale levels, with nodes and links that dynamically cooperate with time-varying aggregations via transient locking/ unlocking (i.e. orchestrated synchronization) of their cyclic firing (Singer, 1990; Jung et al., 2001; Makeig et al., 2002; Fuentemilla et al., 2006; Fries, 2015). Neural networks continuously re-shape via plastic mechanisms of synaptic Long Term Potentiation/ Depression reflecting the flow and type of input from internal and external environments, including daily experiences, learning/training, and emotional and aging processes. Therefore, when evaluating the brain's anatomical and functional organization from the perspective of complex networks (Bassett and Bullmore, 2006; Bullmore and Sporns, 2009; Sporns, 2011), the neuronal system can be modelled by a set of nodes (anatomical/functional neuronal aggregates) and interconnecting edges (structural/functional connections) (Fig. 1). This kind of architecture is regarded as a key substrate for two fundamental, coexisting and dynamically 


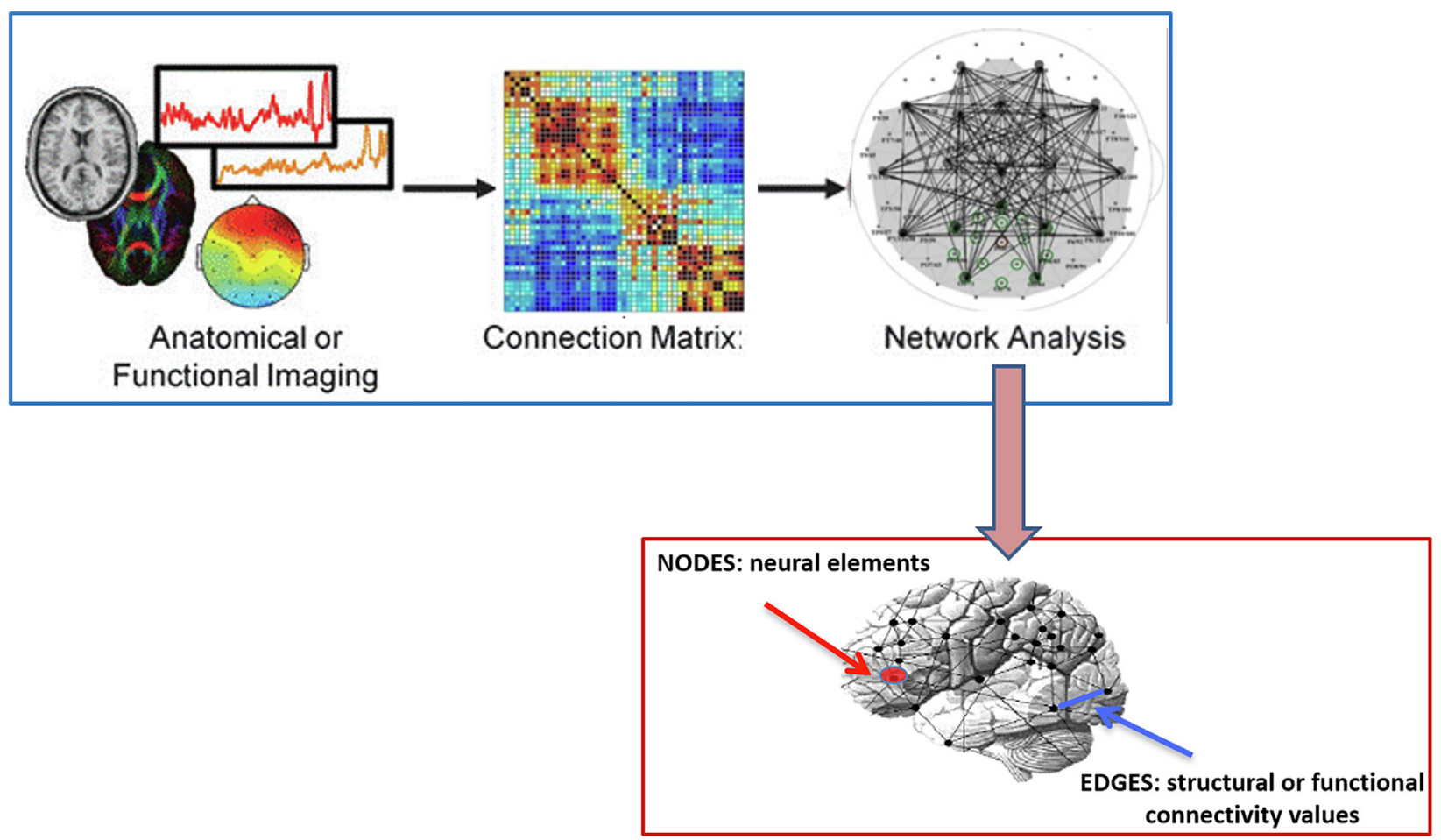

Fig. 1. Construction of a brain network.

interplaying brain properties: (1) the functional segregation of different regions and their involvement in cognition, sensorimotor integration, perception, and behavior (Tononi et al., 1994); (2) the functional integration ranging from the neuron (microscale) to inter-areal interactions (macroscale), to overall cognitive and behavioral output (Sporns and Zwi, 2004). In recent years, numerous studies approached human brain modeling using a new multidisciplinary method known as complex network analysis, with the aim of classifying neuronal networks with a small number of neurobiologically meaningful and easily computable measures (Rubinov and Sporns, 2010) and creating its connectome (Sporns, 2012). Modern brain mapping techniques - such as diffusion MRI, functional MRI, Non-Invasive Brain Stimulation (NIBS), EEG, and MEG- have produced and continue to produce increasingly larger datasets of anatomical or functional connection patterns. While neuroimaging techniques are able to faithfully reproduce the scaffold where the "quest" for brain function dynamics take place within a time frame during which inhibitory and facilitatory connections fluctuate simultaneously, such temporal dimensionsparticularly the time epochs which selectively define connectivity patterns before, during, and following a given task-can be reliably discerned by different brain mapping techniques. By explicitly defining anatomical and functional connections on the same map of brain regions, network analysis is a powerful tool for exploring structural-functional connectivity relationships (Zhou et al., 2006; Honey et al., 2007, 2009; ) and revealing the causative linkage between connectivity changes and task performance in the healthy or presence/severity of symptoms in neurologic neuropsychiatric disorders and aberrant connectivity (Stam et al., 2007; Bassett et al., 2008; Leistedt et al., 2009; Stam et al., 2009). The goal of this review is to examine existing and innovative methods for the human brain connectome exploration -particularly for the neurophysiological ones- providing measures of local and global connectivity from an IFCN-endorsed meeting of a panel of international experts. The present review complements another IFCNendorsed guideline on topographic and frequency analysis of resting-state EEG rhythms (Babiloni et al., 2018, in press).

\section{Structural brain connectivity: Experimental approaches and in vivo studies of the human brain}

\subsection{Chasing neuronal circuits: A never-ending story}

Over the centuries, many paradigm shifts have occurred in the views on neuronal connections, their behavioral output and their alterations in diseases (Bentivoglio and Mazzarello, 2010). The "neuron doctrine", which extended cell theory to the nervous system, was enunciated in 1891 (Shepherd, 2015). A breakthrough in the visualization of neurons was provided by the "black reaction", the metallic impregnation introduced in 1873 by Camillo Golgi (1843-1926). Golgi staining revealed neurons, including their processes, in their entirety and with unprecedented detail. This allowed studies of neuronal circuits (Golgi, 1885), and still allows the investigations of the local neuronal circuitry of randomly impregnated neurons (Fig. 2A), also in tissue blocks of postmortem human brain. The revelation power of the Golgi method is only matched after more than one century by genetic cell tagging with fluorescent proteins, or intracellular neuron filling (e.g., in surgically resected tissue blocks of the human brain) (Fig. 2B and C).

The champion of the "neuron doctrine" was Santiago Ramón y Cajal (1852-1934), who accomplished a monumental work, largely based on the Golgi stain, in which he provided a map of neuronal connectivity in the mammalian brain (Cajal, 1909, 1995). The debate between Cajal and Golgi-who had adhered to the reticular theory of nervous system organization-boosted neuroscience studies, focusing interest on the gray matter. White matter investigations were essentially descriptive, based on manual dissections and on the study of brain sections with the myelin stain introduced by Carl Weigert (1845-1904). Seminal contributions on the organization of fiber bundles in the human brain were provided by Carl Wernicke (1848-1900) and Joseph Jules Déjérine (1849-1917) (Schmahmann and Pandya, 2007).

The second half of the twentieth century witnessed a revolution in the experimental studies of neuronal connections, together with 

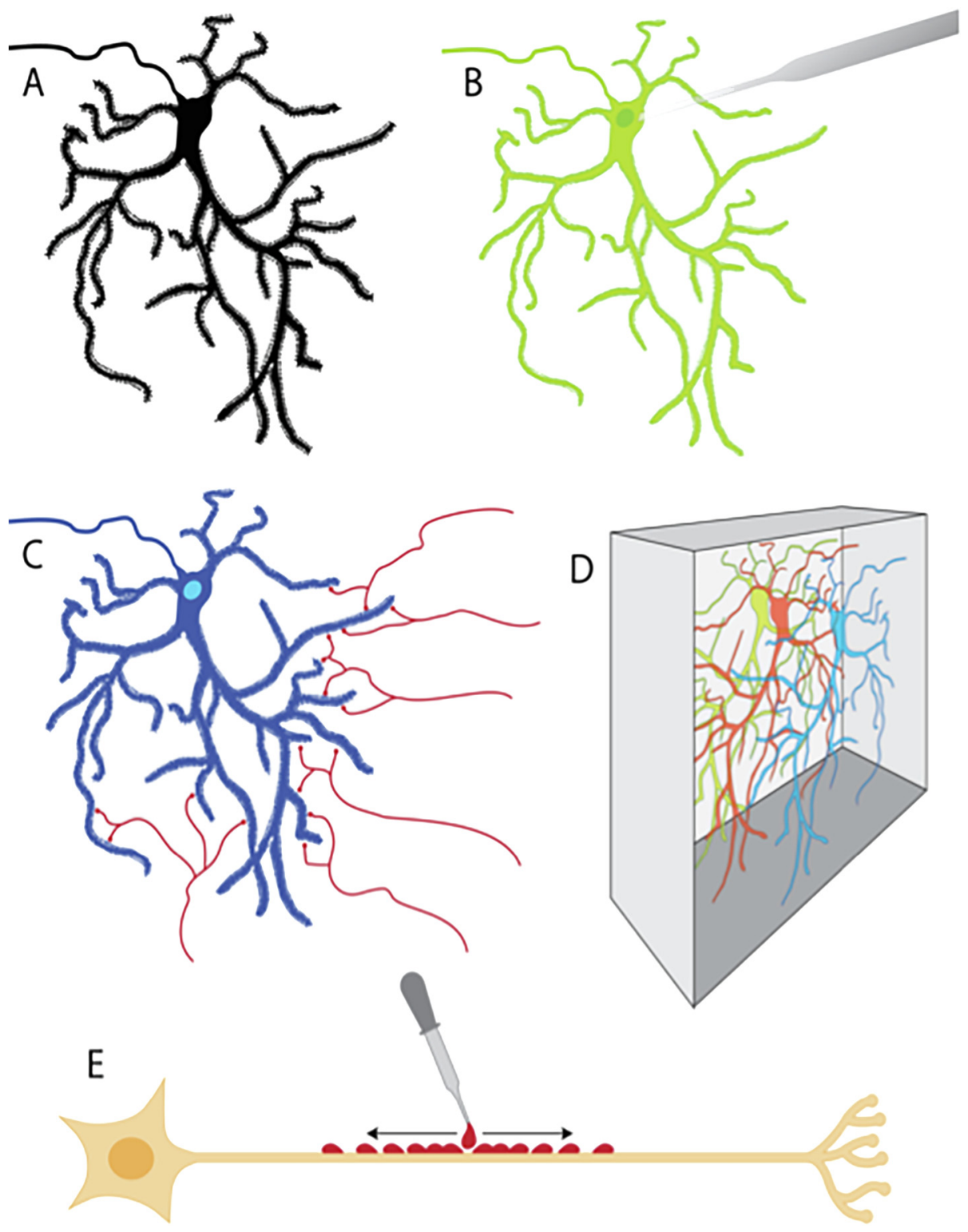

Fig. 2. Histological methods to study local neuronal connectivity and applicable to human brain samples. (A) The Golgi silver impregnation entirely fills neuronal cell bodies and their processes, allowing detailed visualization and reconstructions; on the other hand, with the Golgi stain it is impossible to predict which cells will be impregnated in any given preparation. (B) Filling neurons with fluorophores, as part of in-vitro electrophysiological experiments (for example in surgically resected brain tissue), allows correlating microscopic morphology with the functional properties of individual neurons. (C) Immunocytochemistry targets specific cellular markers, and combining different labels allows the study, for example, of connectivity at the individual synapse level. (D) Schematic representation of the clarification approach in which brain tissue blocks are rendered transparent and immunocytochemically labeled neurons can be visualized in 3D. (E) Lipophilic dyes applied on ex-vivo samples of nervous tissue are taken up by cell membranes and diffuse to a certain distance, thereby tracing short-range connections, also in human preparations.

the explosion of neuroscience in the last decades of the century. As briefly discussed below, novel powerful techniques were introduced. The exploration of connectivity in the human brain remained, however, a challenging problem until the introduction of in vivo imaging.

\subsection{Long-range neuronal connectivity}

\subsubsection{Anterograde and retrograde degeneration techniques}

Pioneering early studies revealed that retrograde degeneration ("secondary atrophy") of neuronal cell bodies and anterograde degeneration of fibers can provide effective tools to trace neuronal connections (Bentivoglio and Mazzarello, 2010) (Fig. 3A). Towards the end of the nineteenth century, neuronal alterations consequent to retrograde damage could be assessed by the cell stain (with thionin or toluidine blue) introduced in 1884 by Franz Nissl (1860-1919). Especially influential was the observation of anterograde degeneration of nerve fibers after transection reported in 1851 by Auguste Volney Waller (1816-1870) and named after him "Wallerian degeneration" (Fig. 3A).

Besides its implications for the trophic dependence of the axon from the cell body, this finding paved the way to the introduction of anterograde tract tracing methods based on silver impregnation of degenerating fibers after experimental lesions (Nauta and Gygax, 1951; Fink and Heimer, 1967). Metal impregnation stains are capricious and laborious, and degeneration methods have limited sensitivity, but these techniques gave a great impulse to experimental neuroanatomical studies. Importantly, anterograde degeneration revealed by modifications of silver impregnation was also applied to post-mortem investigations on the human 

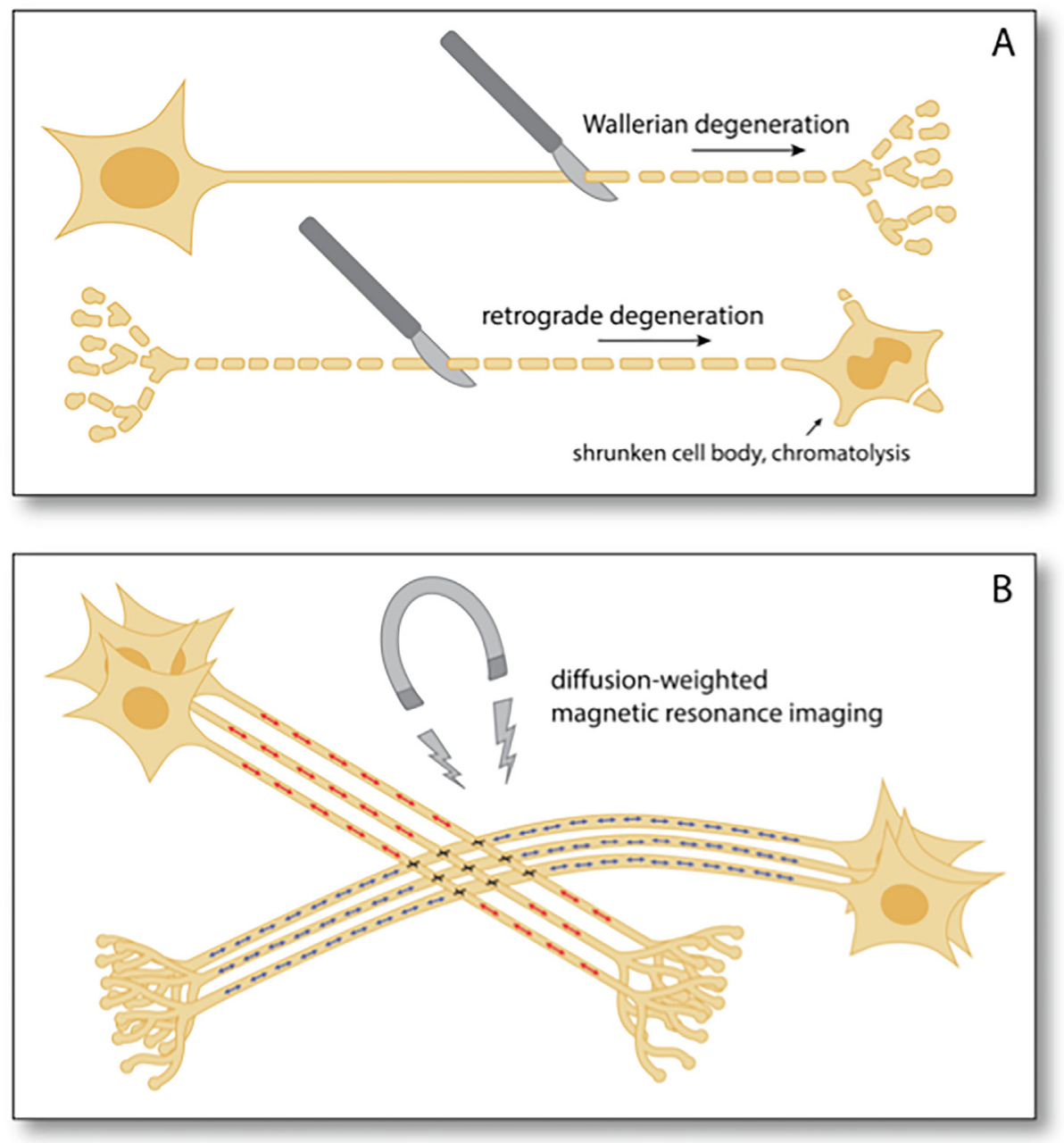

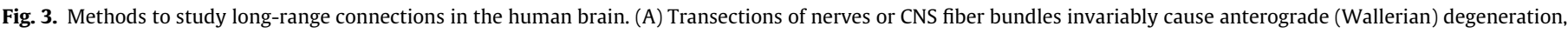

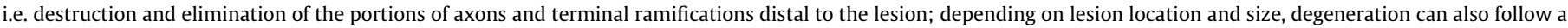

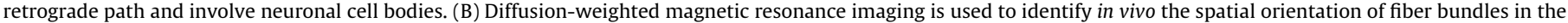

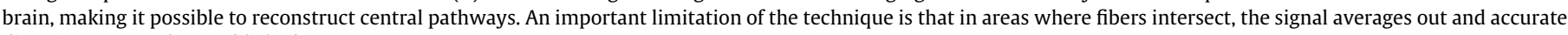
directions cannot be established.

brain, especially after restricted lesions occurring a few weeks before death (Mesulam, 1979).

\subsubsection{Classical experimental tract tracing techniques based on axonal transport}

A turning point in the study of structural brain connectivity was the discovery of anterograde and retrograde axonal transport (Bentivoglio, 1999). Axonal transport requires live axons; the active transport of tracers obviously cannot be applied to the human brain. Findings obtained with tract tracing based on axonal transport represent nowadays the "ground truth" for studies of the human brain based on in vivo imaging, and in particular on diffusion tractography.

Anterograde tract tracing based on the use of tritiated amino acids revealed by autoradiography was introduced in the early 1970s (Cowan et al., 1972). With this approach, trajectories and terminal fields of fibers originating from the tracer injection site could be delineated in detail. Anterograde tract tracing approaches have then been implemented (Gerfen and Sawchenko, 1984; Glover et al., 1986). In the same years, the discovery of retrograde axonal transport (Kristensson, 1970; Kristensson and Olsson, 1971) introduced as a tool the enzyme horseradish peroxidase (HRP), visualized by a histochemical reaction, which was soon applied to experimental retrograde tracing of the origin of projections to the tracer injection site (LaVail and LaVail, 1972).

The introduction of other retrograde tracers rapidly followed to increase sensitivity, combine tracers for multiple retrograde labeling for the study of branched connections, combine retrograde tracing with immunohistochemistry or in situ hybridization for the neurochemical characterization of pathways, and so forth. Fluorescent retrograde tracers turned out to be especially effective and versatile for these applications (e.g. Bentivoglio et al., 1980; Kuypers et al., 1980; Schmued and Fallon, 1986).

Conventional tract tracing has been implemented in recent years with genetic tracing for the study of the connectivity of specific neurons using cell-type-specific promoters (Oh et al., 2009). Most anterograde and retrograde tracers explore monosynaptic connections since they can cross synapses only in minute amounts, ineffective for transsynaptic tracing unless a bolus is injected, which is not feasible in the brain. Neurotropic viruses, which travel through axons and replicate in infected neurons, can instead provide tracing tools (Kristensson et al., 1974) applicable to trans-synaptic tract tracing (Kuypers and Ugolini, 1990) thanks to their propagation across synapses. 


\subsubsection{Novel approaches to experimental tract tracing: Optogenetics and chemogenetics}

These innovative techniques are increasingly used to investigate the relationship between neuronal activity, neuronal circuits, and behavior.

The term optogenetics was introduced in 2006 (Deisseroth et al., 2006) referring to the general optogenetic discovery (Boyden et al., 2005). By combining genetic and optical methods, optogenetics utilizes molecular light-sensors to switch on and off neuronal electrical activity. Optogenetics thus allow to investigate neurons and neuronal circuits underlying specific behaviors at the time scale of milliseconds. By this approach, functional effects of defined neuronal cell types can be controlled in living tissue and in freely moving animals (Deisseroth, 2015). Optogenetics has also been combined with functional MRI for the experimental study of cell-type-specific contributions to behavioral output together with a "whole brain read-out" at the millimeter scale (Lee et al., 2017). From the translational point of view, applications of optogenetics in humans for therapeutic purposes are currently envisaged. Clinical applications of the optogenetic system will require obvious implementation and cross-disciplinary know-how (Delbeke et al., 2017).

The term "chemogenetics" was used to describe experiments of site-specific functional group modifications for the analysis of DNA-protein interactions (Strobel, 1998). Currently, the term is used to indicate the processes by which "designer macromolecules" interact with previously unrecognized small molecules (Roth, 2016). Over the past two decades, chemogenetically engineered molecules (kinases, non-kinase enzymes, G proteincoupled receptors, ligand-gated ion channels) have been used experimentally for cell-specific targeting; these molecules modulate cell signaling, turning neuronal circuits on and off. Among chemogenetically engineered protein classes, the most commonly used are the so-called Designer Receptors Exclusively Activated by Designer Drugs (DREADDs) (Roth, 2016).

\subsection{Local neurocircuitry in the human brain}

\subsubsection{Diffusion of dyes}

An attempt to trace connections in the human brain using in vitro diffusion of wheat germ agglutinin conjugated with HRP gave very limited results (Haber, 1988). More interesting findings were obtained using the diffusion of lipophilic dyes along cell membranes in fixed tissue blocks (Fig. 2E). The fluorescent dyes carbocyanines, and in particular Dil and DiO (Honig and Hume, 1989) proved useful for this application. However, dye diffusion can label axons only for a few millimeters, requiring a tracing time of several weeks. Other dyes have been introduced (Heilingoetter and Jensen, 2016), and in particular NeuroVue dyes, which can trace axons for slightly longer distances and at faster diffusion rates than carbocyanines (Fritzsch et al., 2005). The limitations of ex vivo tracing, however, hamper its application for extensive fiber tracking in the human brain.

\subsubsection{Seeing through: Tissue clarification}

The natural 3D structure of cells - especially neurons and glial cells, which extend their ramifications in many directions requires volumetric imaging. The heterogeneous chemical composition of biological tissues (mostly water, proteins, and lipids) generates substantial scattering of the transmitted light, especially at the interface between aqueous protoplasm and membrane lipids, thereby hindering microscopic observation of histological sections beyond a certain thickness. Replacing lipids with a medium characterized by the same refractive index as proteins can effectively render tissues transparent while preserving the native molecular profile and tissue structure, allowing the microscopic observation of the microcircuitry of labeled (e.g., by immunohistochemistry or fluorescent protein tagging) elements.

Aqueous-based clearing techniques are currently widely used and are based on the reduction of light scattering by immersion in a high-refractive-index molecule solution. A breakthrough has been provided by a brain-hydrogel hybrid formed by the so-called CLARITY (Clear Lipid-exchanged Acrylamide-hybridized Rigid Imaging/immunostaining/in situ hybridization-compatible Tissue Hydrogel) (Chung et al., 2013). The clarification of thick tissue blocks, such as those useful for the study of the human brain (Fig. 2D) remains, however, a challenge. A method to adapt CLARITY to human brain samples with a thickness up to $8 \mathrm{~mm}$ has been recently proposed (Morawski et al., 2018). Of note, bridging historical and modern approaches to microcircuits, the Golgi (Golgi-Cox) stain is currently optimized for the use with CLARITY approaches, and could be useful for the study of microcircuitry and the comparison with microstructure MRI data (Kassem et al., 2017).

\subsection{Diffusion tractography}

Diffusion-weighted imaging (DWI), a computational reconstruction method of diffusion-weighted MR images (tractography), allows quantitative estimates in vivo of the organization of fiber bundles (tractograms). The characteristic color coding of reconstructed fiber bundles results in images attractive also to the public at large, thus making this approach a very popular insight in the human brain. This method is extensively presented in another part of our review.

The diffusion coefficient measures the ease of the translational motion of water in tissues. Main DWI acquisition schemes are provided by diffusion tensor imaging (DTI) (Fig. 3B), diffusion spectrum imaging (DSI), and high angular resolution diffusion imaging (HARDI). DTI utilizes a tensor model (a matrix of measured diffusion in three orthogonal planes) to characterize the water diffusion properties through myelinated nerve fibers (Basser et al., 1994). Fiber orientation profiles derive from the statistical profile of the displacement of water molecules at a voxel scale and fiber trajectories are inferred from adjacent similar diffusion profiles (Thomas et al., 2014). DSI adds to DTI the capability of resolving multiple directions in each voxel (Wedeen et al., 2005), thus improving also the tracking of intersecting fibers. HARDI improves the accuracy of tractography by using a large number of diffusion-encoding gradients with a reasonable scanning time.

After the first validation study in the macaque brain (Parker et al., 2002), a number of validation studies have been performed, with rather positive or more critical conclusions. For example, the comparison of DSI in the light of extensive autoradiographic tract tracing data on long association pathways in the monkey cerebral hemispheres was found to replicate main features of these fiber tracts (Schmahmann et al., 2007). This comparison proved useful and effective for major cortical fiber bundles (superior, middle and inferior longitudinal fasciculi, fronto-occipital fasciculus, uncinate and arcuate fasciculi, cingulum bundle) (Schmahmann et al., 2007). Another study, based on DWI approaches to the monkey brain, reached more critical conclusions on the potential for accurate fiber tracing (Thomas et al., 2014). The results of a recent "open international tractography challenge", tractograms produced by 20 research groups turned out to contain $90 \%$ of the ground truth bundles, but were also reported to "contain many more invalid than valid bundles" (Maier-Hein et al., 2017). These results encourage innovation.

\subsection{Macroscale of connections}

An exhaustive description of the intra- and inter-hemispheric anatomical bundles connecting different cortical and subcortical 
areas and nuclei cannot be done within the frame of this review. However, a rapid overview of the main connecting fiber bundles is provided in the following (see Fig. 4).

There are a number of short and long association tracts that provide intra-hemispheric communication within the cerebral cortex. The cingulum is an example of a long association tract. Commissural fibers provide communication between homologous regions of the hemispheres, the largest one being represented by the corpus callosum. Other commissures include the anterior commissure, which connects homologous cortical frontal lobe areas and the fornix, which is the efferent projection from the hippocampus. The posterior fibers (called the post-commissural fornix) of each side continue through the hypothalamus to the mammillary bodies; then to the anterior nuclei of thalamus, which project to the cingulate cortex. The anterior fibers (pre-commissural fornix) end at the septal nuclei and nucleus accumbens of each half of the brain. The internal capsule is a projection tract that contains many fibers carrying information between cortical and subcortical regions and the spinal column. It descends lateral to the head of the caudate nucleus and medial to the lentiform nucleus and thalamus. The corona radiata is where the internal capsule fans out superiorly. It is divided into several parts: the anterior limb contains frontopontine and corticothalamic fibers; the genu contains corticobulbar fibers; the posterior limb contains corticospinal and parieto-occipito-temporo-pontine fibers; and the retrolentiform/ sublentiform part includes the auditory and optic radiations. The internal capsule becomes the crus cerebri in the midbrain.

Cascades of short association fibers interconnect modalityspecific primary with secondary sensory association areas and these latter with multimodal sensory areas located at the borders. They may remain within the gray matter of the cortex or pass through the superficial white matter between neighboring cortical areas as $U$ fibers. Long association systems connect the modalityspecific association cortex and the multimodal areas in the occipital, temporal and parietal lobes with the premotor and prefrontal cortex. Short association fibers interconnect the prefrontal cortex, the premotor area and the motor cortex with the primary somatosensory cortex. Connections from multimodal association cortices and prefrontal cortex (PFC) to limbic structures pass via the cingulum to the medial temporal lobe; other fibers originating from association cortices reach limbic structures via the insula. Most association connections are reciprocal.

\section{Methods for connectomics}

A long-term goal of neuroscience is to develop models that integrate brain structure and function to predict human perception, cognition and behavior (Goldstone et al., 2015; Pestilli, 2015), but they often lack characterization at the level of the individual subject. Neuroimaging research has only begun to address this knowledge gap, and substantial work needs to be carried out before we can reliably study individuality and variation of brain networks (Finn et al., 2015; Laumann et al., 2015; Smith et al., 2015). Recent proposals have been made for the development of innovative technologies that can enable the study of the computational architecture of brain connections in individuals. The brain connectome is comprised of both grey matter (GM) regions representing neuronal units of information processing (the nodes, Fig. 5A), and white-matter tracts (WM), serving as structural communication pathways (the edges, Fig. 5B) (Bullmore and Sporns, 2009). To date, methods for precision measurement of brain networks have been developed, but not been fully optimized and agreed upon. To approach this issue, innovative technologies for the study of the computational architecture of brain connections in individuals and building precision models of brain connection patterns have been proposed.

DTI and fiber tractography allow investigators to measure the properties of the connectome in living human brains at the mesoand macro-scale ( $\mathrm{mm}$ to $\mathrm{cm}$ ), providing information about brain computational machinery that -because of experience and training- changes over minutes, hours, days, months and years (Sowell et al., 2003; Fields, 2008b; Westlye et al., 2009; Behrens and Sporns, 2012; Jbabdi et al., 2015). Recent technologies allow

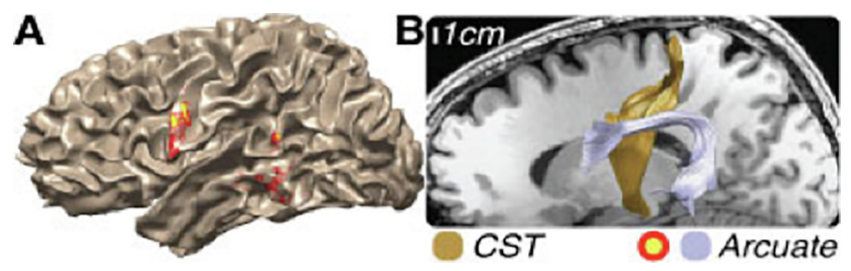

Fig. 5. (A) Connectome nodes. Cortical terminations of the arcuate fasciculus. Yellow higher, red lower termination density. (B) Connectome edges. Two major WM tracts, cortico-spinal tract (CST) \& arcuate fasciculus.

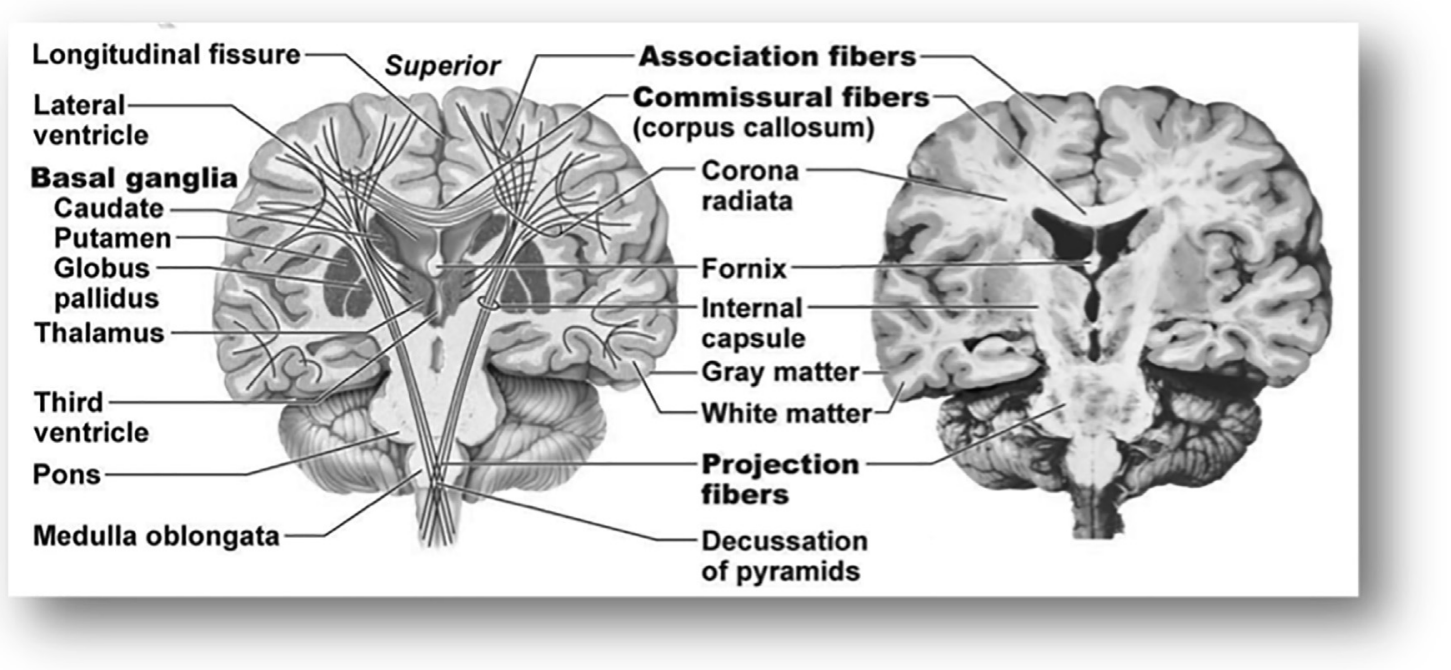

Fig. 4. Frontal section of the human brain (modified from $\odot$ Pearson Education, 2013) 
us to automatically identify major white matter tracts in living brains (see Fig. 5A and B) (Mori et al., 2005; Yeatman et al., 2012; Pestilli et al., 2014). These tracts are the most prominent edges in the connectome, information highways that implement communication about the senses, motor control, cognition and language. So far, we have learned that the biological properties of these tracts change transiently in response to experience, and can be steadily modified by learning, development and aging (Fields, 2008a, 2015; Risacher and Saykin, 2013). Even though much important work has been done to map the human connectome, to date the full set of connectome edges is still unknown, and we name only a few. Limitations to mapping the full human connectome have been due to two sources of variability in connectome estimates: (1) the dependency of connectomes on the tracking methods and (2) insufficient reliability of connectome estimates in individual brains or even in intra-individual brain when explored at different times. For example, Fig. 6A and B show eleven major human WM tracts identified in the same brain, using a single diffusion imaging data set, but two different tracking methods. These remarkable algorithm-dependent differences within single brains have imposed limits to the application of tractography for studying individuality and variation. Which algorithm should we use?

To approach these two issues, several methods have been developed. For instance, methods such as Linear Fascicle Evaluation (LiFE), have recently been proposed exploiting tractography evaluation. LiFE takes as input the set of white-matter fascicles generated using any tractography method and returns as output the subset of fascicles that predict the DTI measurements with smallest error. LiFE predicts diffusion measurements in individual brains by representing connectomes as systems of linear equations. It models the diffusion signal using the prediction of the combined WM fascicles in a connectome. Each fascicle is associated to a weight. Weights represent the contribution of fascicles to predicting the measured diffusion.

Fascicle predictions are organized by LiFE as columns of a large matrix of linear equations $(M)$. The diffusion signal $(Y)$ is predicted by least-square optimization $\|Y-M w\|_{2}^{2}$ (Eq. (1)). $M$ is a matrix where each column is a single fascicle prediction of diffusion and $w$ are the set of weights assigned to each fascicle to predict the diffusion measurements in all brain voxels $(Y)$. The root mean square error (r.m.s.) generated by the optimization (Eq. (1)) can be used to compare connectome models. Fig. 6C shows a comparison of r.m.s. error computed by LiFE for the two models in Fig. 6A and B. Errors were computed on a single brain from the Human Connectome Project (HCP) using LiFE and two different tractography methods: a probabilistic tractography method based on constrained- spherical deconvolution (Fig. 6A), and a deterministic tractography method based on the tensor model (Fig. 6B) using the HCP data (90 directions, $b$ value $=2000 \mathrm{~s} / \mathrm{mm}^{2}$ ).

Comparing connectome errors can be used to select the connectome models that best represent the diffusion measurements in a single individual. Better connectomes have lower error. The fundamental insight here is to use the r.m.s. error produced by LiFE to establish the accuracy of a connectome in a single individual. This error is proposed below as the foundation for developing a precision connectome science, a connectomic of the individual. Additional advances in methods for mapping structural connectomes have more recently exploited similar statistical evaluation approaches. Ensemble Tractography (ET; Takemura et al., 2016a), for example, uses a large set of candidate connections generated with multiple tractography algorithms to 'learn' the best connections, given the data; that best predict brain measurements. Fig. 6C shows that the probabilistic tractography model is better than the deterministic one in a majority of the voxels. Yet, the probabilistic model is not better in all voxels. In some voxels, the deterministic model does reasonably well. These are voxels where the r.m.s. error is below the diagonal. This indicates that the deterministic model is better in these few voxels. Thus, no single tracking method is always best - this insight motivated the development of a multiple tractography method, Ensemble tractography (ET). ET provides improvements over singletractography methods (STM). It is a new way of tracking that exploits ensemble methods. ET (1) identifies more WM connections, (2) increases white matter volume coverage, (3) decreases error in predicting the diffusion signal and (4) improves the anatomical representation of the human WM. All enhancements are achieved at standard data resolution. First, a set of single parameter connectomes (SPC) is created, each generated using a different tractography method (e.g., deterministic and probabilistic) or parameter setting (e.g., fascicle curvature). All these SPCs are combined into a single group. LiFE is used to find fascicle weights and eliminate all fascicles with zero weight. The result is the Ensemble Tractography Connectome (ETC) (Fig. 7).

ETCs consistently outperform SPCs. For example, the number of non-zero weight fascicles is higher for ETCs than any tested SPC. The proportion of total WM volume covered by the ETC is higher. The ETC is more accurate (lower error) than any tested SPC. Maps of the diffusion signal show better signal prediction by the ETC. Finally, ETCs contain important anatomical features absent in SPCs (Takemura et al., 2016b).

LiFE and Ensemble Tractography -or other modern methodscan be used to build customized connectomes for individual brains.
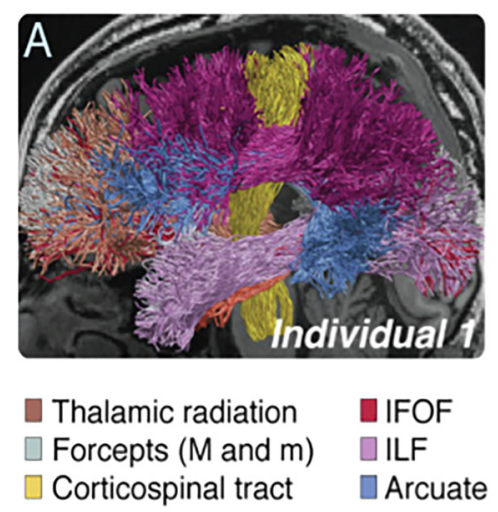

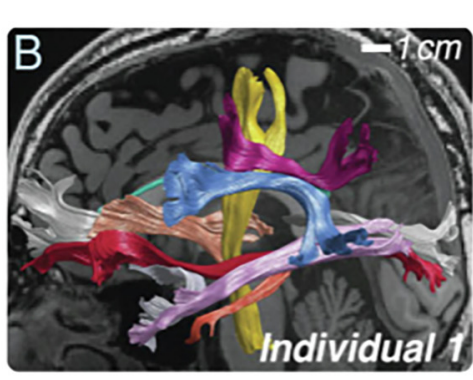

$\square$ Cingulum cingulate $\square \mathrm{SLF}$ - Cingulum Hippocam Uncinate

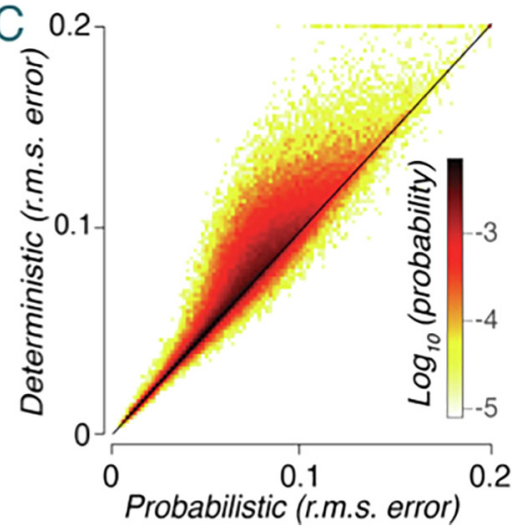

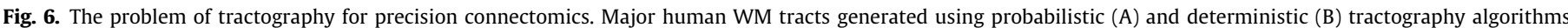

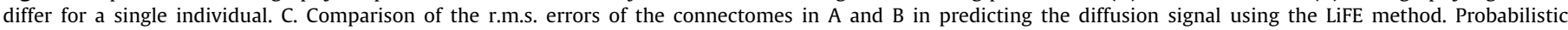
tractography (A) shows a smaller prediction error in a majority of voxels for this individual. 


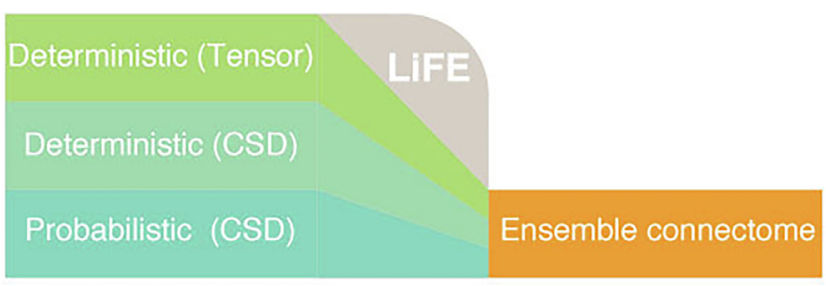

Fig. 7. Ensemble tractography (ET). Connectomes generated with multiple tracking algorithms are merged. LiFE is used to "learn" from the data. The ET connectomes contain all fascicles best contributing to predicting diffusion.

A crucial element necessary to apply the methods on many individual brains is the ability to run the methods routinely and efficiently. To tackle this challenge, preliminary results for a sparsefactorization method that drastically reduces the size of the LiFE model are briefly presented; this Sparse Factorized LiFE model $\left(\mathrm{LiFE}_{S F}\right)$ achieves accuracy quite close to the original LiFE, at a fraction of the computational cost. The factorization approach is an example of multivariate approaches that represent a new paradigm with the potential to open new avenues of investigation for computational neuroscience (McIntosh and Bratislav, 2013; Cichocki et al., 2015).

Pestilli and colleagues developed a sparse multiway factorization (Caiafa and Cichocki, 2013) to represent the LiFE matrix $M$ efficiently by combining a dictionary of precomputed diffusion prediction $(D)$ and an array $(\phi)$ of voxels, fibers and diffusion predictions in $D$ (Fig. 8A). The factorization approach compresses the model by eliminating redundancies and precomputing diffusion predictions. The approach can reduce the size of the LiFE model by factors of 30 or above; e.g., from $30 \mathrm{~GB}$ to $1 \mathrm{~GB}$ (Fig. 8B). $\mathrm{LiFE}_{S F}$ accurately approximates the original LiFE model (Fig. 8C). Preliminary results show that $\operatorname{LiFE}_{S F}(1)$ has r.m.s. error in predicting diffusion identical to LiFE (Fig. 6C scatter plot); (2) reproduces the LiFE matrix accurately (Fig. 6C top inset); (3) supports identical connectome weights as the original LiFE (Fig. 8C lower inset). The factorization method allows running the ET method routinely on many brains at scale, and create rich databases of candidate connections in individual brains that can be used to map variation in connections across large human populations.

Whereas previous approaches to brain connectivity (as described in the previous section) have focused on validating results using either animal models or synthetic data, a recent trend has been to use statistical approaches to evaluate results in individual brains, one brain at the time. These approaches focus on in-vivo brain measurements (for a review see Maier-Hein et al., 2017; Rokem et al., 2017; Wandell and Le, 2017). Recently, new approaches to evaluate and validate the results of tractography have been developed (Pestilli et al., 2014; Smith et al., 2014; Daducci et al., 2015; Takemura et al., 2016a; Caiafa and Pestilli, 2017). These approaches have the potential to advance discovery by providing mechanisms to evaluate and apply tractography to the study of individual brains, by leveraging statistical and computational methods (Pestilli, 2015; Wandell and Le, 2017).

\section{Techniques of measurement of brain function [fMRI, EEG, MEG]}

Perceptions and actions emerge from temporally coordinated local brain activities at multiple sites in distributed neuronal networks (Engel et al., 1991; Singer and Gray, 1995; Classen et al., 1998; Gerloff et al., 1998b; Singer, 1999a; Buzsaki and Draguhn, 2004; Engel et al., 2013; Bönstrup et al., 2014). This temporal coordination of brain activity can be measured at multiple scales and in distinct states of activation or rest with different metrics, each one with its own advantages and disadvantages. The interpretation of the measured signals is often challenging. In the human, EEG and MEG allow for non-invasive measurement of neuronal firing at high temporal resolution, i.e., in the range of milliseconds, however, with relatively low spatial resolution in the centimeter range. Excellent spatial resolution is in the domain of functional MRI (fMRI) which allows for the measurement of fluctuations of local blood flow and metabolism through detection of blood-oxygena tion-level-dependent (BOLD) changes of the magnetic field with millimeter precision. Another advantage of fMRI is its capability to detect activity changes in the depth of the brain much more reliably than EEG or MEG. However, its temporal resolution is limited due to physical properties of hemoglobin relaxation that introduces a significant time delay between the synchronized and relatively sharp neuronal firing and changes of local blood flow producing the BOLD signal with a smoothing effect on the firing sharpness during rise/decay times of the neurovascular reaction; moreover -and probably more important- the BOLD signal is based on changes of energy consumption and therefore it does not reflect those mechanisms of communication among neuronal assemblies which do not modify energy consumption (i.e. synchronization/coherence, phase locking-unlocking without any change of firing frequency/intensity). The spatial resolution of EEG and MEG is poor due to the relatively small number of channels and the non-uniqueness of the solution of the inverse problem; moreover such techniques are little or no sensitive at all to activity in neuronal assemblies located far from the scalp surface (either in the depth of sulci, or in the fronto-orbital and temporo-mesial areas including the hippocampal formation and insula) and/or due to a neuronal architecture producing a closed field organization like in subcortical nuclei. In the following survey (which has
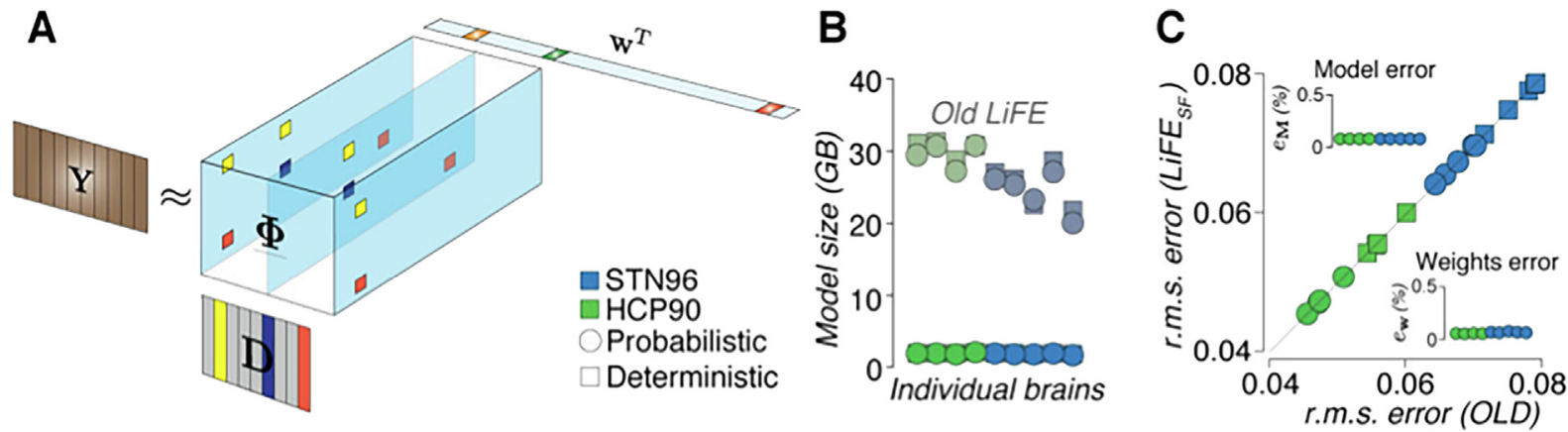

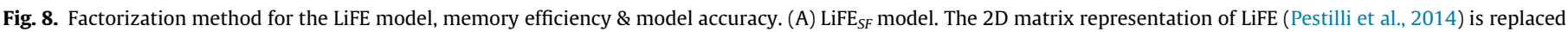

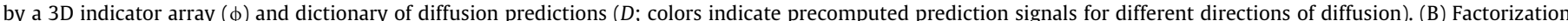

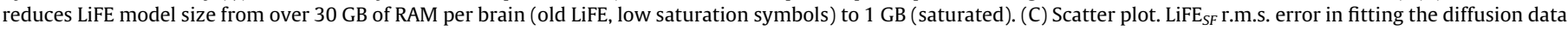
is indistinguishable from the original LiFE. Insets. Errors in reconstructing fascicles'weights and LiFE model are extremely low. 
a focus on electrophysiological methods), commonly used approaches and excerpts of their mathematical bases are described, some caveats are mentioned, and hints towards their interpretation are given.

\subsection{Resting-state vs. task-related measurements}

Historically, PET and fMRI allowed for describing some of the "scaffolds" of functional brain connectivity; they include the default mode, fronto-parietal, and dorsal attention networks. The brain's default mode network consists of discrete, bilateral and symmetrical cortical areas, in the medial and lateral parietal, medial prefrontal, and medial and lateral temporal cortices unexpectedly described in brain-imaging studies first performed with positron emission tomography in which various novel, attentiondemanding, and non-self-referential tasks were compared with quiet response either with eyes closed or with simple visual fixation (for a review see Raichle, 2015); the fronto-parietal network is mainly involved in task monitoring and reporting (for review see Koch et al., 2016); finally, the dorsal frontoparietal network assumes a puzzling variety of functions, including motor planning and imagery, mental rotation, spatial attention, and working memory (Ptak et al., 2017). In distributed networks, such as the described ones, neuronal activity at rest shows distinct spatiotemporal patterns of oscillatory fluctuations (for review see Engel et al., 2013). These so-called intrinsic coupling modes (ICMs) are of high interest as they contain significant information, request little or no changes of energy consumptions and have a meaningful spatiotemporal structure. It is likely that in the healthy brain ICMs reflect previous learning and can bias the processing of upcoming stimuli. In addition, any perception or action will impose some sort of (local or distant) synchronization, thereby altering the temporal relationship between signals. As a consequence, directly or stimulus-related (evoked) responses or oscillatory responses triggered by and outlasting the stimulus (induced) can be measured. All of them, ICMs and evoked or induced responses, can be described with several mathematical approaches at the network level. This type of neuronal coupling can be described in the time or the frequency domain.

In their pioneering work, Gevins and colleagues have used timeaveraged evoked potentials and their temporal covariation in order to describe simultaneous, connected EEG activity, so-called eventrelated covariances (Gevins et al., 1989). In more recent years, frequency-domain-based approaches have been more common in the analysis of human EEG and MEG data. The degrees of freedom of neuronal coding in the frequency domain are enhanced by the wide range of cyclic firing that can be generated by neuronal assemblies both in frequency (range between 0.025 and $600 \mathrm{~Hz}$; Bressler et al., 1993; Curio et al., 1994; Kandel and Buzsaki, 1997; Penttonen et al., 1999; von Stein and Sarnthein, 2000) and in amplitude. Coding in and across different frequencies of oscillation is of particular interest for EEG and MEG analyses. The ICMs are, at the very end of the very slow frequency spectrum (delta and sub-delta), mirrored to some extent by fMRI resting-state signals, which are increasingly used to reconstruct human brain networks (Cordes et al., 2002; Fransson, 2005; Scholvinck et al., 2010).

\subsection{Whole-brain approaches vs. hypothesis-driven analyses in subnets}

Coherence (Coh) (Gerloff et al., 1998b), partial coherence (pCoh), Phase-Locking Value (PLV) (Lachaux et al., 1999), Mutual Information (MI) (Kraskov et al., 2004), and Directed Transfer Function (DTF) (Kaminski et al., 1995; Bönstrup et al., 2014) are commonly used mathematical techniques to address interregional connectivity in EEG or MEG data. With respect to network nodes and connections, they are primarily hypothesis-free and can be used as whole-brain approaches. This is an advantage because it allows for an unbiased global view on resting-state or taskrelated changes in brain connectivity. However, this also carries some risk of false-positive results, or, if strictly corrected for multiple comparisons, can lack statistical power to detect modulations of subnets which are pivotal to a given function. An alternative is Dynamic Causal Modeling (DCM, Friston et al., 2003) where the modulation of interactions in preselected networks is analyzed. While initially applied to fMRI, this concept has also been extended to EEG/MEG (Kiebel et al., 2009). Of course, it is also possible to use the aforementioned methods like Coh, pCoh, PLV, or DTF hypothesis-driven on predefined networks with few nodes of interest. In contrast, DCM is not suited as an exploratory technique.

\subsection{Acquisition and processing of fMRI and EEG/MEG data for connectivity analyses}

\subsubsection{Functional MRI (fMRI)}

The use of functional MRI data relies on the blood-oxygenation dependent (BOLD) signal. The magnitude of the BOLD signal depends on multiple factors like, among others, the change in cerebral oxidative metabolic rate (CMRO2), the change in blood flow, volume, and oxygen extraction rate (Bandettini, 2014). Simultaneous measurements of BOLD signal and electrical neuronal activity indicate that the BOLD contrast reflects local field potentials (summed postsynaptic potentials) rather than spiking activity of neurons (Logothetis et al., 2001). MR scanners with 1.5 or 3 T field strength are typically used to acquire the raw data. In order to coregister the BOLD signals with individual anatomy, high-resolution T1-weighted anatomical images of the brain are measured as well (e.g., so-called MPRAGE). For functional imaging, gradient EPI sequences are used. To measure the BOLD signal, a subvolume is defined and, for example, one scan is acquired every 1-2 s. The spatial resolution of fMRI is high. Typical voxel size is, for example, $4 \times 4 \times 4 \mathrm{~mm}$ but even smaller voxels are possible. The exact settings vary depending on the experimental paradigm and the scanner used. In addition to block designs (task on/off) and event-related designs (time series of BOLD signal locked to single behavioral events), resting-state fMRI (rs-fMRI, Biswal et al., 1995) has gained much attention recently, especially with respect to connectivity analysis. It has become evident that voxel-wise correlations of BOLD signal time series contain information about the functional organization of the brain. For rs-fMRI, spontaneous fluctuations of the BOLD signal during rest are measured and analyzed to reconstruct neural networks (for review see, e.g., Keilholz et al., 2017). In general, fMRI data can be analyzed with various tools, e.g., with the Statistical Parametric Mapping software (Welcome Trust Centre for Neuroimaging, London, UK, http://www.fil.ion. ucl.ac.uk/spm), implemented in Matlab (The Mathworks Inc., Massachusetts, USA), or with BrainVoyager (Brain Innovation B. V., Maastricht, The Netherlands, https://brainvoyager.com). The strength of local activation in each voxel is typically calculated by means of multiple linear parametric modeling with general linear models (GLMs) of the measured BOLD signal, using a canonical synthetic hemodynamic response function (HRF).

\subsection{2. $E E G / M E G$}

EEG signals have been conventionally recorded from at least 19 scalp electrodes positioned according to the International 10-20 system, but the IFCN now suggests an extended array (see Seeck et al., 2017). The higher the number of simultaneously recording electrodes, the higher is the spatial resolution at least for sources on brain convexity (and the correspondence between the recording electrodes/sensors position and the functional relevance of the underlying cortical areas), but the higher the computational needs. The sampling rate of the EEG signal should be about four times the 
analog bandwidth; thus, it is sufficient to sample at $512 \mathrm{~Hz}$ when frequencies below about $130 \mathrm{~Hz}$ are investigated. If signals oscillating at $600 \mathrm{~Hz}$ are studied, a much wider bandwidth and higher sampling rate must be used (up to several $\mathrm{KHz}$ ). The monitoring of eye movements can be obtained with two different EOG channels, vertical and horizontal; skin/electrode impedances of all channels (this is an important issue only for EEG, but not for MEG) should be kept in the $\mathrm{k} \Omega$ range (preferably below $5 \mathrm{k} \Omega$ ) to minimize noise and external electromagnetic interference that may cause artifacts.

Before digitization (sampling) the EEG signals must be bandpass filtered so that the above-mentioned sampling-frequency rule is satisfied. Artifacts represent another important source of biased information. The digitized EEG data can be segmented to epochs (e.g., 2 s) for visually identifying and rejecting visible artifacts (i.e., eye movements, cardiac activity, and scalp muscle contraction); further, independent component analysis (ICA, a method widely used also in fMRI analysis) is very effective for further artifact rejection (Vecchio et al., 2017), but only provided that the artifacts are independent of the brain signals we wish to study. Thus, stimulus-triggered artifacts and stimulus-evoked brain signals are not generally fully separated by ICA, because they are not independent. Data can be analyzed with a number of different Matlab (MathWorks, Natick, MA) toolboxes, such as EEGLAB (Swartz Center for Computational Neurosciences http://www.sccn.ucsd.edu/ eeglab). ICA in EEGLAB can be performed using the Infomax ICA algorithm (Bell and Sejnowski, 1995).

While EEG is a powerful tool for measuring neuronal activity and connectivity, the lack of spatial resolution could be a drawback: as said before, in fact, EEG but also MEG are not sensitive to deep cortical activation. Inverse methods and approaches such as BEANFORM in MEG and LORETA in EEG data claim to detect deep sources but there is the possibility that a lot of information from deep structures in the higher frequency domains could be lost. Usually these methods allow to obtain good sources' reconstruction but it should be always taken considering their theoretical limitations.

\subsection{Metrics of connectivity in EEG, MEG, and fMRI}

\subsubsection{Coherence}

Coherence can be calculated for each frequency bin $\lambda$ according to the equation:

$$
\operatorname{Coh}_{x y}(\lambda)=\left|R_{x y}(\lambda)\right|^{2}=\frac{\left|f_{x y}(\lambda)\right|^{2}}{f_{x x}(\lambda) f_{y y}(\lambda)}
$$

which is an extension of Pearson's correlation coefficient $(R)$ to complex number pairs. In this equation, $f_{x x}$ and $f_{y y}$ are the auto-spectra, and $f_{x y}$ is the cross-spectrum of two signals $x$ and $y$ for a given frequency bin $\lambda$ (Gerloff et al., 1998b; Bönstrup et al., 2018). Two nodes A and B (generating the signals $x$ and $y$, respectively) can exhibit coherent activity because of emergent or stimulus/task/lesion-induced coupling of neuronal oscillatory activity but they can also be coherent because both nodes receive the same synchronizing input from a third node $C$ (generating the signal $z$ ). This could, in practical terms, be a higher-order cognitive area or a lesioned brain section controlling two connected brain regions in a top-down organization. Such scenario can be probed by applying partial coherence analysis which provides a measure of the coupling between two nodes, after taking into account any linear interaction between two signals $x$ and $y$ and a third signal $z$ which can be referred to as 'predictor'. Mathematically, the partial cross-spectra between $x$ and $y$, with $z$ as a predictor, are defined as:

$$
f_{x y / z}(\lambda)=f_{x y}(\lambda)-\frac{f_{x z}(\lambda) f_{y z}(\lambda)}{f_{z z}(\lambda)}
$$

where $\lambda$ is the frequency bin under study and $f$ denotes the spectral estimate of the EEG or MEG signals $x, y$, or $z$ for a given frequency bin $\lambda$. The partial auto-spectra of $x$, with $z$ being the predictor, are defined as:

$f_{x x / z}(\lambda)=f_{x x}(\lambda)-\frac{\left|f_{x z}(\lambda)\right|^{2}}{f_{z z}(\lambda)}$

The other partial auto-spectrum, $f_{y y / z}(\lambda)$, is defined likewise.

The actual partial coherence between the signals $z$ and $y$, with $z$ as a predictor, for the respective frequency bin $\lambda, \operatorname{pCoh}_{x y}(\lambda)$, can then be estimated similarly to the ordinary coherence as:

$\operatorname{pCoh}_{x y / z}(\lambda)=\left|R_{x y / z}(\lambda)\right|^{2}=\frac{\left|f_{x y / z}(\lambda)\right|^{2}}{f_{x x / z}(\lambda) f_{y y / z}(\lambda)}$

Coherence as well as partial coherence provides a measure of linear association, with values between 0 and 1 . For example, if $\mathrm{Coh}_{x y}$ is high but $\mathrm{pCoh}_{x y / z}$ (after removing the 'driving' influence of the third node $C$ ) is low, the interpretation would be that node $C$ contributes substantially to the coupling between nodes $A$ and B. If $\mathrm{Coh}_{x y}$ and $\mathrm{pCoh}_{x y / z}$ are similar in magnitude, then a relevant influence of node $C$ cannot be assumed. This is a relatively straightforward approach to get an impression about functional network interdependencies.

\subsubsection{Mutual information}

Mutual information (MI) is another metric of functional connectivity and similarly addresses the interdependence between two or more signals. However, in contrast to coherence, MI does not assume linearity of the interaction between the signals, i.e., it measures the linear and non-linear relationships (Kraskov et al., 2004). MI can be computed according to:

$$
I(X, Y)=\sum_{y \in Y} \sum_{x \in X} p(x, y) \log \left(\frac{p(x, y)}{p(x) p(y)}\right)
$$

where $\mathrm{p}(x, y)$ is the joint probability distribution function of the discrete variables $X$ and $Y$, and $\mathrm{p}(x)$ and $\mathrm{p}(y)$ are the marginal probability distributions of these variables (Kumar et al., 2017). Of note, functional connectivity measures like Coh, pCoh, or MI cannot differentiate between direct and indirect connections. It is always possible that two regions of interest, showing high coherence, are not connected at all but influenced by a third region. This needs to be taken into account when interpreting the results.

\subsubsection{Phase-locking value}

Coherence cannot reliably separate amplitude and phase contributions. The phase relationship between two signals independent of the amplitudes of the respective signals can be quantified by phase-locking statistics. To compute the PLV, bandpass-filtered epochs are Hilbert-transformed, then the phase $(\varphi)$ of the Hilbert-transformed data is extracted for all time bins $(t)$, trials $(n=1, \ldots, N)$, and all electrodes (EEG), sensors (MEG), or sources (EEG, MEG) if the calculations are done in the source space (e.g., after beamforming and spatial filtering). The difference of the phases between two electrodes $(\Delta \varphi)$ is then calculated at each time point $t$. The phase-locking value (PLV) is defined as the consistency of phase differences between two electrodes at a given time point $t$; it measures the inter-trial variability of the phase differences (Lachaux et al., 1999; Aydore et al., 2013), according to:

$$
\operatorname{PLV}_{t}=\left|\frac{1}{N} \sum_{n=1}^{N} \mathrm{e}^{j \Delta \varphi(t)}\right|
$$


where:

$\Delta \varphi(t)=\varphi_{m k}(t)-\varphi_{n k}(t)$

Here, $k$ indexes the trial number and $m$ and $n$ index the first and second channels of interest, always for a given time point $t$. The PLV separates amplitude and phase, and is thus less affected by amplitude variability of the power spectrum. PLV is less prone to be affected by volume conduction which represents one of the major confounding aspects for EEG (not for MEG) signal analysis.

\subsubsection{Directed Transfer Function}

From a physiological point of view, a matter of great interest is directionality, i.e., which node leads or lags in the interaction. One approach to address this is termed Directed Transfer Function (DTF) and requires multivariate autoregressive (MVAR) modeling of the epoched EEG (Kaminski et al., 1995) in the sense of Granger causality. In brief, every time point of each channel is predicted by the information that all the other channels' time series at previous time points offer. If knowledge of the past values of time series $\mathrm{X}$ significantly improves prediction of time series $\mathrm{Y}$, one can assume that there is a causal relationship between them. However, it has to be borne in mind that even if $\mathrm{X}$ does not influence $\mathrm{Y}$ but $\mathrm{Y}$ influences $X$, the measured past values of $X$ generally improve the prediction of $Y$, in particular if $X$ is measured with a higher signal-tonoise ratio than Y. Importantly, this relationship is not reciprocal and thus allows assessing the direction of information flow (Bönstrup et al., 2014). The time lag is predefined as the model order. The MVAR model of order $p$ can be described as:

$\vec{Y}(t)=\sum_{k=1}^{p} A(k) \vec{Y}(t-k)+\overrightarrow{X(t)}$

where $\vec{Y}(t)$ is the observed EEG data at channel $Y$ and time $t, \vec{X}(t)$ is the so-called innovation process, and $A(k)$ is the $k^{\text {th }}$ autoregressive parameter, with $p$ being the number of incorporated past time points. This gives a matrix of parameters $A_{x, y}(k)$ for each channel combination and time lag. This can be transformed from the time to the frequency domain in order to obtain derived measures in frequency space, which then can be described according to:

$\operatorname{DTF}_{x y}(f)=\frac{\left|H_{x y}(f)\right|^{2}}{\sum_{y=1}^{n}\left|H_{x y}(f)\right|^{2}}$

where $H_{x y}$ is the transfer matrix of the system. It contains information about all relations between the signals of interest (including phase relations). The $\mathrm{DTF}_{x y}$ describes directional influences of channel $x$ on channel $y$ at frequency $f$. This directionality of information flow can then be interpreted as causal influence of the 'sending' brain area on the 'receiving' brain area. Multivariate autoregressive statistics can also be combined with the concept of partial coherence, which is then referred to as partial directed coherence (see e.g., Huang et al., 2016).

\subsubsection{Dynamic causal modeling}

The techniques and metrics described up to this point have predominantly been applied to electrophysiological signals, less so to the slower modulations of the BOLD signal for fMRI (Arfanakis et al., 2000; Xiong et al., 1999). Network analyses based on the BOLD signal have been developed using multiple methods such as ICA (Independent component analysis) related methods (for reviews see: Bressler and Menon, 2010; van den Heuvel and Hulshoff Pol, 2010; Avena-Koenigsberger et al., 2017) Among these methods are the hypothesis-driven computation of interactions in pre-defined networks derived from anatomical or functional a priori knowledge, such as structural equation or dynamic causal modeling (Buchel and Friston, 1997, Penny et al., 2004).
Dynamic causal modeling (DCM) allows for addressing causal interactions between distinct (predefined) brain regions by constructing and testing realistic models of interacting neuronal areas (Friston et al., 2003). That is, away from a whole-brain approach and hypothesis-independent testing of possible interactions, DCM needs always to build on an extended a priori knowledge. DCM aims at estimating the coupling between brain areas and how that coupling is influenced by changes of the experimental context. Starting from a neuronal model of interacting cortical regions, DCM adds a forward model of how neuronal or synaptic activity is transformed into a signal that can be measured by fMRI (BOLD) or EEG (or MEG). For fMRI, this includes a hemodynamic response model to explain how neuronal electric activity translates into BOLD changes. Besides its use on fMRI data (Friston et al., 2003, 2011, 2014), DCM can also be applied to EEG and MEG data (David et al., 2006, Kiebel et al., 2006). The multimodal use of DCM for fMRI and DCM for induced responses in EEG/MEG (DCM-IR) is challenging but-when the intrinsic differences of the methods are carefully taken into account-it can generate mutually confirmative results that might be more robust and give deeper insights into cortical physiology than separately (Bönstrup et al., 2016). Technically, in a Bayesian framework, DCM models the instantaneous change of a neuronal state vector $z$ based on a neurodynamic forward model of how neuronal activity is transformed into the measured response. This can be described by:

$\frac{d z}{d t}=\left(A+\sum_{j=1}^{m} u_{j} B^{(j)}\right) z+C u$

where $t$ represents the continuous time and $u$ the $\left(j^{\text {th }}\right)$ experimental input. In DCM for fMRI, endogenous context-independent coupling among the different regions is described by the so-called A-matrix. Changes in coupling parameters that are caused by the experimental input (contextual modulators) are represented by the B-matrix. Finally, the C-matrix specifies which regions receive exogenous influences of inputs on neuronal activity. Parameters in the A, B and $\mathrm{C}$-matrices are estimated during the model inversion process and describe the architecture and interactions among brain regions at the neuronal level.

For DCM, the choice of a proper experimental design is crucial because this approach was designed for explicitly testing specific hypotheses rather than for using it as an exploratory tool. For example, a straightforward experiment for DCM analysis would be a sensory stimulation that is applied in multiple perturbated ways in combination with another factor that changes systematically the context of the sensory-evoked responses. As Friston pointed out in his original paper, the former could be a variation of visually presented words and the latter could be either the cognitive set or simply time (i.e., change of context by learning/plasticity over time) (Friston et al., 2003). More recently, however, also a DCM for resting-state data has been proposed (Friston et al., 2014).

The model of DCM-IR (for MEG/EEG) assumes that the interactions between two brain regions in the frequency domain can be linear (within-frequency coupling) or non-linear (cross-frequency coupling) (C.C. Chen et al., 2008). In this model, the neuronal state vector $z$ at region $i$ is represented by spectral densities over $k$ frequencies according to:

$z_{i}(f, t)=\left[\begin{array}{c}z_{i}\left(f_{1}, t\right) \\ \cdots \\ z_{i}\left(f_{k}, t\right)\end{array}\right]$

The event-related spectral signal changes are modeled as the response of distributed coupled electric sources to a spectral perturbation (Bönstrup et al., 2016). 
In summary, DCM refers to 'effective' (causal) connectivity, addressing directional interactions between brain areas. It necessitates strict hypothesis-driven experimental designs and circumscribed, predefined neuronal network structures. In contrast, coherence, partial coherence, phase-locking value, mutual information analyses, or any other form of correlation analyses in the time or frequency domain relate to 'functional' connectivity, i.e., metrics of neuronal coupling that do not allow for conclusions on causal interactions or direct versus indirect pathways between nodes of interest. Functional connectivity measures can be supplemented by information on directionality if combined with DTF or other forms of MVAR-based computations (e.g., partial directed coherence).

\subsection{Functional connectivity analysis with LORETA}

EEG recordings can be used for estimating the neuronal electrical activity distribution (current density vector field) on the cortex. Time series of cortical electrical neuronal activity can be analyzed, for example, with the minimum-norm estimation method (MNE; Hämäläinen and Ilmoniemi, 1994) or LORETA, to estimate cortical connectivity, based on the following informal definition: "Two places are functionally connected if their activity time series are similar" (Worsley et al., 2005). However, from a formal point of view, there are many different ways to define similarity between signals. Here, the "exact low resolution electromagnetic tomography" or eLORETA method is introduced (Pascual-Marqui et al., 2011). The eLORETA algorithm is a linear inverse solution for EEG signals that has no localization error to point sources under ideal (noise-free; error-free volume-conductor modeling) conditions (PascualMarqui, 2002). The connectivity values are obtained by Lagged Linear Coherence algorithm as a measure of functional physiological connectivity. Based on the scalp-recorded electric potential distribution, eLORETA computes the cortical three-dimensional distribution of current density (Pascual-Marqui, 2007a, 2009). Several recent studies (Canuet et al., 2011; Barry et al., 2014; Aoki et al., 2015; Vecchio et al., 2014a, 2014b; Ikeda et al., 2015; Ramyead et al., 2015; Vecchio et al., 2015, 2016) supported the idea of an accurate source localization using eLORETA. However, the expected accuracy can be realized only if the assumptions (e.g., source being sufficiently point-like) of the sources are valid. Via an individual analysis, brain connectivity is computed by eLORETA in the regions of interest (ROIs) defined according to the available Brodmann areas (BA) for left and right hemispheres (Talairach and Tournoux, 1988). Intracortical Lagged Linear Coherence, extracted by "all nearest voxels" or those in a sphere of 19-mm radius, selected on the basis of the number of considered nodes (Pascual-Marqui, 2007b; Pascual-Marqui et al., 2011), is individually computed between all possible pairs of ROIs for each EEG frequency band (Kubicki et al., 1979; Niedermeyer and da Silva, 2005): delta, theta, alpha 1 , alpha 2 , beta 1 , beta 2 and gamma. eLORETA current-density time series of each BA can be used to estimate functional connectivity; Lagged Linear Coherence (LagR) algorithm has been implemented in eLORETA as a measure of functional physiological connectivity not affected by volume conduction and low spatial resolution. For each EEG frequency, the mean connectivity matrix is computed between all frequency bins for each subject.

\subsection{Describing network properties by graph-theoretical parameters}

In order to describe properties of large, e.g., whole-brain networks the original empirical data can be represented in the form of a graph. Graph theory has been widely applied to MRI tractography (for a review see Crossley et al., 2016), but in this paragraph is mainly reviewed for applications in EEG/MEG analysis. This graph can be weighted or unweighted, and it can be directed or undirected. The first step is to decide what can be considered as a node, and what can be considered as a link (Stam, 2014; Miraglia et al., 2017).

Core measures of graph theory can be computed with http:// www.brain-connectivity-toolbox.net and adapted by Matlab scripts (Vecchio et al., 2014b; Miraglia et al., 2015, 2016). In such scripts, segregation refers to the degree to which network elements form separate clusters and correspond to clustering coefficient $(C)$ (Rubinov and Sporns, 2010), while integration refers to the capacity of the network to become interconnected and exchange information (Sporns, 2013); it is defined by the characteristic path length (L) coefficient (Rubinov and Sporns, 2010). The mean clustering coefficient is computed for all nodes of the graph and then averaged. It is a measure for the tendency of network elements to form local clusters (de Haan W et al., 2009). Starting by the definition of $L$, the weighted characteristic path length $L^{\mathrm{w}}$ represents the shortest weighted path length between two nodes (Onnela et al., 2005; Rubinov and Sporns, 2010). Small-worldness (SW) parameter is defined as the ratio between normalized $C$ and $L-C^{w}$ and $L^{w}$ - with respect to the frequency bands. For example, to obtain individual normalized measures, one can divide the values of the characteristic path length and of the clustering coefficient by the mean values obtained by the average values of each parameter in all EEG frequency bands of each subject. In this case, it should be stressed that a normalization of the data with respect to surrogate networks cannot be done due to the weighted values of the considered networks. The SW coefficient describes the balance between local connectedness and global integration of a network. SW organization is intermediate between that of random networks, the short overall path length which is associated with a low level of local clustering, and that of regular networks or lattices, and the high level of clustering which is accompanied by a long path length (Vecchio et al., 2014b). This means that nodes are linked through relatively few intermediate steps, and most nodes maintain few direct connections. Surrogate analysis plays a pivotal role for testing the significance of functional connections in both bivariate and multi-variate estimators; it also represents a significant methodological approach when applying a data-driven topological filtering scheme on statistically significant functional connections (Moharramipour et al., 2018).

Currently, network science is developing along a sophistication of network measures and models, introducing new concepts, such as cost-efficiency, hierarchical modularity, vulnerability to random or targeted attack, and the notion of rich clubs (as summarized in Fig. 9). An important challenge is to find simple yet meaningful ways to characterize brain networks while avoiding arbitrary choices and in addition to extract new diagnostic measures or biomarkers from network data (Stam, 2014).

Generally speaking, most of the studies on brain connectivity with various techniques are relatively weak because they do not report on inter- and/or intra-subject test-retest variability. In order to evaluate the within-subject test-retest variability (Vecchio et al., 2014a), statistical analysis was performed on normalized characteristic path length of EEG cortical sources for a 10 subjects group that accepted to come back for a second recording after about two weeks, introducing the factor Time (First and Second recording session). The statistical analyses showed no significant interaction including Time, highlighting the stability of the present methodology on "small world" analysis of EEG signal. More recently, findings from 3 recording sessions have been compared from 34 healthy subjects (mean age of 45 years) at one week distance one from the other. A between factors ANOVA was carried out: Frequency Band (delta, theta, alpha 1, alpha 2, beta 1, beta 2, and gamma) and Time (first, second and third recording) for the Small World parameter. The statistical analysis showed that the 


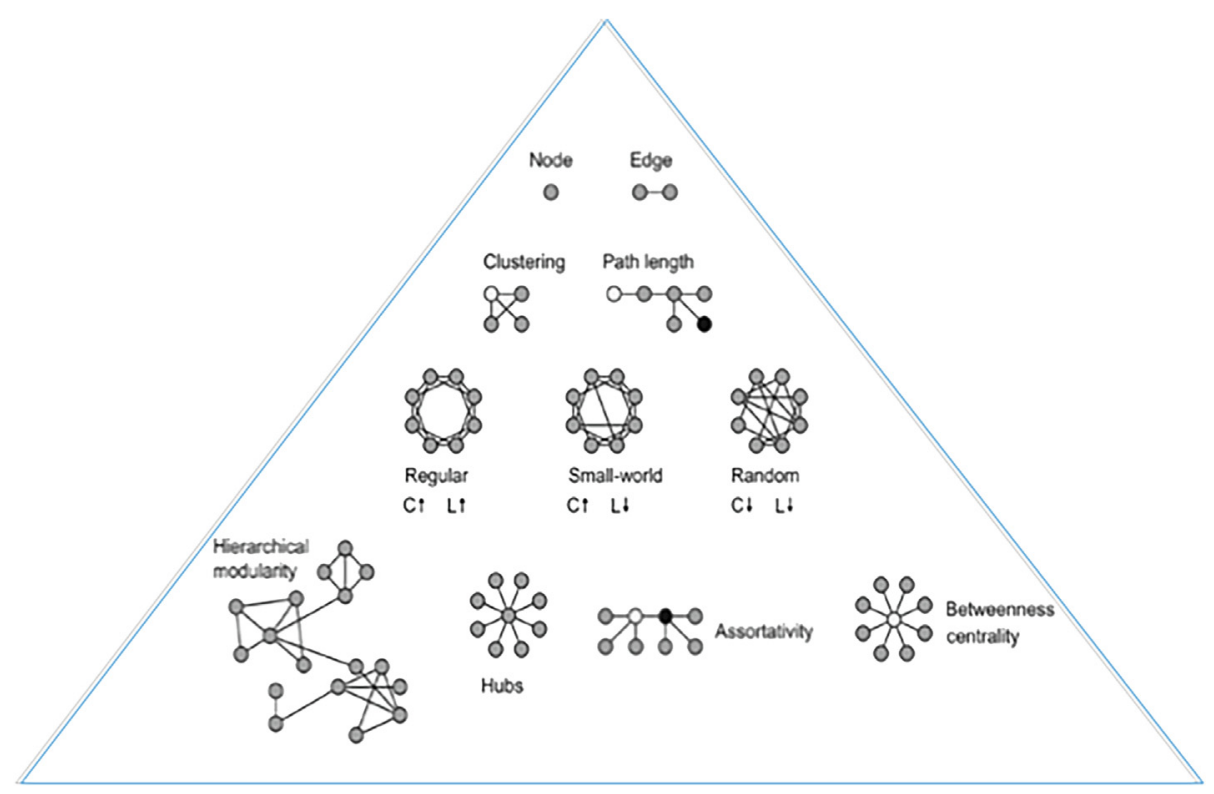

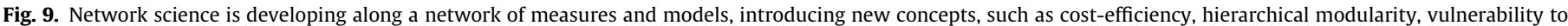
random or targeted attack, and the notion of rich clubs (from Vecchio et al., 2018).

interaction including Time was not significant $(\mathrm{F}(12,396)$ $=0.48995, p=.92057)$, highlighting the stability of the proposed parameters at least when carried out in clinically stable subjects. Recently, the importance of reliability studies based on repeatscan sessions protocol of connectomics in any modality has been recognized with publication of a number of freely available papers and datasets (Zuo and Xing, 2014; Colclough et al., 2016; Dimitriadis et al., 2017, 2018).

\subsection{The added value of multimodal connectivity analyses}

Non-invasive connectivity measures derived from EEG, MEG, or fMRI, but also from various (i.e., paired-pulse non-invasive brain stimulation) NIBS paradigms, naturally address 'only' certain aspects of the actual neuronal network activity. EEG and MEG depict population signals that closely resemble local field potential activity. fMRI measures a very indirect signal, which is composed of oxygen consumption and blood-flow changes as a reflection of transient modifications of energy consumption following similar changes in local neuronal firing. TMS probes responses elicited by non-physiological activation of the stimulated neuronal populations. In all of these studies, anatomical constraints to network structures should be taken into account. There are multiple ways to get around spurious results and wrong conclusions, starting with basic precautions like sufficient sample sizes and wellcontrolled experimental variables. However, this cannot exclude that the method selected has an inherent bias. For example, interregional inhibitory interactions are readily detected by TMS techniques (Gerloff et al., 1998a; Wahl et al., 2007), can regularly be modeled by DCM for fMRI (Rehme et al., 2011), but are less easily seen in EEG metrics (Bönstrup et al., 2016). Any network dynamics of neuronal firing that occur at the millisecond time scale which are separate, but adjacent in time will entirely or partly escape blood-flow related measurements like fMRI (or positron emission tomography or near-infrared spectroscopy) due to the time delay between neuronal firing and BOLD signal production and it smoothed rising/decaying slope unless they cause a secondary, sustained net effect on neuronal activity over longer periods of time (seconds to minutes at least); because of this, the timehierarchy of sources (nodes) connected in a network supporting a given brain activity cannot be easily discriminated by flow-metabolic techniques when the internode activation intervals are too short. Similarly, network dynamics which do not require changes in energy consumption (i.e. phase locking-unlocking with a stable firing frequency) do not produce a BOLD signal in fMRI. In fact, 'coding by synchrony' is possible in the absence of changes in energy consumption and of significant net changes of averaged neuronal population activity over time (Singer, 1999b). This implies that selecting a priori networks on the basis of significantly enhanced local activation may miss relevant network nodes. EEG and MEG (or invasive electrophysiological methods with millisecond resolution) better address this type of information coding in neuronal networks. On the other hand, neurophysiological techniques has a well-known limitation, that is the lack of information about locations of the brain sources: coupling between scalp EEG signals do not necessarily imply coupling between the underlying neural sources of EEG. In fact, scalp EEG recordings reveal not only averaged post-synaptic activity from localized cortical areas but also the overlapping activity of all coherent neural sources situated anywhere in the brain, together with the signal mixing owing to the volume conductance and reference electrode: this makes the interpretation of the sensor-space synchronization measures and evaluation pf large-scale connectivity difficult. Moreover, as repeatedly mentioned before, EEG and MEG are blind to most of the subcortical neuronal activity, including subcortical-cortical connections and those brain relays which are of paramount importance both in healthy and in diseased conditions like the hippocampal formation, the temporo-mesial region and the limbic areas. To minimize the bias inherent in each technique before drawing extensive conclusions on the physiology of neuronal networks, it may therefore be advisable to integrate two or more techniques -by carefully considering what they really reflect and do not reflect in brain function- and attempt to arrive at interpretations that hold true independent of the network-probing technique used. Mutually informative data have been derived from combinations of EEG and fMRI (Bönstrup et al., 2016), MEG and fMRI (Ahlfors et al., 1999), TMS and fMRI (Volz et al., 2014) but also from TMS and structural MRI metrics like DTI (Wahl et al., 2007, 2016) and many others (Nguyen et al., 2014; Klamer et al., 2015; Petro et al., 2017). With respect to modeling of neuronal networks, e.g., coupled oscillator models, informing functional connectivity matrices derived from EEG recordings integrated by structural 
information from DTI appears to be suitable and improves the model quality (Finger et al., 2016).

\section{Non-invasive brain stimulation (NIBS) methods for testing brain connectivity}

\subsection{MEP, I-waves}

The motor evoked potential (MEP) is recorded from a target muscle by surface electromyography (EMG) and reflects the activation of corticospinal cells in primary motor cortex (M1) by singlepulse transcranial magnetic stimulation (spTMS) (Barker et al., 1985). Therefore, the MEP is a marker of the connectivity between motor cortex, the alpha spinal motoneurons and muscle. The MEP amplitude increases sigmoidally with stimulation intensity (Hess et al., 1987; Devanne et al., 1997). Voluntary target muscle activation shifts this input-output curve to the left (Hess et al., 1987; Devanne et al., 1997; R. Chen et al., 2008). It is important to note that spTMS typically results in multiple descending corticospinal volleys, an early D-wave (for direct activation) followed by Iwaves (for indirect, i.e., synaptic activation) (Amassian et al., 1987). The neuronal mechanisms underlying the different Iwaves are still unclear (Ziemann and Rothwell, 2000; Triesch et al., 2015). Several hypotheses are being discussed, ranging from oscillating properties of the corticospinal cells to distinct circuits of excitatory and inhibitory interneurons impacting on corticospinal target cells (Kernell and Chien-Ping, 1967; Sakai et al., 1997; Di Lazzaro et al., 1998a).

The multiple descending, stimulus-triggered volleys are spatially and temporally integrated at spinal alpha motoneurons. If the summed excitatory post-synaptic evoked potentials reach the firing threshold, an action potential is generated that leads to excitation of the corresponding motor unit. TMS at low intensity activates primarily small motor units that are also first recruited with voluntary activation according with Henneman's size principle (Rossini et al., 1995). In summary, MEP measurements assess connectivity between a pathway consisting of cortical interneurons, cortico-motoneuronal neurons originating from layer $\mathrm{V}$ of $\mathrm{M} 1$, and alpha motoneurons in spinal cord and muscle (Lemon, 2008). Excitation of cortical interneurons and cortico-motoneurons is controlled by inhibitory interneurons (Ilic et al., 2002; Kawaguchi and Kondo, 2002; Markram et al., 2004). Therefore, MEP amplitude is influenced by the balance of excitation and inhibition in M1, which can be highly abnormal in neurological diseases and affected by CNS-active drugs (Ziemann et al., 2015). Also, the instantaneous state of M1 excitability impacts MEP amplitude, as has been revealed by recent measurements that combined EEG

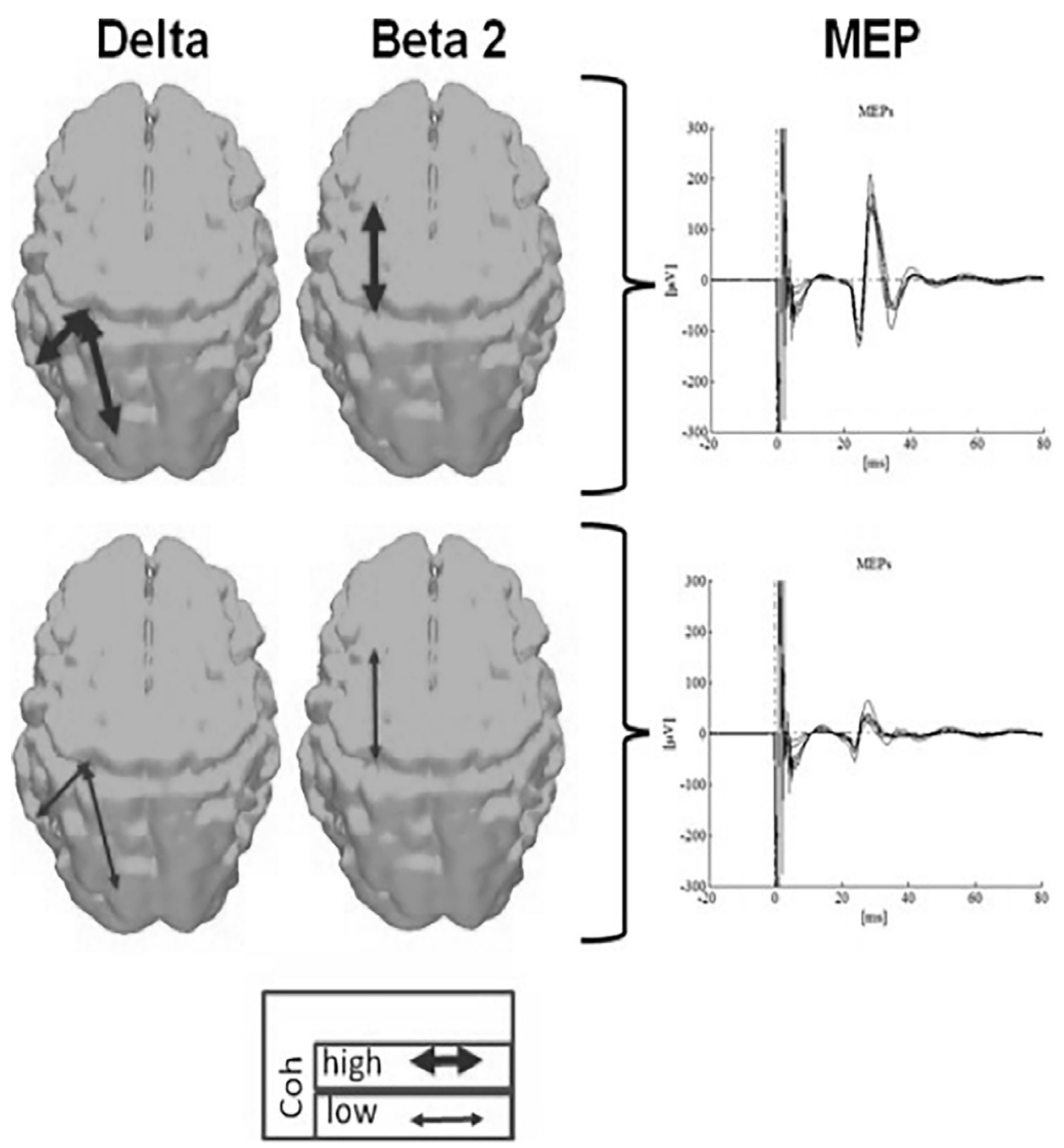

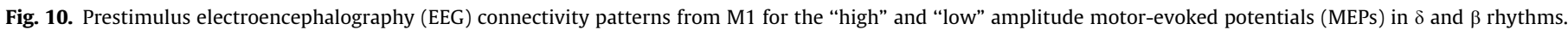

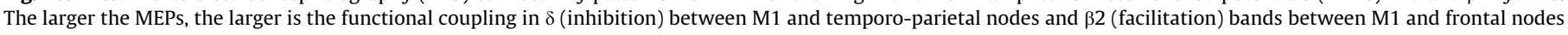
(from Ferreri et al., 2014; Vecchio et al., 2018). 
recordings of endogenous brain oscillations and the underlying connectivity networks with spTMS, as reported in previous papers (Mäki and Ilmoniemi, 2010; Bergmann et al., 2012; Keil et al., 2014; Ferreri et al., 2014; Giambattistelli et al., 2014; Triesch et al., 2015; Zrenner et al., 2018), and summarized in Fig. 10 (Ferreri et al., 2014); moreover this impact is age dependent (Ferreri et al., 2017a).

\subsection{Paired-pulse TMS protocols}

The paired-pulse TMS (ppTMS) protocols summarized here (Fig. 11) are defined by two pulses delivered through the same stimulating coil. These methods can be used to look at connectivity between neurons in a cortical column or in close neighboring brain areas. Stimulating over the motor cortex, if the first (conditioning) pulse is subthreshold for M1 excitation, the second (test) pulse suprathreshold, and if the interstimulus interval (ISI) is $1-5 \mathrm{~ms}$, then the test MEP is inhibited, (short-interval intracortical inhibition; SICI Kujirai et al., 1993). SICI can be elicited by first pulses of very low intensity, indicating that, according to a "cortical size principle", small inhibitory interneurons are activated and mediate this effect (Kujirai et al., 1993; Ziemann et al., 1996; Ilic et al., 2002). This is supported -at the 'micro' connectivity level- by pharmacological studies that reported increase of SICI by benzodiazepines, i.e., positive allosteric modulators at $\mathrm{GABA}_{\mathrm{A}}$ receptors (Ziemann et al., 2015). However, zolpidem with selective affinity to the alpha-1-subunit-bearing subtype of the $\mathrm{GABA}_{\mathrm{A}}$ receptor, did not enhance SICI (Di Lazzaro et al., 2007), strongly suggesting that SICI is largely mediated by the alpha-2-subunit-bearing subtype of the $G_{A B A}$ receptor and, therefore, by Chandelier cells that make predominantly synaptic contacts with the pyramidal cells axonal hillock (Kawaguchi and Kondo, 2002).

Experiments with two coils on top of each other demonstrated that SICI is local, i.e., it rapidly declined when moving the stimulating coil delivering the first stimulus away from the motor hot spot (Ziemann et al., 1996). Furthermore, SICI was independent of the orientation of the coil delivering the first stimulus, suggesting that the activated inhibitory interneurons do not have specific excitability characteristics linked to a particular stimulus orientation (Ziemann et al., 1996). Altogether, a bulk of scientific findings support -including epidural recordings- the concept that SICI is a marker of effective connectivity between local inhibitory interneurons and cortico-motoneuronal cells, and/or the excitatory pyramidal cells projecting onto cortico-motoneuronal cells (Di Lazzaro et al., 1998b; Ilic et al., 2002).

A ppTMS protocol with longer ISIs of 7-15 ms typically results in MEP facilitation, referred to as intracortical facilitation (ICF) (Kujirai et al., 1993; Ziemann et al., 1996) which is supposed to be a marker of effective connectivity between excitatory interneurons and cortico-motoneuronal cells.

A ppTMS protocol that uses a suprathreshold first and a subthreshold second stimulus (Ziemann et al., 1998), or two threshold stimuli (Tokimura et al., 1996) at short ISIs of 0.5-4.5 ms results in MEP facilitation at discrete ISIs of 1.1-1.5 ms, 2.3-2.9 ms and 4.1$4.5 \mathrm{~ms}$, with no significant facilitation in between (Ziemann et al., 1998). This is referred to as short-interval intracortical facilitation (SICF) with 3 peaks having an inter-peak interval of approximately $1.5 \mathrm{~ms}$ (equivalent to $660 \mathrm{~Hz}$ ) reminiscent of the interval separating two successive I-waves (see above Ziemann and Rothwell, 2000, Ziemann et al., 1998).

A ppTMS protocol that uses two suprathreshold pulses at ISIs of 50-150 ms results in MEP inhibition and is referred to as longinterval intracortical inhibition (LICI) (Valls-Sole et al., 1992). LICI is enhanced by baclofen, a selective agonist at the $\mathrm{GABA}_{\mathrm{B}}$ receptor (McDonnell et al., 2006); moreover, LICI duration is compatible with $\mathrm{GABA}_{\mathrm{B}}$ receptor-mediated inhibitory post-synaptic potentials (Connors et al., 1988). Evidence strongly suggests that LICI is a marker of effective connectivity between inhibitory interneurons and cortico-motoneuronal cells and/or the excitatory pyramidal cells projecting onto cortico-motoneuronal cells, through the $\mathrm{GABA}_{\mathrm{B}}$ receptor (Di Lazzaro et al., 2002).

The same ppTMS protocol of two suprathreshold pulses at ISIs of around 200-250 ms results in MEP facilitation, termed late cortical disinhibition (LCD) (Cash et al., 2010, Cash et al., 2011). The

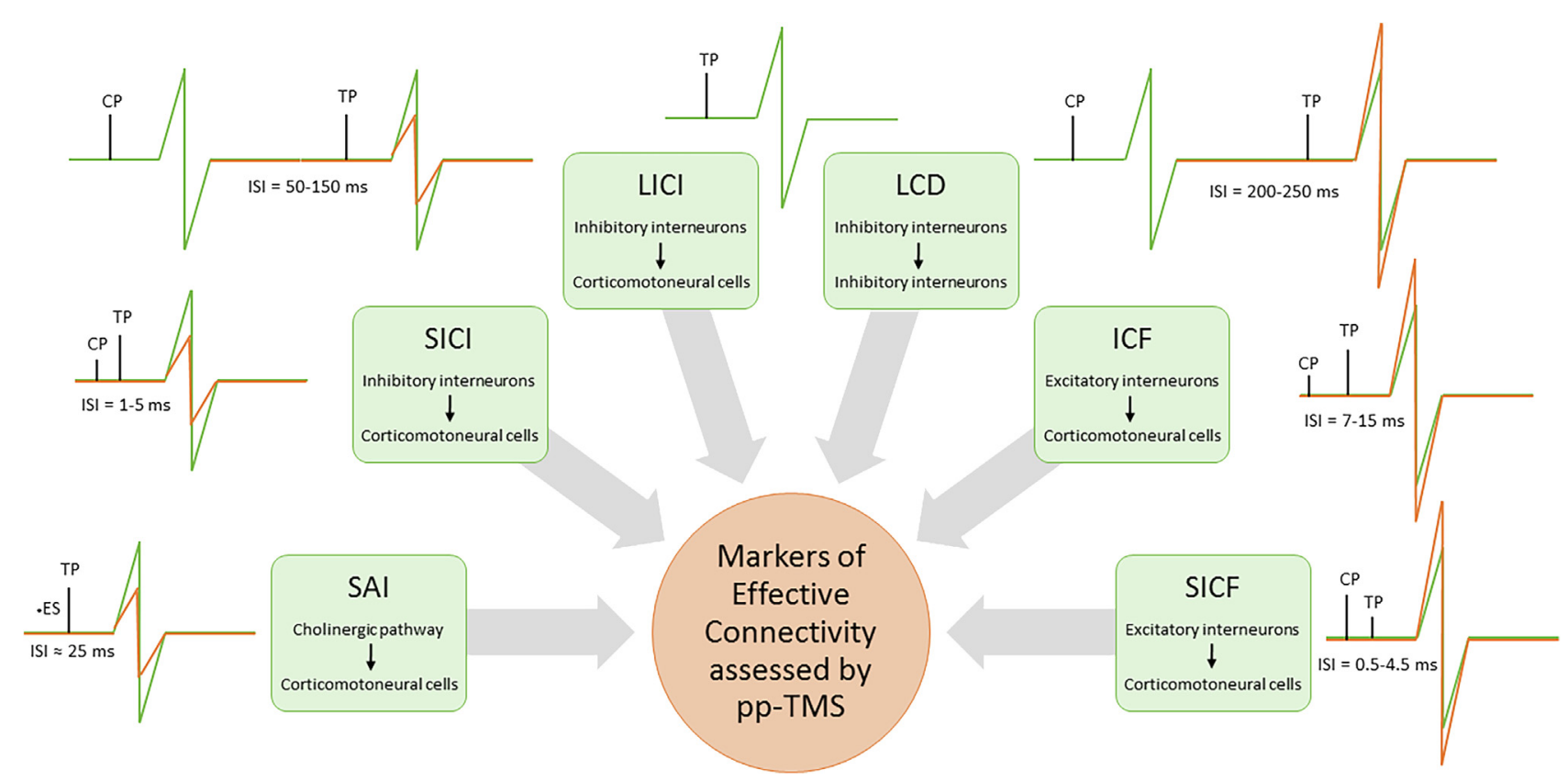

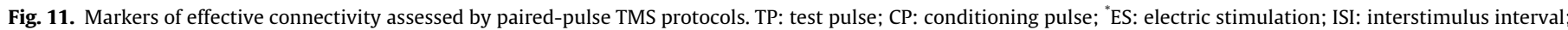

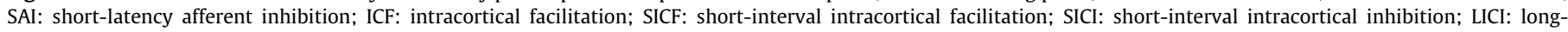
interval intra-cortical inhibition; LCD: late cortical disinhibition. 
mechanism underlying $L C D$ is likely $G_{A B A}$ receptor mediated presynaptic autoinhibition of $\mathrm{GABA}_{A}$ ergic inhibitory interneurons, as has been demonstrated in paired-pulse depression experiments (Deisz, 1999). Importantly, it was also shown that the duration of $\mathrm{GABA}_{\mathrm{B}}$ receptor mediated inhibitory post-synaptic potentials is shorter than the pre-synaptic autoinhibition (Deisz, 1999). In sum-

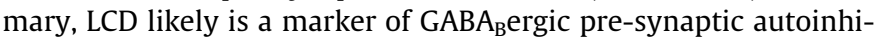
bition of inhibitory interneurons in M1.

Finally, peripheral nerve electrical stimulation (e.g., of the median nerve at the wrist) followed by suprathreshold spTMS of contralateral M1 provokes a MEP inhibition at ISIs of the individual N20 wave latency of the median nerve somatosensory evoked potential plus 0-4 ms while MEP facilitation is observed with ISIs of N20 wave latency plus 6-10 ms (Mariorenzi et al., 1991; Tokimura et al., 2000). The MEP inhibition is referred to as shortlatency afferent inhibition (SAI), and is associated with reduction of late I-waves but not the I1-wave in epidural spinal recordings (Tokimura et al., 2000).

\subsection{Paired-coil TMS protocols}

Paired-coil TMS (pcTMS) protocols deliver TMS pulses through separate coils to different sites of the brain and enable investigation of effective connectivity/mutual influence between separate brain areas; M1 and other, typically motor-related, areas of the brain, such as the contralateral M1, other frontal or parietal areas, and the cerebellum have been investigated by this technique (Hallett et al., 2017). In the pcTMS protocols, one stimulating coil is placed over the target M1 to elicit the test MEPs, and another one over a different area to test the effect of conditioning pulses on test MEP amplitude. Key parameters are timing and intensity of the conditioning stimulus (CS), in addition to placement of the conditioning TMS coil. Test stimulus (TS) intensity can also have an impact on the results.

A suprathreshold CS delivered to the M1 on one side given at ISI of 6-30 ms prior to TS of the contralateral M1 results in inhibition of the test MEP (Ferbert et al., 1992). Maximum inhibition occurs at an ISI of around $10 \mathrm{~ms}$, later termed short-latency interhemispheric inhibition (SIHI), which is mediated by transcallosal fibers projecting onto inhibitory interneurons in the test M1(Ferbert et al., 1992; Daskalakis et al., 2002; Kukaswadia et al., 2005; Müller-Dahlhaus et al., 2008; Ni et al., 2009; Tsutsumi et al., 2012). In addition to SIHI, other interhemispheric interactions have been reported, in particular, long-latency interhemispheric inhibition (LIHI) and interhemispheric facilitation (IHF). Except for the ISI, which is typically around 40-50 ms (Gerloff et al., 1998a), LIHI can be tested in the same way as SIHI. LIHI is believed to be mediated by a different mechanism compared to SIHI. Although both seem to be associated with $G_{A B A}$ receptor mediated inhibition (Daskalakis et al., 2002; Kukaswadia et al., 2005), only LIHI is related to the ipsilateral silent period, another spTMS measure of interhemispheric inhibition (Chen et al., 2003). In summary, it is currently thought that SIHI, LIHI and IHF are mediated through glutamatergic excitatory transcallosal M1-M1 connections, and that interhemispheric inhibition occurs through activation of interposed inhibitory interneurons in the M1 of the test hemisphere (Daskalakis et al., 2002; Ni et al., 2009).

Conditioning stimulation of various other cortical areas such as dorsal premotor cortex (PMd), ventral premotor cortex (PMv), supplementary motor area (SMA; Oliveri et al., 2003), and posterior parietal cortex (PPC) can also affect MEP amplitudes elicited by TS of the test M1. Anatomical connectivity of these areas with M1 is reported in animal studies (Muakkassa et al., 1979; Ghosh et al., 1987; Ghosh and Porter, 1988; Luppino et al., 1993; Stepniewska et al., 1993; Tokuno et al., 2000; Dum and Strick, 2005; Dea et al., 2016; Quessy et al., 2016), providing the rationale for testing effective connectivity in humans using pcTMS protocols. Since the ipsilateral PMd is adjacent to M1, revealing ipsilateral PMd-M1 connectivity was not straightforward due to difficulties in placing two coils appropriately on the scalp (Civardi et al., 2001; Koch et al., 2007b; Bäumer et al., 2009). More recently, Groppa et al. (2012) introduced a specially designed small coil with decentralized coil windings to overcome this problem, reporting MEP facilitation if CS was given $2.4-2.8 \mathrm{~ms}$ or $4.4 \mathrm{~ms}$ after the TS. In contrast, there is cumulative evidence on inhibitory effective connectivity between the contralateral PMd and M1. Mochizuki et al. reported MEP inhibition if the CS was given $8 \mathrm{~ms}$ prior to the TS (Mochizuki et al., 2004), which was confirmed by subsequent studies (Koch et al., 2007b, Ni et al., 2009

The SMA is another important secondary motor area, with predominantly facilitatory projections to M1 (Tokuno et al., 2000). Congruent with this observation, two pcTMS studies reported MEP facilitation by SMA conditioning. Arai et al. (2012) used a highly focal small CS coil over SMA to avoid current spread to the M1, and reported that relatively strong CS (140\% of active motor threshold) resulted in MEP facilitation at an ISI of 6 ms. This facilitation was coil-orientation specific and observed only if the induced current in the brain was directed from medial-to-lateral towards the stimulated SMA. Using a weaker CS, another study showed no effect on MEP amplitude but a facilitatory effect on SICF (Shirota et al., 2012) providing evidence that conditioning stimulation of SMA interacts with the excitatory interneuron circuitry in M1 responsible for the generation of I-waves.

PPC is a part of the fronto-parietal network that is important for visuomotor planning. Koch et al. revealed MEP facilitation at an ISI of $4 \mathrm{~ms}$, specifically at a CS intensity of $90 \%$ resting motor threshold, but not at lower or higher CS intensities (Koch et al., 2007a). A more recent study revealed both facilitatory and inhibitory effective connectivity, dependent on the exact PPC stimulation site (Karabanov et al., 2013), suggesting that different sub-divisions of the PPC play diverse roles in the fronto-parietal network.

For conditioning stimulation of the cerebellum, the main target is the cerebello-dentato-thalamo-motor cortical pathway. Within this network, Purkinje cells in the cerebellar cortex send inhibitory input to the dentate nucleus, which in turn has a di-synaptic excitatory connection with the contralateral M1 through the ventrolateral nucleus of the thalamus with a net inhibitory effect (Ito et al., 1970; Allen and Tsukahara, 1974). Ugawa et al. (1991, 1995) were the first to report cerebellar inhibition (CBI), probably activating this pathway. $\mathrm{CBI}$ is observed when the CS to the cerebellum precedes the TS of contralateral M1 by 5-7 ms, with a typical CS intensity of $90-95 \%$ of the active motor threshold to directly activate the pyramidal tract at the level of the pyramidal decussation using the double-cone coil. This hypothesis of net effect was approved by several cerebellar stimulation studies in patients with ataxia (Ugawa et al., 1994, 1997). A focal figure-of-eight coil would predominantly activate peripheral components of the cervicobrachial plexus near the coil rather than the cerebellum, resulting in another type of inhibition starting at slightly longer ISIs (7$8 \mathrm{~ms}$ ) than the $\mathrm{CBI}$, and potentially contaminating CBI at these longer ISIs (Werhahn et al., 1996). With enough caution about these factors, $\mathrm{CBI}$ can provide a unique opportunity to test effective connectivity from cerebellum to contralateral M1 through the cerebello-dentato-thalamo-motor cortical projection.

The recently developed multilocus TMS (mTMS; Koponen et al., 2018), which allows one to electronically adjust the location of the stimulated cortical location, will enable new kinds of pcTMS thanks to the possibility to stimulate also close-by cortical targets at programmable time intervals and intensities. Preliminary results show that (Nieminen et al., 2017) short-distance $(0-30 \mathrm{~mm}$ ) or lateral inhibition depends both on ISI and the distance between CS and TS targets. 


\subsection{Combined TMS approaches for testing cortico-cortical effective connectivity}

Effective connectivity includes a definition of causality, which cannot be provided by techniques like high-density EEG and fMRI per se. Obtaining measures of effective connectivity with highdensity EEG requires in fact complex causal models based on pre-existing data and the inferential power of such techniques on cortical effective connectivity relies on a priori assumptions about the involved network and the validity of the implemented model. In this perspective on the other hand, brain responses to TMS are intrinsically causal (Paus, 2005) even if not entirely "natural" and behavioral effects that follow the perturbation may be immediately detected/reported. Then, the online combination of EEG with TMS, due to the ability of EEG to detect changes of neuronal activities evoked by magnetic perturbation in a timescale of milliseconds, can return unprecedented hints on the functional properties of human cortical circuits in health and disease (Siebner et al., 2009; Ziemann, 2011).

Only when a TMS-compatible EEG amplifier is employed, electromagnetic artifacts caused by the TMS discharge are prevented (Virtanen et al., 1999; Ilmoniemi and Kičić, 2010). Besides the electromagnetic TMS-evoked artifacts, TEPs can reliably reflect genuine responses of cortical circuits to TMS provided that biological artifacts, such as somatosensory or auditory evoked potentials, are appropriately reduced or abolished (Gosseries et al., 2015) and state-of-the-art methodologies are applied to minimize confounding factors (Casarotto et al., 2010; Rogasch et al., 2014). Moreover, the TMS-evoked muscle artifacts constitute a major challenge with TMS-EEG when areas below cranial muscles are stimulated (Mutanen et al., 2013) as well as secondary to reflex muscle responses due to cranial nerves excitation. A large number of studies have been published as to how these artifacts could be filtered from the signal. One attractive alternative is Independent Component Analysis (ICA; Korhonen et al., 2011), but it suffers in this context from the fact that TEPs and muscle artifacts are not independent. Fortunately, more effective methods have been developed to solve the problem (e.g., Mäki and Ilmoniemi, 2011; Ilmoniemi et al., 2015; Mutanen et al., 2018). Finally, in order to obtain reproducible and reliable TMS-EEG measurements, it is necessary to use a neuronavigation system that allows to precisely target desired cortical locations, to keep the stimulation parameters constant over different sessions (Casarotto et al., 2010; Hannula and Ilmoniemi, 2017). TEPs can be used to reliably keep track of cortical excitability and effective connectivity in both research and clinical settings (Ziemann, 2011; Rossini et al., 2015).

Once the appropriate equipment is employed and the correct experimental procedures are implemented TMS-EEG coregistration (Cracco et al., 1989; Ilmoniemi et al., 1997) contributes estimating fundamental indices of cortical functioning (Bonato et al., 2006; Ilmoniemi and Kicic, 2010; Rogasch and Fitzgerald, 2013) and discriminating causal interactions from mere temporal correlations.

TMS-evoked EEG potentials (TEPs) occurring in the first 20$40 \mathrm{~ms}$ after the TMS pulse most likely reflect the responses of cortical circuits excited underneath the stimulator (Mueller et al., 2014; Li et al., 2017). On the contrary, later TEPs result from the propagation of the initial response to TMS to remote cortical circuits (Massimini et al., 2005; Bonato et al., 2006; Ferreri at al., 2011). Thus, to keep track of cortical excitability, defined as the electrical reactivity of the cerebral cortex to a direct perturbation, one should measure the slope and amplitude of the very early TEPs. To capture more global features of cortical excitability, the analysis of TEPs could be carried out also in the frequency and timefrequency domains. More in detail, TMS-EEG studies showed that the human cerebral cortical areas react differently during non-REM slow wave sleep compared to wakefulness (Massimini et al., 2005; Bergmann et al., 2012), that cortico-thalamic modules generate electrical responses with a regionally specific natural frequency (Rosanova et al., 2009; Ferrarelli et al., 2012), that cortical excitability changes are 'naturally' present during the day (Huber et al., 2013; Ly et al., 2016), during cortical development (Määttä et al., 2017) and physiological aging (Ferreri et al., 2017b). Moreover, TEPs are affected by CNS-active drugs (Premoli et al., 2014; Darmani et al., 2016; Premoli et al., 2017; Casarotto et al., 2019), alcohol use (Kähkönen et al., 2001) and abuse (Kaarre et al., 2018), and by psychiatric and neurological disorders, such as schizophrenia (Ferrarelli et al., 2012), depression (Canali et al., 2015), Alzheimer's disease (Casarotto et al., 2011; Ferreri et al., 2016), or epilepsy (Kimiskidis et al., 2017).

Since the beginning, TEPs were used to measure cortico-cortical connectivity, such as interhemispheric propagation time (Cracco et al., 1989). However, measuring effective connectivity within cortical circuits by means of TMS-EEG is challenging since the local and remote cortical responses to TMS arise from excitation of axons in both orthodromic and antidromic directions regardless of their physiological "directionality" (Ilmoniemi and Kicic, 2010). Most important, localization of scalp EEG potentials is strongly affected by electrode positioning for TEP recordings and volume conduction and may lead to computation of wrong connectivity patterns (van den Broek et al., 1998; Cohen, 2017). For this reason, a reliable assessment of cortical effective connectivity must be conducted at the level of cortical sources that generate TEPs (Schoffelen and Gross, 2009). For instance, in the first seminal TMS-EEG study (Ilmoniemi et al., 1997), TEPs after stimulation of the primary motor and visual cortical areas were analyzed at the scalp and cortical levels providing the first non-invasive measures of cortical excitability and effective connectivity in humans. Later on, using a similar approach, Massimini and colleagues observed that cortical effective connectivity collapses in healthy subjects during non-REM slow wave sleep, when consciousness physiologically fades away, despite preserved cortical excitability (Massimini et al., 2005). The same research group has developed a semi-automatic procedure to analyze TMS-EEG data, which provides three indices as readouts: significant current density (SCD), phase-locking (PL), and significant current scattering (SCS) (Casali et al., 2010). They showed that cortical effective connectivity breaks down during unconscious states induced by deep sedation (Ferrarelli et al., 2010; Sarasso et al., 2015) or by severe brain lesions (Rosanova et al., 2012; Ragazzoni et al., 2013; Rosanova et al., 2018). Accordingly, indices of cortical effective connectivity based on TMS-EEG measurements recover when consciousness returns, but behavioral responses are still absent or inconsistent, such as during dreaming (Massimini et al., 2010), ketamine anesthesia (Sarasso et al., 2015), Minimally Conscious State (MCS), and emergence from MCS (Fig. 12; adapted from Rosanova et al., 2012).

Another metric derived from TMS-EEG measurements is able to detect the joint presence of cortical differentiation and cortical integration (Casali et al., 2013). The index, termed Perturbational Complexity Index (PCI) is computed in two steps: (i) a perturbation of the cortex with TMS to trigger causal and distributed interactions within brain circuits (ii) a compression of the cortical responses computed at the source level to measure their algorithmic complexity (Casarotto et al., 2016).

Assessment of cortical effective connectivity based on TMS-EEG recordings can also be applied to address cognitive neuroscience issues such as the mechanisms underlying attention shifts (Morishima et al., 2009), or the modulation of language circuits by other non-invasive brain stimulation techniques (Pisoni et al., 2018). On the same line, future studies should fully exploit the potential of TMS-EEG to extensively and automatically map corti- 

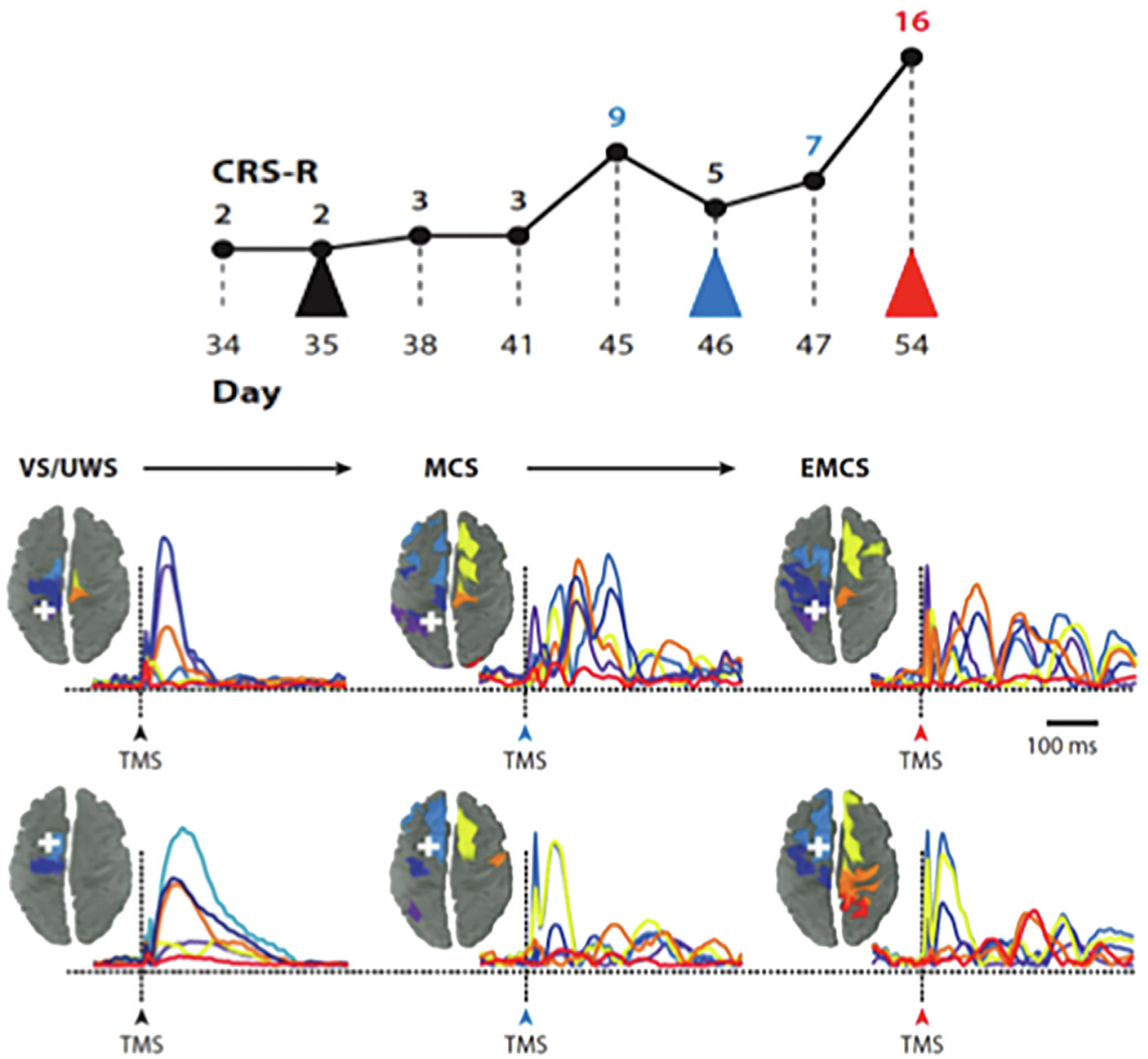

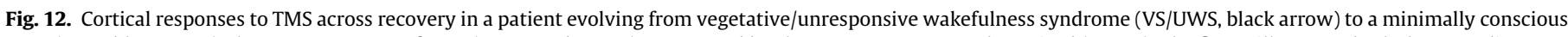

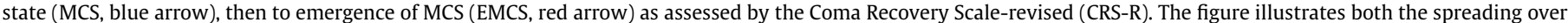

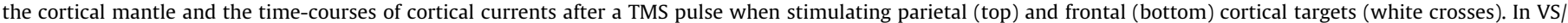
UWS, the response is local and simple, while involves different cortical sources at different times in MCS and EMCS. Figure adapted from Rosanova et al. (2012).

cal circuits (Harquel et al., 2016) in order to measure effective connectivity in dysfunctional brains (Ferrarelli et al., 2008, Adhikari et al., 2017). This approach can hopefully pave the way to novel rehabilitative treatments for brain disorders that will be aimed at promoting adaptive rather than maladaptive processes in response to neurological or psychiatric diseases (Fornito et al., 2015).

A major challenge with TMS-EEG is constituted by the huge muscle artifacts when areas below cranial muscles are stimulated (Mutanen et al., 2013) as well as secondary to reflex muscle responses due to cranial nerves excitation. A large number of studies have been published as to how these artifacts could be filtered from the signal. One attractive alternative is Independent Component Analysis (ICA; Korhonen et al., 2011), but it suffers in this context from the fact that TEPs and muscle artifacts are not independent. Fortunately, more effective methods have been developed to solve the problem (e.g., Mäki and Ilmoniemi, 2011; Ilmoniemi et al., 2015; Mutanen et al., 2018).

Another TMS approach is its combination with fMRI for the exploration of connectivity. As compared to EEG, relevant advantages of TMS-fMRI include better spatial resolution, and the option to explore also cortico-subcortical connectivity with enhanced reliability. The main shortcoming of TMS-fMRI is however its relatively poor temporal resolution, which limits opportunities to explore fast oscillation-based dynamic alterations of connectivity with this technique. This does however not exclude to obtain information about dynamic task-related alterations of connectivity in general, as shown for motor cortical networks (Bestmann et al.,
2008), but also the interplay between large-scale network interactions relevant for cognitive and emotional processing (Chen et al., 2013). Thus both tools fulfill partially complimentary needs, regarding exploration of functional connectivity in the human brain, and combination of EEG with fMRI and brain stimulation might be a future direction worthwhile to explore.

Repetitive (rTMS) and patterned type TMS (i.e. theta-burst TMS) is now considered an advantageous probe to test brain networks underlying cognitive functions since the use of TMS follows the rule of inference. If cortical area $A$ is involved in cognitive process $B$ and is not involved in process $C$, perturbation of the activity of area $A$ will result in altered performance in $B$ and not $C$. Thus, for deductive reasoning, area $A$ plays a causal role in the performance of B. Moreover, TMS can be safely repeated in subjects on different occasions, eventually allowing an intra-lab or between-lab retest of a given experimental hypothesis. Finally, in some specific fields, as memory tasks requiring a two-stage cognitive process (i.e., encoding and later retrieval of items), TMS allows to tease apart the effects on one of these two tasks more easily than in the case of lesion studies (e.g., Rossi et al., 2001, 2004).

Because information processing of higher brain functions is integrated within several parallel distributed networks involving "nodes" in many cortical areas, a single pulse is often inadequate to interfere with the brain activity at a behaviorally relevant level, although a very first example of TMS use outside the motor cortex used single pulses to transiently suppress the visual perception, by stimulating the occipital cortex about $80-100 \mathrm{msec}$ after the pre- 
sentation of the visual stimulus (Amassian et al., 1989) or producing a transient "neglect" to a sensory hand stimulation (Oliveri et al., 1999, 2000). In this context TMS may be used as a tool to investigate and understand the role and timing of the involvement of a target area in a specific performance (Walsh and Cowey, 2000), the contribution of different sites to different aspects of a cognitive function (Terao et al., 2001; Robertson et al., 2003), the relative timing of the contribution of two or more areas to task performance and the function of intracortical and transcallosal connectivity (Jahanshahi and Rothwell, 2000). In short, what information is processed in a given brain network, and when does this processing occur. In general, the possibility of understanding the location, timing (i.e., cognitive chronometry) (Walsh et al., 2006) and functional relevance of a given node activity within a network underlying cortical functions makes rTMS an essential technique mainly in perception and cognitive research.

In general, it should be taken into account that at present the application of non-invasive brain stimulation approaches - including TMS, but also tDCS/tACS, which is introduced in the next section - to explore functional connectivity is still in its early days. Major approaches include exploration of spatio-temporal maps of brain activity alterations elicited by stimulation of a specific area, and refer to the whole brain, or specific interactions between a seed region of interest, and remaining structures. Network stimulation involving not only stimulation of a single hub, which indirectly alters network activity, but of a larger set of relevant areas, has rarely been conducted. Double coil approaches for TMS, and specific tACS protocols (see below) are exceptions, and might pave the way to more complex brain stimulation protocols in future to explore the dynamics and causality of functional connectivity in larger detail.

\section{5. $t D C S$ and $t A C S$}

Tonic or oscillatory stimulation with weak electrical currents, labelled transcranial direct and alternating current stimulation (tDCS, tACS), which are subsumed under the general term transcranial electrical stimulation (tES), induces alterations of cortical activity and excitability via subthreshold modulation of neuronal membrane potentials; prolonged stimulation can generate neuroplastic after-effects (Nitsche et al., 2008; Stagg and Nitsche, 2011). However, tES can be also valuable in inducing and probing connectivity of the human brain, including its relevance for psychological and behavioral processes. Hereby, (a) controlled modulation of regional cortical activity and excitability by tES in combination with neuroimaging or TMS can reveal specific functional connections of the targeted areas, (b) combination of stimulation with cognitive or motor processes and neuroimaging approaches can reveal the relevance of the targeted area for taskrelated functional connections, and (c) tES based on connectivity data obtained during task performance can be used to explore the causal relevance of respective connections for performance.

For revealing functional connections of a targeted cortical area tDCS studies have been conducted, mainly to explore motor network connectivity. Combination of tDCS with fMRI showed that activation of the primary motor cortex via tDCS enhanced functional connectivity of this target area with the premotor, and parietal cortex, but also with subcortical areas like the ipsilateral thalamus (Polania et al., 2011b, 2012b). Furthermore, combining tDCS with TMS demonstrated that tDCS over the premotor cortex can alter SICF and SICI presumably by altering premotor-M1 connectivity, while corticospinal excitability as tested by singlepulse TMS over M1, which most likely is elicited by stimulation of intrinsic pyramidal tract neurons, was not altered (Boros et al., 2008). Similar effects on M1 excitability, tested by alterations of parieto-motor effective connectivity by a double coil approach, were revealed for posterior parietal cortex tDCS (Rivera-Urbina et al., 2015). One advantage to use brain stimulation approaches such as tDCS to probe connectivity is their specificity, i.e., the restrictedness of stimulation to a pre-defined target area, as compared to task-related activation, which in most cases goes along with multi-modal brain area activation. This requires, however, specific intervention protocols, including computational modelbased stimulation approaches, and use of small area electrodes (which, however, decreases the depth and amount of current reaching the underlying cortex). Unfortunately, most of the studies did not use a control condition exploring eventual effects of real stimulation of a non-target brain area in order to demonstrate a site-specific effect. Taking these caveats into account, relative specific effects of the stimulation, dependent on electrode position, can be expected, and help to define connectivity of the targeted brain network (Nitsche et al., 2007; Boros et al., 2008; RiveraUrbina et al., 2015). Beyond these purely physiological measures, tDCS can also be used to explore the relevance of a targeted area for task-related connectivity. The concept here is to modulate activity and/or excitability of the target area, and then to explore effects on task-related functional connectivity by functional imaging approaches. Polania et al. (2011a) conducted an experiment, in which resting state EEG and task-related EEG during finger tapping were obtained before and after anodal tDCS over the primary motor cortex. They showed that tDCS alone had only minor effects on connectivity of the resting state EEG, but that task-related connectivity, specifically in the high gamma frequency band, between motor, premotor, and sensorimotor areas were enhanced after tDCS. For all other frequency bands, the tDCS effects on connectivity were less clear and not focused on areas involved in motor task performance. Thus, tDCS can be used as a probe to specifically enhance task-relevant functional connectivity.

Finally, tES can be used as a tool to specifically modulate physiological processes underlying functional connectivity, and thus probe the relevance of connectivity for psychological and behavioral processes. For functional connectivity, temporal association of oscillatory brain activity, so-called "binding" processes, are thought to play a crucial role. Thus, synchronizing the cycling firing of remote, but functionally connected areas should improve performance, whereas desynchronization of this distributed activity should have detrimental effects. For such a modulation of oscillatory brain activity, transcranial alternating current stimulation (tACS) is a versatile tool. For delayed letter discrimination, i.e., a working memory process, it has been suggested that lefthemispheric fronto-parietal interactions play a crucial role. Polania et al. (2012a, 2012b) tested the causal relevance of this connection for working memory performance using tACS. During task performance, activity of fronto-parietal areas in the theta frequency band was enhanced. Furthermore, about $200 \mathrm{~ms}$ after stimulus presentation, activity in this frequency range started to synchronize between fronto-parietal areas. Moreover, stronger synchronization was associated with better task performance. Thus, it was hypothesized that not only enhanced theta activity of left hemispheric prefrontal and parietal areas, but also their synchronization was causally related to task performance. This was directly tested by delivering tACS over the prefrontal and parietal target areas during task performance. Real or sham tACS with a frequency of $6 \mathrm{~Hz}$ was applied in phase or out of phase, and thus in synchronized or desynchronized mode. In accordance with expectations, synchronized tACS enhanced, whereas desynchronized tACS reduced working memory performance relative to the sham stimulation condition. Moreover, these effects were specific for theta frequency stimulation, since a control experiment, in which gamma frequency tACS was applied, had no effect. This paradigmatic experiment demonstrated how tACS can be used to explore the relevance of functional (in this case frequency-specific) con- 
nectivity in a distributed neuronal network for task performance. Ideally, the stimulation experiment would have included also EEG recordings concurrent with tACS (Helfrich et al., 2014; Voss et al., 2014); the combination of tACS with EEG recordings will make it possible to explore physiological and cognitive stimulation effects in the same experiment, and will help to derive causality between connectivity and function more directly in future studies.

Taken together, the examples given here posit tES as a valuable tool for exploring connectivity in the human brain, including its behavioral relevance. tES can be used to induce/enhance connectivity originating from a specific area and, thus, to characterize connections independent from complex, often multi-modal, taskrelated activation. It furthermore can be useful to explore the contribution of a target area to a task-dependently activated network, and it can be applied to explore the causal relevance of functional connectivity for task performance, including specific features, such as frequency-dependency, and mode of synchronization. One important aspect of tES is its primary neuromodulatory effect. In contrast to TMS, it does not induce, but modifies spontaneous cerebral activity. Dependent on the context to be explored, this can be advantageous or disadvantageous. Thus, the non-disruptive online effects of tES might be crucial for its bi-directional effects on task performance, but, together with limitations regarding temporal and spatial resolution, might limit its suitability regarding physiological determination of connectivity. Future protocols, including closed-loop, and multi-electrode systems, might further enhance precision of these tools, and enable exploration of larger and more complex functionally connected networks in vivo in the human brain.

\section{Conclusions}

This review provides an extensive, multimodal and updated approach to the topic of methods for the exploration of brain connectivity, with a consideration of the strengths and weaknesses of each technology. A complex and multifaceted variety of aspects including those concerning structural, functional, effective, timevarying and dynamic brain connections cannot be approached and solved with a single method, but needs multiple and integrated methodologies for solving its individual facets. Within this line a multidisciplinary team of researchers are needed in order to select the optimal methods to track the scientific targets in the most appropriate way. Knowledge of the structural connections logically comes first; what parts of the brain are anatomically connected. As noted here, information about structure can build from microscopic to macroscopic scale. On that basis, it is possible to explore and understand the functional connectivity. Moreover, information flow will vary dynamically with brain state. Much information can be, and is being collected, in the resting state, but ultimately it will be crucial to know information flow related to different tasks and behaviors. As has been pointed out, since the brain operates in networks, it will be necessary to have descriptors of network operation to fully understand the neural processing. With this type of approach, future studies and an enriching "connection" within the research community will enable neuroscientists to disentangle the inner mechanisms regulating connectivity architecture of the major brain activities, including learning, memory, mood level, emotional expression, language, task-related skills and other domains, both in health and in neuropsychiatric diseases.

Looking at the functioning brain as a "society" of dynamically interconnected neuronal assemblies represents a change of paradigm not only in neuroscientific research, but also (or even mainly) in clinical neurosciences. In the near future it will be possible to disentangle "good" (i.e. by recognizing online the presence of opti- mal network architecture for learning, memory, task-related function) and "aberrant" networks (i.e. those sustaining a symptom like an epileptic spike, a dystonic movement, a behavioral/cognitive dysfunction) and to tailor therapeutic and rehabilitative approaches having a central "marker" to measure their efficacy and to better personalize the interventions of cure.

\section{Disclosures}

None of the authors have potential conflicts of interest to be disclosed.

\section{References}

Adhikari MH, Hacker CD, Siegel JS, Griffa A, Hagmann P, Deco G, et al. Decreased integration and information capacity in stroke measured by whole brain models of resting state activity. Brain 2017;140:1068-85.

Ahlfors SP, Simpson GV, Dale AM, Belliveau JW, Liu AK, Korvenoja A, et al Spatiotemporal activity of a cortical network for processing visual motion revealed by MEG and fMRI. J Neurophysiol 1999;82:2545-55.

Allen GI, Tsukahara N. Cerebrocerebellar communication systems. Physiol Rev 1974;54:957-1006.

Amassian VE, Cracco RQ, Maccabee PJ, Cracco JB, Rudell A, Eberle L. Suppression of visual perception by magnetic coil stimulation of human occipital cortex. Electroencephalogr Clin Neurophysiol 1989;74:458-62.

Amassian VE, Stewart M, Quirk GJ, Rosenthal JL. Physiological basis of motor effects of a transient stimulus to cerebral cortex. Neurosurgery 1987;20:74-93.

Aoki Y, Ishii R, Pascual-Marqui RD, Canuet L, Ikeda S, Hata M, et al. Detection of EEGresting state independent networks by eLORETA-ICA method. Front Hum Neurosci 2015;9:31.

Arai N, Lu M-K, Ugawa Y, Ziemann U. Effective connectivity between human supplementary motor area and primary motor cortex: a paired-coil TMS study. Exp Brain Res 2012;220:79-87.

Arfanakis K, Cordes D, Haughton VM, Moritz CH, Quigley MA, Meyerand ME. Combining independent component analysis and correlation analysis to probe interregional connectivity in fMRI task activation datasets. Magn Reson Imaging 2000; 18:921-30.

Aydore S, Pantazis D, Leahy RM. A note on the phase locking value and its properties. Neuroimage 2013;74. 231-44.C.

Avena-Koenigsberger A, Misic B, Sporns O. Communication dynamics in complex brain networks. Nat Rev Neurosci 2017;19:17-33.

Babiloni C, Barry R, Başar E, Blinowska KJ, Drinkenburg WHIM, Knight R, et al. IFCN guidelines for topographic and frequency analysis of resting state electroencephalographic rhythms. Clin Neurophysiol 2018;129(Suppl 1): e208.

Bandettini PA. Neuronal or hemodynamic? Grappling with the functional MRI signal. Brain Connect 2014;4:487-98.

Barker AT, Jalinous R, Freeston IL. Non-invasive magnetic stimulation of human motor cortex. Lancet 1985;1:1106-7.

Barry RJ, De Blasio FM, Borchard JP. Sequential processing in the equiprobable auditory Go/NoGo task: children vs. adults. Clin Neurophysiol 2014;125:1995-2006.

Basser PJ, Mattiello J, LeBihan D. MR diffusion tensor spectroscopy and imaging. Biophys J 1994;66:259-67.

Bassett DS, Bullmore E, Verchinski BA, Mattay VS, Weinberger DR, MeyerLindenberg A. Hierarchical organization of human cortical networks in health and schizophrenia. J Neurosci 2008;28:9239-48.

Bassett DS, Bullmore E. Small-world brain networks. Neuroscientist 2006;12:512-23.

Bäumer T, Schippling S, Kroeger J, Zittel S, Koch G, Thomalla G, et al. Inhibitory and facilitatory connectivity from ventral premotor to primary motor cortex in healthy humans at rest - a bifocal TMS study. Clin Neurophysiol 2009; 120:1724-31.

Behrens TE, Sporns O. Human connectomics. Curr Opin Neurobiol 2012;22:144-53.

Bell AJ, Sejnowski TJ. An information-maximization approach to blind separation and blind deconvolution. Neural Comput 1995;7:1129-59.

Bentivoglio M, Kuypers HGJM, Catsman-Berrevoets CE, Loewe H, Dann O. Two new fluorescent retrograde neuronal tracers which are transported over long distances. Neurosci Lett 1980;18:25-30.

Bentivoglio M, Mazzarello P. Chapter 12: the anatomical foundations of clinical neurology. Handb Clin Neurol 2010;95:149-68.

Bentivoglio M. The discovery of axonal transport. Brain Res Bull 1999;50:383-4.

Bergmann TO, Mölle M, Schmidt MA, Lindner C, Marshall L, Born J, et al. EEG-guided transcranial magnetic stimulation reveals rapid shifts in motor cortical excitability during the human sleep slow oscillation. J Neurosci 2012;32:243-53.

Bestmann S, Swayne O, Blankenburg F, Ruff CC, Haggard P, Weiskopf N, Josephs O, Driver J, Rothwell JC, Ward NS. Dorsal premotor cortex exerts state-dependent causal influences on activity in contralateral primary motor and dorsal premotor cortex. Cereb Cortex 2008;18:1281-91. 
Biswal B, Yetkin FZ, Haughton VM, Hyde JS. Functional connectivity in the motor cortex of resting human brain using echo-planar MRI. Magn Reson Med 1995;34:537-41.

Bonato C, Miniussi C, Rossini PM. Transcranial magnetic stimulation and cortical evoked potentials: a TMS/EEG co-registration study. Clin Neurophysiol 2006;117:1699-707.

Bönstrup M, Feldheim J, Heise K, Gerloff C, Hummel FC. The control of complex finger movements by directional information flow between mesial frontocentral areas and the primary motor cortex. Eur J Neurosci 2014;40:2888-97.

Bönstrup M, Schulz R, Feldheim J, Hummel FC, Gerloff C. Dynamic causal modelling of EEG and fMRI to characterize network architectures in a simple motor task. Neuroimage 2016;124:498-508.

Bönstrup M, Schulz R, Schön G, Cheng B, Feldheim J, Thomalla G, et al. Parietofrontal network upregulation after motor stroke. Neuroimage Clin 2018;18:720-9.

Boros K, Poreisz C, Munchau A, Paulus W, Nitsche MA. Premotor transcranial direct current stimulation (tDCS) affects primary motor excitability in humans. Eur J Neurosci 2008;27:1292-300.

Boyden ES, Zhang F, Bamberg E, Nagel G, Deisseroth K. Millisecond-timescale, genetically targeted optical control of neural activity. Nat Neurosci 2005;8:1263-8.

Bressler SL, Coppola R, Nakamura R. Episodic multiregional cortical coherence at multiple frequencies during visual task performance. Nature 1993;366: 153-6.

Bressler SL, Menon V. Large-scale brain networks in cognition: emerging methods and principles. Trends Cogn Sci 2010;14:277-90.

Buchel C, Friston KJ. Modulation of connectivity in visual pathways by attention: cortical interactions evaluated with structural equation modelling and fMRI. Cereb Cortex 1997;7:768-78.

Bullmore E, Sporns O. Complex brain networks: graph theoretical analysis of structural and functional systems. Nat Rev Neurosci 2009;10:186-98.

Buzsaki G, Draguhn A. Neuronal oscillations in cortical networks. Science 2004;304:1926-9.

Caiafa CF, Cichocki A. Computing sparse representations of multidimensional signals using kronecker bases. Neural Comput 2013;25:186-220.

Caiafa CF, Pestilli F. Multidimensional encoding of brain connectomes. Sci Rep 2017:7:11491.

Cajal SR. Cajal's histology of the nervous system of man and vertebrates. New York: Oxford University Press; 1995.

Cajal SR. Histologie du système nerveux de l'homme et des vertébrés. Paris: Maloine; 1909.

Canali P, Sarasso S, Rosanova M, Casarotto S, Sferrazza-Papa G, Gosseries O, et al. Shared reduction of oscillatory natural frequencies in bipolar disorder, major depressive disorder and schizophrenia. J Affect Disord 2015;184:111-5.

Canuet L, Ishii R, Pascual-Marqui RD, Iwase M, Kurimoto R, Aoki Y, et al. Restingstate EEG source localization and functional connectivity in schizophrenia-like psychosis of epilepsy. PLoS ONE 2011;6:e27863.

Casali AG, Casarotto S, Rosanova M, Mariotti M, Massimini M. General indices to characterize the electrical response of the cerebral cortex to TMS. Neuroimage 2010;49:1459-68.

Casali AG, Gosseries O, Rosanova M, Boly M, Sarasso S, Casali KR, et al. A theoretically based index of consciousness independent of sensory processing and behavior. Sci Transl Med 2013;5:198ra05.

Casarotto S, Comanducci A, Rosanova M, Sarasso S, Fecchio M, Napolitani M, et al. Stratification of unresponsive patients by an independently validated index of brain complexity. Ann Neurol 2016;80:718-29.

Casarotto S, Määttä S, Herukka SK, Pigorini A, Napolitani M, Gosseries O, et al. Transcranial magnetic stimulation-evoked EEG/cortical potentials in physiological and pathological aging. NeuroReport 2011;22:592-7.

Casarotto S, Romero Lauro LJ, Bellina V, Casali AG, Rosanova M, Pigorini A, et al. EEG responses to TMS are sensitive to changes in the perturbation parameters and repeatable over time. PLoS ONE 2010;5:e10281.

Casarotto S, Turco F, Comanducci A, Perretti A, Marotta G, Pezzoli G, et al. Excitability of the supplementary motor area in Parkinson's disease depends on subcortical damage. Brain Stimul 2019;12:152-60.

Chen AC, Oathes DJ, Chang C, Bradley T, Zhou ZW, Williams LM, et al. Causal interactions between fronto-parietal central executive and default-mode networks in humans. Proc Natl Acad Sci USA 2013;110:19944-9.

Cash RF, Ziemann U, Murray K, Thickbroom GW. Late cortical disinhibition in human motor cortex: a triple-pulse transcranial magnetic stimulation study. J Neurophysiol 2010;103:511-8.

Cash RF, Ziemann U, Thickbroom GW. Inhibitory and disinhibitory effects on I-wave facilitation in motor cortex. J Neurophysiol 2011;105:100-6.

Chen CC, Kiebel SJ, Friston KJ. Dynamic causal modelling of induced responses, Neuroimage 2008a:41:1293-312.

Chen R, Cros D, Curra A, Di Lazzaro V, Lefaucheur JP, Magistris MR, et al. The clinical diagnostic utility of transcranial magnetic stimulation: report of an IFCN committee. Clin Neurophysiol 2008b;119:504-32.

Chen R, Yung D, Li J-Y. Organization of ipsilateral excitatory and inhibitory pathways in the human motor cortex. J Neurophysiol 2003;89:1256-64.

Chung K, Wallace J, Kim S-Y, Kalyanasundaram S, Andalman AS, Davidson TJ, et al. Structural and molecular interrogation of intact biological systems. Nature 2013;497:332-7.

Cichocki A, Mandic D, Phan AH, Caiafa C, Zhou G, Zhao Q et al. Tensor decompositions for signal processing applications: from two-way to multiway component analysis. IEEE Signal Process Mag 2015;232:145-63.
Civardi C, Cantello R, Asselman P, Rothwell JC. Transcranial magnetic stimulation can be used to test connections to primary motor areas from frontal and medial cortex in humans. Neuroimage 2001;14:1444-53.

Classen J, Gerloff C, Honda M, Hallett M. Integrative visuomotor behavior is associated with interregionally coherent oscillations in the human brain. J Neurophysiol 1998;79:1567-73.

Cohen MX. Where does EEG come from and what does it mean? Trends Neurosci 2017;40:208-18.

Connors BW, Malenka RC, Silva LR. Two inhibitory postsynaptic potentials, and GABAA and GABAB receptor- mediated responses in neocortex of rat and cat. J Physiol 1988;406:443-68.

Colclough GL, Woolrich MW, Tewarie PK, Brookes MJ, Quinn AJ, Smith SM. How reliable are MEG resting-state connectivity metrics? Neuroimage 2016;138:284-93.

Cordes D, Haughton V, Carew JD, Arfanakis K, Maravilla K. Hierarchical clustering to measure connectivity in fMRI resting-state data. Magn Reson Imaging 2002;20:305-17.

Cowan WM, Gottlieb DI, Hendrickson AE, Price JL, Woolsey TA. The autoradiographic demonstration of axonal connections in the central nervous system. Brain Res 1972;37:21-51.

Cracco RQ, Amassian VE, Maccabee PJ, Cracco JB. Comparison of human transcallosal responses evoked by magnetic coil and electrical stimulation. Electroencephalogr Clin Neurophysiol 1989;74:417-24.

Crossley NA, Fox PT, Bullmore ET. Meta-connectomics: human brain network and connectivity meta-analyses. Psychol Med 2016;46:897-907.

Curio G, Mackert BM, Burghoff M, Koetitz R, Abraham-Fuchs K, Härer W. Localization of evoked neuromagnetic $600 \mathrm{~Hz}$ activity in the cerebra somatosensory system. Electroencephalogr Clin Neurophysiol 1994;91:483-7.

Daducci A, Dal Palù A, Lemkaddem A, Thiran JP. COMMIT: convex optimization modeling for microstructure informed tractography. IEEE Trans Med Imaging 2015;34:246-57.

Darmani G, Zipser CM, Böhmer GM, Deschet K, Müller-Dahlhaus F, Belardinelli P, et al. Effects of the selective alpha5-GABAAR antagonist S44819 on excitability in the human brain: a TMS-EMG and TMS-EEG Phase I Study. J Neurosci 2016;36:12312-20.

Daskalakis ZJ, Christensen BK, Fitzgerald PB, Roshan L, Chen R. The mechanisms of interhemispheric inhibition in the human motor cortex. J Physiol 2002;543:317-26.

David O, Kiebel SJ, Harrison LM, Mattout J, Kilner JM, Friston KJ. Dynamic causa modeling of evoked responses in EEG and MEG. Neuroimage 2006;30:1255-72.

de Haan W, Pijnenburg YA, Strijers RL, van der Made Y, van der Flier WM, Scheltens $P$, et al. Functional neural network analysis in frontotemporal dementia and Alzheimer's disease using EEG and graph theory. BMC Neurosci 2009;10:101.

Dea M, Hamadjida A, Elgbeili G, Quessy S, Dancause N. Different patterns of cortica inputs to subregions of the primary motor cortex hand representation in Cebus apella. Cereb Cortex 2016;26:1747-61.

Deisseroth K, Feng G, Majewska AK, Miesenböck G, Ting A, Schnitzer MJ. Nextgeneration optical technologies for illuminating genetically targeted brain circuits. J Neurosci 2006;26:10380-6.

Deisseroth K. Optogenetics: 10 years of microbial opsins in neuroscience. Nat Neurosci 2015;18:1213-25.

Deisz RA. The GABA(B) receptor antagonist CGP 55845A reduces presynaptic GABA (B) actions in neocortical neurons of the rat in vitro. Neuroscience 1999;93:1241-9.

Delbeke J, Hoffman L, Mols K, Braeken D, Prodanov D. And then there was light: perspectives of optogenetics for deep brain stimulation and neuromodulation. Front Neurosci 2017;11:663.

Devanne H, Lavoie BA, Capaday C. Input-output properties and gain changes in the human corticospinal pathway. Exp Brain Res 1997;114:329-38.

Di Lazzaro V, Oliviero A, Mazzone P, Pilato F, Saturno E, Insola A, et al. Direct demonstration of long latency cortico-cortical inhibition in normal subjects and in a patient with vascular parkinsonism. Clin Neurophysiol 2002;113:1673-9.

Di Lazzaro V, Oliviero A, Profice P, Saturno E, Pilato F, Insola A, et al. Comparison of descending volleys evoked by transcranial magnetic and electric stimulation in conscious humans. Electroencephalogr Clin Neurophysiol 1998a:109:397-401.

Di Lazzaro V, Pilato F, Dileone M, Profice P, Ranieri F, Ricci V, et al. Segregating two inhibitory circuits in human motor cortex at the level of GABAA receptor subtypes: a TMS study. Clin Neurophysiol 2007;118:2207-14.

Di Lazzaro V, Restuccia D, Oliviero A, Profice P, Ferrara L, Insola A, et al. Magnetic transcranial stimulation at intensities below active motor threshold activates intracortical inhibitory circuits. Exp Brain Res 1998b;119:265-8.

Dimitriadis SI, Drakesmith M, Bells S, Parker GD, Linden DE, Jones DK. Improving the reliability of network metrics in structural brain networks by integrating different network weighting strategies into a single graph. Front Neurosc 2017; 11:694.

Dimitriadis SI, Routley B, Linden DE, Singh KD. Reliability of static and dynamic network metrics in the resting-state: A MEG-beamformed connectivity analysis. Front Neurosci 2018;12:506.

Dum RP, Strick PL. Frontal lobe inputs to the digit representations of the moto areas on the lateral surface of the hemisphere. J Neurosci 2005;25:1375-86.

Engel AK, Gerloff C, Hilgetag CC, Nolte G. Intrinsic coupling modes: multiscale interactions in ongoing brain activity. Neuron 2013;80:867-86.

Engel AK, Konig P, Kreiter AK, Singer W. Interhemispheric synchronization of oscillatory neuronal responses in cat visual cortex. Science 1991;252:1177-9.

Ferbert A, Priori A, Rothwell JC, Day BL, Colebatch JG, Marsden CD. Interhemispheric inhibition of the human motor cortex. J Physiol 1992;453:525-46. 
Ferrarelli F, Massimini M, Peterson MJ, Riedner BA, Lazar M, Murphy MJ, et al. Reduced evoked gamma oscillations in the frontal cortex in schizophrenia patients: a TMS/EEG study. Am J Psychiatry 2008;165:996-1005.

Ferrarelli F, Massimini M, Sarasso S, Casali A, Riedner BA, Angelini G, et al. Breakdown in cortical effective connectivity during midazolam-induced loss of consciousness. Proc Natl Acad Sci USA 2010;107:2681-6.

Ferrarelli F, Sarasso S, Guller Y, Riedner BA, Peterson MJ, Bellesi M, et al. Reduced natural oscillatory frequency of frontal thalamocortical circuits in schizophrenia. Arch Gen Psych 2012;69:766-74.

Ferreri F, Guerra A, Vollero L, Ponzo D, Maatta S, Mervaala E, et al. Age-related changes of cortical excitability and connectivity in healthy humans: noninvasive evaluation of sensorimotor network by means of TMS-EEG Neuroscience 2017a;357:255-63.

Ferreri F, Pasqualetti P, Määttä S, Ponzo D, Ferrarelli F, Tononi G, et al. Human brain connectivity during single and paired pulse transcranial magnetic stimulation. Neuroimage 2011:54:90-102.

Ferreri F, Vecchio F, Ponzo D, Pasqualetti P, Rossini PM. Time-varying coupling of EEG oscillations predicts excitability fluctuations in the primary motor cortex as reflected by motor evoked potentials amplitude: an EEG-TMS study. Hum Brain Mapp 2014;35:1969-80.

Ferreri F, Vecchio F, Guerra A, Miraglia F, Ponzo D, Vollero L, et al. Age related differences in functional synchronization of EEG activity as evaluated by means of TMS-EEG coregistrations. Neurosci Lett 2017b;647:141-6.

Ferreri F, Vecchio F, Vollero L, Guerra A, Petrichella S, Ponzo D, et al. Sensorimotor cortex excitability and connectivity in Alzheimer's disease: a TMS-EEG Coregistration study. Hum Brain Mapp 2016;37:2083-96.

Fields RD. A new mechanism of nervous system plasticity: activity-dependent myelination. Nat Rev Neurosci 2015;16:756-67.

Fields RD. White matter in learning, cognition and psychiatric disorders. Trends Neurosci 2008a;31:361-70.

Fields RD. White matter matters. Sci Am 2008b;298:54-61.

Finger H, Bonstrup M, Cheng B, Messe A, Hilgetag C, Thomalla G, et al. Modeling of large-scale functional brain networks based on structural connectivity from DTI: comparison with EEG derived phase coupling networks and evaluation of alternative methods along the modeling path. PLoS Comput Biol 2016;12: e1005025.

Fink RP, Heimer L. Two methods for selective silver impregnation of degenerating axons and their synaptic endings in the central nervous system. Brain Res 1967;4:369-74.

Finn ES, Shen X, Scheinost D, Rosenberg MD, Huang J, Chun MM, et al. Functiona connectome fingerprinting: identifying individuals using patterns of brain connectivity. Nat Neurosci 2015;18:1664-71.

Fornito A, Zalesky A, Breakspear M. The connectomics of brain disorders. Nat Rev Neurosci 2015;16:159-72.

Fransson P. Spontaneous low-frequency BOLD signal fluctuations: an fMRI investigation of the resting-state default mode of brain function hypothesis. Hum Brain Mapp 2005;26:15-29.

Fries P. Rhythms for cognition: communication through coherence. Neuron 2015;88:220-35.

Friston KJ, Harrison L, Penny W. Dynamic causal modelling. Neuroimage 2003;19:1273-302.

Friston KJ, Kahan J, Biswal B, Razi A. A DCM for resting state fMRI. Neuroimage 2014;94:396-407.

Friston KJ, Li B, Daunizeau J, Stephan KE. Network discovery with DCM. Neuroimage 2011;56:1202-21.

Fritzsch B, Muirhead KA, Feng F, Gray BD, Ohlsson-Wilhelm BM. Diffusion and imaging properties of three new lipophilic tracers, NeuroVue Maroon, NeuroVue Red and NeuroVue Green and their use for double and triple labeling of neuronal profile. Brain Res Bull 2005;66:249-58.

Fuentemilla L, Marco-Pallarés J, Grau C. Modulation of spectral power and of phase resetting of EEG contributes differentially to the generation of auditory eventrelated potentials. Neuroimage 2006;30:909-16.

Gerfen CR, Sawchenko PE. An anterograde neuroanatomical tracing method that shows the detailed morphology of neurons, their axons and terminals: immunohistochemical localization of an axonally transported plant lectin Phaseolus vulgaris leucoagglutinin (PHA-L). Brain Res 1984;290:219-38.

Gerloff C, Cohen LG, Floeter MK, Chen R, Corwell B, Hallett M. Inhibitory influence of the ipsilateral motor cortex on responses to stimulation of the human cortex and pyramidal tract. J Physiol 1998a;510:249-59.

Gerloff C, Richard J, Hadley J, Schulman AE, Honda M, Hallett M. Functional coupling and regional activation of human cortical motor areas during simple, internally paced and externally paced finger movements. Brain 1998b;121:1513-31.

Gevins AS, Bressler SL, Morgan NH, Cutillo BA, White RM, Greer DS, et al. Eventrelated covariances during a bimanual visuomotor task. I. Methods and analysis of stimulus- and response-locked data. Electroencephalogr Clin Neurophysiol 1989;74:58-75.

Ghosh S, Brinkman C, Porter R. A quantitative study of the distribution of neurons projecting to the precentral motor cortex in the monkey (M. fascicularis). J Comp Neurol 1987;259:424-44.

Ghosh S, Porter R. Corticocortical synaptic influences on morphologically identified pyramidal neurones in the motor cortex of the monkey. J Physiol 1988:400:617-29.

Giambattistelli F, Tomasevic L, Pellegrino G, Porcaro C, Melgari JM, Rossini PM, et al. The spontaneous fluctuation of the excitability of a single node modulates the internodes connectivity: a TMS-EEG study. Hum Brain Mapp 2014;35: 1740-9.
Glover JC, Petursdottir G, Jansen JK. Fluorescent dextran-amines used as axonal tracers in the nervous system of the chicken embryo. I Neurosci Methods $1986 ; 18: 243-54$.

Goldstone RL, Pestilli F, Börner K. Self-portraits of the brain: cognitive science, data visualization, and communicating brain structure and function. Trends Cogn Sci 2015;19:462-74.

Golgi C. Sulla fina anatomia degli organi centrali del sistema nervoso. Reggio Emilia: S. Calderini; 1885

Gosseries O, Sarasso S, Casarotto S, Boly M, Schnakers C, Napolitani M, et al. On the cerebral origin of EEG responses to TMS: insights from severe cortical lesions. Brain Stim 2015;8:142-9.

Groppa S, Schlaak BH, Münchau A, Werner-Petroll N, Dünnweber J, Bäumer T, et al. The human dorsal premotor cortex facilitates the excitability of ipsilateral primary motor cortex via a short latency cortico-cortical route. Hum Brain Mapp 2012;33:419-30.

Haber S. Tracing intrinsic fiber connections in postmortem human brain with WGAHRP. J Neurosci Methods 1988;23:15-22.

Hallett M, Di Iorio R, Rossini PM, Park JE, Chen R, Celnik P, et al. Contribution of transcranial magnetic stimulation to assessment of brain connectivity and networks. Clin Neurophysiol 2017;128:2125-39.

Hämäläinen MS, Ilmoniemi RJ. Interpreting magnetic fields of the brain: minimum norm estimates. Med Biol Eng Comput 1994;32:35-42.

Hannula H, Ilmoniemi RJ. Basic principles of navigated TMS. In: Navigated Transcranial Magnetic Stimulation in Neurosurger. Cham: Springer; 2017. p. 3-29.

Harquel S, Bacle T, Beynel L, Marendaz C, Chauvin A, David O. Mapping dynamical properties of cortical microcircuits using robotized TMS and EEG: towards functional cytoarchitectonics. Neuroimage 2016;135:115-24.

Heilingoetter CL, Jensen MB. Histological methods for ex vivo axon tracing: a systematic review. Neurol Res 2016;38:561-9.

Helfrich RF, Schneider TR, Rach S, Trautmann-Lengsfeld SA, Engel AK, Herrmann CS. Entrainment of brain oscillations by transcranial alternating current stimulation. Curr Biol 2014;24:333-9.

Hess CW, Mills KR, Murray NM. Responses in small hand muscles from magnetic stimulation of the human brain. J Physiol 1987;388:397-419.

Honey CJ, Kötter R, Breakspear M, Sporns O. Network structure of cerebral cortex shapes functional connectivity on multiple time scales. Proc Natl Acad Sci USA 2007; 104:10240-5.

Honey CJ, Sporns O, Cammoun L, Gigandet X, Thiran JP, Meuli R, et al. Predicting human resting-state functional connectivity from structural connectivity. Proc Natl Acad Sci USA 2009;106:2035-40.

Honig MG, Hume RI. Dil and DiO: versatile fluorescent dyes for neuronal labelling and pathway tracing. Trends Neurosci 1989;12:333-41.

Huang D, Ren A, Shang J, Lei Q, Zhang Y, Yin Z, et al. Combining Partial directed coherence and graph theory to analyse effective brain networks of different mental tasks. Front Hum Neurosci 2016;10:235.

Huber R, Maki H, Rosanova M, Casarotto S, Canali P, Casali AG, et al. Human cortical excitability increases with time awake. Cereb Cortex 2013;23:332-8.

Ikeda S, Mizuno-Matsumoto Y, Canuet L, Ishii R, Aoki Y, Hata M, et al. Emotion regulation of neuroticism: emotional information processing related to psychosomatic state evaluated by electroencephalography and exact lowresolution brain electromagnetic tomography. Neuropsychobiology 2015;71:34-41.

Ilic TV, Meintzschel F, Cleff U, Ruge D, Kessler KR, Ziemann U. Short-interval pairedpulse inhibition and facilitation of human motor cortex: the dimension of stimulus intensity. J Physiol 2002;545(1):153-67.

Ilmoniemi RJ, Hernandez-Pavon JC, Mäkelä NN, Metsomaa J, Mutanen TP, Stenroos $M$, et al. Dealing with artifacts in TMS-evoked EEG. In: 37th Annual International Conference of the IEEE Engineering in Medicine and Biology Society (EMBC); 2015. p. 230-33.

Ilmoniemi RJ, Kicic D. Methodology for combined TMS and EEG. Brain Topogr 2010;22:233-48.

Ilmoniemi RJ, Virtanen J, Ruohonen J, Karhu J, Aronen HJ, Näätänen R, et al. Neuronal responses to magnetic stimulation reveal cortical reactivity and connectivity. NeuroReport 1997:8:3537-40.

Ito M, Yoshida M, Obata K, Kawai N, Udo M. Inhibitory control of intracerebellar nuclei by the Purkinje cell axons. Exper Brain Res 1970;10:64-80.

Jahanshahi M, Rothwell J. Transcranial magnetic stimulation studies of cognition: an emerging field. Exp Brain Res 2000;131:1-9.

Jbabdi S, Sotiropoulos SN, Haber SN, Van Essen DC, Behrens TE. Measuring macroscopic brain connections in vivo. Nat Neurosci 2015;18:1546-55.

Jung TP, Makeig S, Westerfield M, Townsend J, Courchesne E, Sejnowski TJ. Analysis and visualization of single-trial event-related potentials. Hum Brain Mapp $2001 ; 14: 166-85$.

Kaarre O, Kallioniemi E, Könönen M, Tolmunen T, Kekkonen V, Kivimäki P, et al. Heavy alcohol use in adolescence is associated with altered cortical activity: a combined TMS-EEG study. Addict Biol 2018;23:268-80.

Kähkönen S, Kesäniemi M, Nikouline VV, Karhu J, Ollikainen M, Holi M, et al. Ethanol modulates cortical activity: direct evidence with combined TMS and EEG. NeuroImage 2001;14:322-8.

Kaminski M, Blinowska K, Szelenberger W. Investigation of coherence structure and EEG activity propagation during sleep. Acta Neurobiol Exp (Wars) 1995;55:213-9.

Kandel A, Buzsaki G. Cellular-synaptic generation of sleep spindles, spike-and-wave discharges, and evoked thalamocortical responses in the neocortex of the rat. J Neurosci 1997;17:6783-97. 
Karabanov AN, Chao C-C, Paine R, Hallett M. Mapping different intra-hemispheric parietal-motor networks using twin coil TMS. Brain Stim 2013;6:384-9.

Kassem MS, Fok SYY, Smith KL, Kuligowski M, Balleine BW. A novel, modernized Golgi-Cox stain optimized for CLARITY cleared tissue. J Neurosci Methods 2017;294:102-10.

Kawaguchi Y, Kondo S. Parvalbumin, somatostatin and cholecystokinin as chemical markers for specific GABAergic interneuron types in the rat frontal cortex. J Neurocytol 2002;31:277-87.

Keil J, Timm J, Sanmiguel I, Schulz H, Obleser J, Schoenwiesner M. Cortical brain states and corticospinal synchronization influence TMS-evoked motor potentials. J Neurophysiol 2014;111:513-9.

Keilholz S, Caballero-Gaudes C, Bandettini P, Deco G, Calhoun V. Time-resolved resting-state functional magnetic resonance imaging analysis: current status, challenges, and new directions. Brain Connect 2017;7:465-81.

Kernell D, Chien-Ping WU. Responses of the pyramidal tract to stimulation of the baboon's motor cortex. J Physiol (Lond) 1967;191:653-72.

Kiebel SJ, David O, Friston KJ. Dynamic causal modelling of evoked responses in EEG/MEG with lead field parameterization. Neuroimage 2006;30:1273-84.

Kiebel SJ, Garrido MI, Moran R, Chen CC, Friston KJ. Dynamic causal modeling for eeg and meg. Hum Brain Mapp 2009;30:1866-76.

Kimiskidis VK, Tsimpiris A, Ryvlin P, Kalviainen R, Koutroumanidis M, Valentin A, et al. TMS combined with EEG in genetic generalized epilepsy: a phase II diagnostic accuracy study. Clin Neurophysiol 2017;128:367-81.

Klamer S, Rona S, Elshahabi A, Lerche H, Braun C, Honegger J, et al. Multimodal effective connectivity analysis reveals seizure focus and propagation in musicogenic epilepsy. Neuroimage 2015;113:70-7.

Koch C, Massimini M, Boly M, Tononi G. Neural correlates of consciousness: progress and problems. Nat Rev Neurosci 2016;17:307-21.

Koch G, Fernandez Del Olmo M, Cheeran B, Ruge D, Schippling S, Caltagirone C, et al. Focal stimulation of the posterior parietal cortex increases the excitability of the ipsilateral motor cortex. J Neurosci 2007a;27:6815-22.

Koch G, Franca M, Mochizuki H, Marconi B, Caltagirone C, Rothwell JC. Interactions between pairs of transcranial magnetic stimuli over the human left dorsal premotor cortex differ from those seen in primary motor cortex. J Physiol 2007b;578:551-62.

Koponen LM, Nieminen JO, Ilmoniemi RJ. Multi-locus transcranial magnetic stimulation-theory and implementation. Brain Stimul 2018. https://doi.org/ 10.1016/i.brs.2018.03.014.

Korhonen RJ, Hernandez-Pavon JC, Metsomaa J, Mäki H, Ilmoniemi RJ, Sarvas J. Removal of large muscle artifacts from transcranial magnetic stimulationevoked EEG by independent component analysis. Med Biol Eng Comput 2011;49:397-407.

Kraskov A, Stogbauer H, Grassberger P. Estimating mutual information. Phys Rev E Stat Nonlin Soft Matter Phys 2004;69:066138.

Kristensson K, Ghetti B, Wiśniewski HM. Study on the propagation of Herpes simplex virus (type 2) into the brain after intraocular injection. Brain Res 1974;69:189-201.

Kristensson K, Olsson Y. Retrograde axonal transport of protein. Brain Res $1971 ; 29: 363-5$.

Kristensson K. Transport of fluorescent protein tracer in peripheral nerves. Acta Neuropathol 1970;16:293-300.

Kubicki S, Herrmann WM, Fichte K, Freund G. Reflections on the topics: EEG frequency bands and regulation of vigilance. Pharmakopsychiatr Neuropsychopharmakol 1979;12:237-45.

Kujirai T, Caramia MD, Rothwell JC, Day BL, Thompson PD, Ferbert A, et al. Corticocortical inhibition in human motor cortex. J Physiol (Lond) 1993;471:501-19.

Kukaswadia S, Wagle-Shukla A, Morgante F, Gunraj C, Chen R. Interactions between long latency afferent inhibition and interhemispheric inhibitions in the human motor cortex. J Physiol 2005;563:915-24.

Kumar S, Sharma A, Tsunoda T. An improved discriminative filter bank selection approach for motor imagery EEG signal classification using mutual information. BMC Bioinf 2017;18:545.

Kuypers HG, Bentivoglio M, Catsman-Berrevoets CE, Bharos AT. Double retrograde neuronal labeling through divergent axon collaterals, using two fluorescent tracers with the same excitation wave length which label different features of the cell. Exp Brain Res 1980;40:383-92.

Kuypers HG, Ugolini G. Viruses as transneuronal tracers. Trends Neurosci 1990;13:71-5.

Lachaux JP, Rodriguez E, Martinerie J, Varela FJ. Measuring phase synchrony in brain signals. Hum Brain Mapp 1999;8:194-208.

Laumann TO, Gordon EM, Adeyemo B, Snyder AZ, Joo SJ, Chen MY, et al. Functional system and areal organization of a highly sampled individual human brain. Neuron 2015;87:657-70.

LaVail JH, LaVail MM. Retrograde axonal transport in the central nervous system. Science 1972;176:1416-7.

Lee JH, Kreitzer AC, Singer AC, Schiff ND. Illuminating neural circuits: from molecules to MRI. J Neurosci 2017;37:10817-25.

Leistedt SJ, Coumans N, Dumont M, Lanquart JP, Stam CJ, Linkowski P. Altered sleep brain functional connectivity in acutely depressed patients. Hum Brain Mapp 2009;30:2207-19.

Lemon RN. Descending pathways in motor control. Annu Rev Neurosci 2008;31:195-218.

Li B, Virtanen JP, Oeltermann A, Schwarz C, Giese MA, Ziemann U, Benali A. Lifting the veil on the dynamics of neuronal activities evoked by transcranial magnetic stimulation. Elife 2017:6. https://doi.org/10.7554/eLife.30552.
Logothetis NK, Pauls J, Augath M, Trinath T, Oeltermann A. Neurophysiological investigation of the basis of the fMRI signal. Nature 2001;412:150-7.

Luppino G, Matelli M, Camarda R, Rizzolatti G. Corticocortical connections of area F3 (SMA-proper) and area F6 (pre-SMA) in the macaque monkey. J Comp Neurol 1993:338:114-40.

Ly JQ Gaggioni G, Chellappa SL, Papachilleos S, Brzozowski A, Borsu C, et al Circadian regulation of human cortical excitability. Nat Commun 2016;7:11828.

Määttä S, Könönen M, Kallioniemi E, Lakka T, Lintu N, Lindi V, et al. Development of cortical motor circuits between childhood and adulthood: a navigated TMSHdEEG study. Hum Brain Mapp 2017;38:2599-615.

Maier-Hein KH, Neher PF, Houde J-C, Côté M-A, Garyfallidis E, Zhong J, et al. The challenge of mapping the human connectome based on diffusion tractography. Nat Commun 2017;8:1349.

Makeig S, Westerfield M, Jung TP, Enghoff S, Townsend J, Courchesne E, et al Dynamic brain sources of visual evoked responses. Science 2002;295:690-4.

Mäki H, Ilmoniemi RJ. EEG oscillations and magnetically evoked motor potentials reflect motor system excitability in overlapping neuronal populations. Clin Neurophysiol 2010;121:492-501.

Mäki H, Ilmoniemi RJ. Projecting out muscle artifacts from TMS-evoked EEG. Neuroimage 2011;54:2706-10.

Mariorenzi R, Zarola F, Caramia MD, Paradiso C, Rossini PM. Non-invasive evaluation of central motor tract excitability changes following peripheral nerve stimulation in healthy humans. Electroencephalogr Clin Neurophysiol 1991;81:90-101.

Markram H, Toledo-Rodriguez M, Wang Y, Gupta A, Silberberg G, Wu C. Interneurons of the neocortical inhibitory system. Nat Rev Neurosci 2004;5:793-807.

Massimini M, Ferrarelli F, Huber R, Esser SK, Singh H, Tononi G. Breakdown of cortical effective connectivity during sleep. Science 2005;309:2228-32.

Massimini M, Ferrarelli F, Murphy M, Huber R, Riedner B, Casarotto S, et al. Cortical reactivity and effective connectivity during REM sleep in humans. Cogn Neurosci 2010;1:176-83.

McDonnell MN, Orekhov Y, Ziemann U. The role of GABA(B) receptors in intracortical inhibition in the human motor cortex. Exp Brain Res 2006;173:86-93.

McIntosh AR, Bratislav M. Multivariate statistical analyses for neuroimaging data Annu Rev Psychol 2013;64:499-525.

Mesulam MM. Tracing neural connections of human brain with selective silver impregnation. Observations on geniculocalcarine, spinothalamic, and entorhinal pathways. Arch Neurol 1979;36:814-8.

Miraglia F, Vecchio F, Bramanti P, Rossini PM. EEG characteristics in "eyes-open" versus "eyes-closed" conditions: Small-world network architecture in healthy aging and age-related brain degeneration. Clin Neurophysiol 2016;127:1261-8.

Miraglia F, Vecchio F, Bramanti P, Rossini PM. Small-worldness characteristics and its gender relation in specific hemispheric networks. Neuroscience 2015;310:1-11.

Miraglia F, Vecchio F, Rossini PM. Searching for signs of aging and dementia in EEC through network analysis. Behav Brain Res 2017;317:292-300.

Mochizuki H, Huang YZ, Rothwell JC. Interhemispheric interaction between human dorsal premotor and contralateral primary motor cortex. J Physiol 2004;561:331-8.

Moharramipour A, Mostame P, Hossein-Zadeh GA, Wheless JW, Babajani-Feremi A Comparison of statistical tests in effective connectivity analysis of ECoG data. Neurosci Methods 2018;308:317-29.

Morawski M, Kirilina E, Scherf N, Jäger C, Reimann K, Trampel R, et al. Developing 3D microscopy with CLARITY on human brain tissue: Towards a tool for informing and validating MRI-based histology. Neuroimage 2018;182:417-28.

Mori S, Setsu W, Nagae-Poetscher LM, Van Zijl PCM. Three-dimensional atlas of brain white matter tracts. MRI Atlas Human White Matter 2005:15-31.

Morishima Y, Akaishi R, Yamada Y, Okuda J, Toma K, Sakai K. Task-specific signal transmission from prefrontal cortex in visual selective attention. Nat Neurosci 2009;12:85-91.

Muakkassa KF, Strick PL. Frontal lobe inputs to primate motor cortex: evidence for four somatotopically organized 'premotor' areas. Brain Res 1979;177:176-82.

Mueller JK, Grigsby EM, Prevosto V, Petraglia 3rd FW, Rao H, Deng ZD, et al Simultaneous transcranial magnetic stimulation and single-neuron recording in alert non-human primates. Nat Neurosci 2014;17:1130-6.

Müller-Dahlhaus JFM, Liu Y, Ziemann U. Inhibitory circuits and the nature of their interactions in the human motor cortex - a pharmacological TMS study. J Physiol 2008;586:495-514.

Mutanen T, Mäki H, Ilmoniemi RJ. The effect of stimulus parameters on TMS-EEG muscle artifacts. Brain Stim 2013;6:371-6.

Mutanen TP, Metsomaa J, Liljander S, Ilmoniemi RJ. Automatic and robust noise suppression in EEG and MEG: the SOUND algorithm. Neuroimage 2018:166:135-51.

Nauta WJH, Gygax PA. Silver impregnation of degenerating axon terminals in the central nervous system: (1) technic (2) chemical notes. Stain Technol $1951 ; 26: 3-9$.

Nguyen VT, Breakspear M, Cunnington R. Fusing concurrent EEG-fMRI with dynamic causal modeling: application to effective connectivity during face perception. Neuroimage 2014;102:60-70.

Ni Z, Gunraj C, Nelson AJ, Yeh IJ, Castillo G, Hoque T, et al. Two phases of interhemispheric inhibition between motor related cortical areas and the primary motor cortex in human. Cereb Cortex 2009;19:1654-65.

Niedermeyer E, da Silva FL. Electroencephalography: Basic Principles, Clinical Applications, and Related Fields. Lippincott Williams \& Wilkins; 2005. 
Nieminen JO, Koponen LM, Mäkelä N, Ilmoniemi RJ. Multi-locus transcranial magnetic stimulation of the primary motor cortex. Brain Stim 2017;10:443.

Nitsche MA, Cohen LG, Wassermann EM, Priori A, Lang N, Antal A, et al. Transcrania direct current stimulation: state of the art 2008. Brain Stim 2008;1:206-23.

Nitsche MA, Doemkes S, Karakoese T, Antal A, Liebetanz D, Lang N, et al. Shaping the effects of transcranial direct current stimulation of the human motor cortex. J Neurophysiol 2007;97:3109-17.

Oh MS, Hong SJ, Huh Y, Kim K-S. Expression of transgenes in midbrain dopamine neurons using the tyrosine hydroxylase promoter. Gene Ther 2009:16:437-40.

Oliveri M, Babiloni C, Filippi MM, Caltagirone C, Babiloni F, Cicinelli P, et al. Influence of the supplementary motor area on primary motor cortex excitability during movements triggered by neutral or emotionally unpleasant visual cues. Exp Brain Res 2003;149:214-21.

Oliveri M, Rossini PM, Filippi MM, Traversa R, Cicinelli P, Palmieri MG, et al. Timedependent activation of parieto-frontal networks for directing attention to tactile space. A study with paired transcranial magnetic stimulation pulses in right-brain-damaged patients with extinction. Brain 2000;123:1939-47.

Oliveri M, Rossini PM, Pasqualetti P, Traversa R, Cicinelli P, Palmieri MG, et al. Interhemispheric asymmetries in the perception of unimanual and bimanual cutaneous stimuli. A study using transcranial magnetic stimulation. Brain 1999; 122:1721-9.

Onnela JP, Saramaki J, Kertesz J, Kaski K. Intensity and coherence of motifs in weighted complex networks. Phys Rev E Stat Nonlin Soft Matt Phys 2005;71:065103.

Parker GJM, Stephan KE, Barker GJ, Rowe JB, MacManus DG, Wheeler-Kingshott CAM, et al. Initial demonstration of in vivo tracing of axonal projections in the macaque brain and comparison with the human brain using diffusion tensor imaging and fast marching tractography. Neuroimage 2002;15:797-809.

Pascual-Marqui RD, Lehmann D, Koukkou M, Kochi K, Anderer P, Saletu B, et al. Assessing interactions in the brain with exact low-resolution electromagnetic tomography. Philos Trans A Math Phys Eng Sci 2011;369:3768-84.

Pascual-Marqui RD. Discrete, 3D Distributed, Linear Imaging Methods of Electric Neuronal Activity. Part 1: Exact, Zero Error Localization. 2007a; arXiv:0710.3341.

Pascual-Marqui RD. Instantaneous and lagged measurements of linear and nonlinear dependence between groups of multivariate time series: frequency decomposition. 2007b; arXiv:0711.1455.

Pascual-Marqui RD. Standardized low-resolution brain electromagnetic tomography (sLORETA): technical details. Methods Find Exp Clin Pharmaco 2002;24(Suppl D):5-12.

Pascual-Marqui RD. Theory of the EEG Inverse Problem. In: Thakor Nitish V, Tong Shanbao, editors. Quantitative EEG Analysis: Methods and Clinical Applications. Artech House. p. 121-40.

Paus T. Inferring causality in brain images: a perturbation approach. Philos Trans R Soc Lond B Biol Sci 2005;360:1109-14.

Penny WD, Stephan KE, Mechelli A, Friston KJ. Modelling functional integration: a comparison of structural equation and dynamic causal models. Neuroimage 2004;23(Suppl 1):S264-74.

Penttonen M, Nurminen N, Miettinen R, Sirvio J, Henze DA, Csicsvari J, et al. Ultraslow oscillation $(0.025 \mathrm{~Hz})$ triggers hippocampal afterdischarges in Wistar rats. Neuroscience 1999;94:735-43.

Pestilli F, Yeatman JD, Rokem A, Kay KN, Wandell BA. Evaluation and statistical inference for human connectomes. Nat Methods 2014;11:1058-63.

Pestilli F. Test-retest measurements and digital validation for in vivo neuroscience. Sci Data 2015;2:140057.

Petro NM, Gruss LF, Yin S, Huang H, Miskovic V, Ding M, et al. Multimodal imaging evidence for a frontoparietal modulation of visual cortex during the selective processing of conditioned threat. J Cogn Neurosci 2017;29:953-67.

Pisoni A, Mattavelli G, Papagno C, Rosanova M, Casali AG, Romero Lauro LJ. Cognitive enhancement induced by anodal tDCS drives circuit-specific cortical plasticity. Cereb Cortex 2018;28:1132-40.

Polania R, Nitsche MA, Korman C, Batsikadze G, Paulus W. The importance of timing in segregated theta phase-coupling for cognitive performance. Curr Biol 2012a;22:1314-8.

Polania R, Nitsche MA, Paulus W. Modulating functional connectivity patterns and topological functional organization of the human brain with transcranial direct current stimulation. Hum Brain Mapp 2011a;32:1236-49.

Polania R, Paulus W, Antal A, Nitsche MA. Introducing graph theory to track for neuroplastic alterations in the resting human brain: a transcranial direct current stimulation study. Neuroimage 2011b;54:2287-96.

Polania R, Paulus W, Nitsche MA. Modulating cortico-striatal and thalamo-cortica functional connectivity with transcranial direct current stimulation. Hum Brain Mapp 2012b;33:2499-508.

Premoli I, Biondi A, Carlesso S, Rivolta D, Richardson MP. Lamotrigine and levetiracetam exert a similar modulation of TMS-evoked EEG potentials. Epilepsia 2017;58:42-50.

Premoli I, Castellanos N, Rivolta D, Belardinelli P, Bajo R, Zipser C, et al. TMS-EEC signatures of GABAergic neurotransmission in the human cortex. J Neurosci 2014;34:5603-12.

Ptak R, Schnider A, Fellrath J. The dorsal frontoparietal network: a core system for emulated action. Trends Cogn Sci 2017;21:589-99.

Quessy S, Côté SL, Hamadjida A, Deffeyes J, Dancause N. Modulatory effects of the ipsi and contralateral ventral premotor cortex (PMv) on the primary motor cortex (M1) outputs to intrinsic hand and forearm muscles in Cebus apella. Cereb Cortex 2016;26:3905-20.
Ragazzoni A, Pirulli C, Veniero D, Feurra M, Cincotta M, Giovannelli F, et al. Vegetative versus minimally conscious states: a study using TMS-EEG, sensory and event-related potentials. PloS One 2013;8:e57069.

Raichle ME. The brain's default mode network. Ann Rev Neurosci 2015;38:433-47.

Ramyead A, Kometer M, Studerus E, Koranyi S, Ittig S, Gschwandtner U, et al. Aberrant current source-density and lagged phase synchronization of neural oscillations as markers for emerging psychosis. Schizophr Bull 2015;41:919-29.

Rehme AK, Eickhoff SB, Wang LE, Fink GR, Grefkes C. Dynamic causal modeling of cortical activity from the acute to the chronic stage after stroke. Neuroimage 2011;55:1147-58

Risacher SL, Saykin AJ. Neuroimaging biomarkers of neurodegenerative diseases and dementia. Semin Neurol 2013:33:386-416.

Rivera-Urbina GN, Batsikadze G, Molero-Chamizo A, Paulus W, Kuo MF, Nitsche MA. Parietal transcranial direct current stimulation modulates primary motor cortex excitability. Eur J Neurosci 2015;41:845-55.

Robertson EM, Théoret H, Pascual-Leone A. Studies in cognition: the problems solved and created by transcranial magnetic stimulation. J Cogn Neurosci 2003;15:948-60.

Rogasch NC, Fitzgerald PB. Assessing cortical network properties using TMS-EEG. Hum Brain Mapp 2013;34:1652-69.

Rogasch NC, Thomson RH, Farzan F, Fitzgibbon BM, Bailey NW, Hernandez-Pavon JC, et al. Removing artefacts from TMS-EEG recordings using independent component analysis: importance for assessing prefrontal and motor cortex network properties. Neuroimage 2014;101:425-39.

Rokem A, Takemura H, Bock AS, Scherf KS, Behrmann M, Wandell BA, et al. The visual white matter: the application of diffusion MRI and fiber tractography to vision science. J Vis 2017;17:4.

Rosanova M, Casali A, Bellina V, Resta F, Mariotti M, Massimini M. Natural frequencies of human corticothalamic circuits. J Neurosci 2009;29:7679-85.

Rosanova M, Fecchio M, Casarotto S, Sarasso S, Casali AG, Pigorini A, et al. Sleep-like cortical OFF-periods disrupt causality and complexity in the brain of unresponsive wakefulness syndrome patients. Nat Commun 2018;9:4427.

Rosanova M, Gosseries O, Casarotto S, Boly M, Casali AG, Bruno MA, et al. Recovery of cortical effective connectivity and recovery of consciousness in vegetative patients. Brain 2012;135:1308-20.

Rossi S, Cappa SF, Babiloni C, Pasqualetti P, Miniussi C, Carducci F, et al. Prefrontal [correction of Prefontal] cortex in long-term memory: an "interference" approach using magnetic stimulation.. Nat Neurosci 2001;4:948-52. Erratum in: Nat Neurosci 2002; 5:1017.

Rossi S, Miniussi C, Pasqualetti P, Babiloni C, Rossini PM, Cappa SF. Age-related functional changes of prefrontal cortex in long-term memory: a repetitive transcranial magnetic stimulation study. J Neurosci 2004;24:7939-44.

Rossini PM, Burke D, Chen R, Cohen LG, Daskalakis Z, Di Iorio R, et al. Non-invasive electrical and magnetic stimulation of the brain, spinal cord, roots and peripheral nerves: Basic principles and procedures for routine clinical and research application. An updated report from an I.F.C.N. Committee. Clin Neurophysiol 2015:126:1071-107.

Rossini PM, Caramia MD, Iani C, Desiato MT, Sciarretta G, Bernardi G. Magnetic transcranial stimulation in healthy humans: influence on the behavior of upper limb motor units. Brain Res 1995;676:314-24.

Roth BL. DREADDs for neuroscientists. Neuron 2016:89:683-94.

Rubinov M, Sporns O. Complex network measures of brain connectivity: uses and interpretations. Neuroimage 2010;52:1059-69.

Sakai K, Ugawa Y, Terao Y, Hanajima R, Furubayashi T, Kanazawa I. Preferential activation of different I-waves by transcranial magnetic stimulation with a figure-of-eight-shaped coil. Exp Brain Res 1997;113:24-32.

Sarasso S, Boly M, Napolitani M, Gosseries O, Charland-Verville V, Casarotto S, et al. Consciousness and complexity during unresponsiveness induced by propofol, xenon, and ketamine. Curr Biol 2015;25:3099-105.

Schmahmann JD, Pandya DN, Wang R, Dai G, D'Arceuil HE, de Crespigny AJ, et al. Association fiber pathways of the brain: parallel observations from diffusion spectrum imaging and autoradiography. Brain 2007;130:630-53.

Schmahmann JD, Pandya DN. Cerebral white matter-historical evolution of facts and notions concerning the organization of the fiber pathways of the brain. Hist Neurosci 2007:16:237-67.

Schmued LC, Fallon JH. Fluoro-gold: a new fluorescent retrograde axonal tracer with numerous unique properties. Brain Res 1986;377:147-54.

Schoffelen JM, Gross J. Source connectivity analysis with MEG and EEG. Hum Brain Mapp 2009;30:1857-65.

Scholvinck ML, Maier A, Ye FQ, Duyn JH, Leopold DA. Neural basis of global restingstate fMRI activity. Proc Natl Acad Sci USA 2010;107:10238-43.

Seeck M, Koessler L, Bast T, Leijten F, Michel C, Baumgartner C, et al. The standardized EEG electrode array of the IFCN. Clin Neurophysiol 2017;128:2070-7.

Shepherd GM. Foundations of the Neuron Doctrine: 25th Anniversary Edition. Oxford University Press 2015.

Shirota Y, Hamada M, Terao Y, Ohminami S, Tsutsumi R, Ugawa Y, et al. Increased primary motor cortical excitability by a single-pulse transcranial magnetic stimulation over the supplementary motor area. Exp Brain Res 2012;219:339-49.

Siebner HR, Bergmann TO, Bestmann S, Massimini M, Johansen-Berg H, Mochizuki $\mathrm{H}$, et al. Consensus paper: Combining transcranial stimulation with neuroimaging. Brain Stim 2009;2:58-80.

Singer W, Gray CM. Visual feature integration and the temporal correlation hypothesis. Ann Rev Neurosci 1995;18:555-86. 
Singer W. Neurobiology. Striving for coherence [news; comment]. Nature 1999a;397(391):3.

Singer W. The formation of cooperative cell assemblies in the visual cortex. J Exp Biol 1990;153:177-97.

Singer W. Time as coding space? Curr Opin Neurobio 1999b;9:189-94.

Smith SM, Hyvärinen A, Varoquaux G, Miller KL, Beckmann CF. Group-PCA for very large fMRI datasets. Neuroimage 2014;101:738-49.

Smith SM, Nichols TE, Vidaurre D, Winkler AM, Behrens TE, Glasser MF, et al. A positive-negative mode of population covariation links brain connectivity, demographics and behavior. Nat Neurosci 2015;18:1565-7.

Sowell ER, Peterson BS, Thompson PM, Welcome SE, Henkenius AL, Toga AW. Mapping cortical change across the human life span. Nat Neurosci 2003;6:309-15.

Sporns O, Zwi JD. The small world of the cerebral cortex. Neuroinformatics 2004;2:145-62.

Sporns O. Discovering the Human Connectome. MIT Press; 2012.

Sporns O. The human connectome: a complex network. Ann NY Acad Sci 2011;1224:109-25.

Sporns 0. The human connectome: origins and challenges. Neuroimage 2013;80:53-61.

Stagg CJ, Nitsche MA. Physiological basis of transcranial direct current stimulation. Neuroscientist 2011;17:37-53.

Stam CJ, de Haan W, Daffertshofer A, Jones BF, Manshanden I, van Cappellen, van Walsum AM, et al. Graph theoretical analysis of magnetoencephalographic functional connectivity in Alzheimer's disease. Brain 2009;132:213-24.

Stam CJ, Jones BF, Nolte G, Breakspear M, Scheltens P. Small-world networks and functional connectivity in Alzheimer's disease. Cereb Cortex 2007;17:92-9.

Stam CJ. Modern network science of neurological disorders. Nat Rev Neurosci 2014; 15:683-95.

Stepniewska I, Preuss TM, Kaas JH. Architectionis, somatotopic organization, and ipsilateral cortical connections of the primary motor area (M1) of owl monkeys. J Comp Neurol 1993;330:238-71.

Strobel SA. Ribozyme chemogenetics. Biopolymers 1998;48:65-81.

Takemura H, Caiafa CF, Wandell BA, Pestilli F. Ensemble tractography. PLoS Comput Biol 2016a;12:e1004692.

Takemura H, Rokem A, Winawer J, Yeatman JD, Wandell BA, Pestilli F. A major human white matter pathway between dorsal and ventral visual cortex. Cereb Cortex 2016b;26:2205-14.

Talairach J, Tournoux P. Co-planar Stereotaxic Atlas of the Human Brain. New York: Thieme Medical; 1988.

Terao Y, Ugawa Y, Enomoto H, Furubayashi T, Shiio Y, Machii K, et al. Hemispheric lateralization in the cortical motor preparation for human vocalization. J Neurosci 2001;21:1600-9.

Thomas C, Ye FQ Irfanoglu MO, Modi P, Saleem KS, Leopold DA, et al. Anatomical accuracy of brain connections derived from diffusion MRI tractography is inherently limited. Proc Natl Acad Sci USA 2014;111:16574-9.

Tokimura H, Di Lazzaro V, Tokimura Y, Oliviero A, Profice P, Insola A, et al. Short latency inhibition of human hand motor cortex by somatosensory input from the hand. J Physiol 2000;523:503-13.

Tokimura H, Ridding MC, Tokimura Y, Amassian VE, Rothwell JC. Short latency facilitation between pairs of threshold magnetic stimuli applied to human motor cortex. Electroencephalogr Clin Neurophysiol 1996;101:263-72.

Tokuno H, Nambu A. Organization of nonprimary motor cortical inputs on pyramidal and nonpyramidal tract neurons of primary motor cortex: an electrophysiological study in the macaque monkey. Cereb Cortex 2000;10:58-68.

Tononi G, Sporns O, Edelman GM. A measure for brain complexity: relating functional segregation and integration in the nervous system. Proc Natl Acad Sci USA 1994;91:5033-7.

Triesch J, Zrenner C, Ziemann U. Modeling TMS-induced I-waves in human motor cortex. Prog Brain Res 2015;222:105-24.

Tsutsumi R, Shirota Y, Ohminami S, Terao Y, Ugawa Y, Hanajima R. Conditioning intensity-dependent interaction between short-latency interhemispheric inhibition and short-latency afferent inhibition. J Neurophysiol 2012;108:1130-7.

Ugawa Y, Day BL, Rothwell JC, Thompson PD, Merton PA, Marsden CD. Modulation of motor cortical excitability by electrical stimulation over the cerebellum in man. J Physiol 1991;441:57-72.

Ugawa Y, Genba-Shimizu K, Rothwell JC, Iwata M, Kanazawa I. Suppression of motor cortical excitability by electrical stimulation over the cerebellum in ataxia. Ann Neurol 1994;36:90-6.

Ugawa Y, Terao Y, Hanajima R, Sakai K, Furubayashi T, Machii K, Kanazawa I. Magnetic stimulation over the cerebellum in patients with ataxia. Electroenceph Clin Neurophysiol 1997;104:453-8.

Ugawa Y, Uesaka Y, Terao Y, Hanajima R, Kanazawa I. Magnetic stimulation over the cerebellum in humans. Ann Neurol 1995;37:703-13.

Valls-Sole J, Pascual-Leone A, Wassermann EM, Hallett M. Human motor evoked responses to paired transcranial magnetic stimuli. Electroencephalogr Clin Neurophysiol 1992;85:355-64.

van den Broek SP, Reinders F, Donderwinkel M, Peters MJ. Volume conduction effects in EEG and MEG. Electroencephalogr Clin Neurophysiol 1998;106:522-34.

van den Heuvel MP, Hulshoff Pol HE. Exploring the brain network: a review on resting-state fMRI functional connectivity. Eur Neuropsychopharmacol 2010;20:519-34.
Vecchio F, Miraglia F, Bramanti P, Rossini PM. Human brain networks in physiological aging: a graph theoretical analysis of cortical connectivity from EEG data. J Alzheimers Dis 2014a;41:1239-49.

Vecchio F, Miraglia F, Marra C, Quaranta D, Vita MG, Bramanti P, et al. Human brain networks in cognitive decline: a graph theoretical analysis of cortical connectivity from EEG data. J Alzheimers Dis 2014b;41:113-27.

Vecchio F, Miraglia F, Piludu F, Granata G, Romanello R, Caulo M, et al. "Small World" architecture in brain connectivity and hippocampal volume in Alzheimer's disease: a study via graph theory from EEG data. Brain Imag Behav 2017;11:473-85.

Vecchio F, Miraglia F, Quaranta D, Granata G, Romanello R, Marra C, et al. Cortical connectivity and memory performance in cognitive decline: a study via graph theory from EEG data. Neuroscience 2016;316:143-50.

Vecchio F, Miraglia F, Valeriani L, Scarpellini MG, Bramanti P, Mecarelli O, et al. Cortical brain connectivity and B-type natriuretic peptide in patients with congestive heart failure. Clin EEG Neurosci 2015;46:224-9.

Vecchio F, Miraglia F, Rossini PM. Tracking neuronal connectivity from electric brain signals to predict performance. Neuroscientist 2018. 1073858418776891.

Virtanen J, Ruohonen J, Näätänen R, Ilmoniemi RJ. Instrumentation for the measurement of electric brain responses to transcranial magnetic stimulation. Med Biol Eng Comput 1999;37:322-6.

Volz LJ, Sarfeld AS, Diekhoff S, Rehme AK, Pool EM, Eickhoff SB, et al. Motor cortex excitability and connectivity in chronic stroke: a multimodal model of functional reorganization. Brain Struct Funct 2015;220:1093-107.

von Stein A, Sarnthein J. Different frequencies for different scales of cortical integration: from local gamma to long range alpha/theta synchronization. Int J Psychophysiol 2000;38:301-13.

Voss U, Holzmann R, Hobson A, Paulus W, Koppehele-Gossel J, Klimke A, et al. Induction of self awareness in dreams through frontal low current stimulation of gamma activity. Nat Neurosci 2014;17:810-2.

Wahl M, Lauterbach-Soon B, Hattingen E, Hubers A, Ziemann U. Callosal anatomical and effective connectivity between primary motor cortices predicts visually cued bimanual temporal coordination performance. Brain Struct Funct 2016;221:3427-43.

Wahl M, Lauterbach-Soon B, Hattingen E, Jung P, Singer O, Volz S, et al. Human motor corpus callosum: topography, somatotopy, and link between microstructure and function. J Neurosci 2007;27:12132-8.

Wandell BA, Le RK. Diagnosing the neural circuitry of reading. Neuron 2017;96:298-311.

Walsh V, Cowey A. Transcranial magnetic stimulation and cognitive neuroscience. Nat Rev Neurosci 2000;1:73-9.

Walsh V, Desmond JE, Pascual-Leone A. Manipulating brains. Behav Neurol 2006;17:131-4.

Wedeen VJ, Hagmann P, Tseng W-YI, Reese TG, Weisskoff RM. Mapping complex tissue architecture with diffusion spectrum magnetic resonance imaging. Magn Reson Med 2005;54:1377-86.

Werhahn KJ, Taylor J, Ridding M, Meyer BU, Rothwell JC. Effect of transcranial magnetic stimulation over the cerebellum on the excitability of human motor cortex. Electroencephalogr Clin Neurophysiol 1996;101:58-66.

Westlye LT, Walhovd KB, Dale AM, Bjørnerud A, Due-Tønnessen P, Engvig A, et al. Life-span changes of the human brain white matter: diffusion tensor imaging (DTI) and volumetry. Cereb Cortex 2009;20:2055-68.

Worsley KJ, Chen JI, Lerch J, Evans AC. Comparing functional connectivity via thresholding correlations and singular value decomposition. Philos Trans R Soc Lond B Biol Sci 2005;360:913-20.

Xiong J, Parsons LM, Gao JH, Fox PT. Interregional connectivity to primary motor cortex revealed using MRI resting state images. Hum Brain Mapp 1999;8:151-6.

Yeatman JD, Dougherty RF, Myall NJ, Wandell BA, Feldman HM. Tract profiles of white matter properties: automating fiber-tract quantification. PLoS ONE 2012; 7:e49790.

Zhou C, Zemanová L, Zamora G, Hilgetag CC, Kurths J. Hierarchical organization unveiled by functional connectivity in complex brain networks. Phys Rev Lett 2006;97:238103.

Ziemann U, Reis J, Schwenkreis P, Rosanova M, Strafella A, Badawy R, et al. TMS and drugs revisited 2014. Clin Neurophysiol 2015;126:1847-68.

Ziemann U, Rothwell JC, Ridding MC. Interaction between intracortical inhibition and facilitation in human motor cortex. J Physiol 1996;496:873-81.

Ziemann U, Rothwell JC. I-waves in motor cortex. J Clin Neurophysiol 2000; 17:397-405.

Ziemann U, Tergau F, Wassermann EM, Wischer S, Hildebrandt J, Paulus W. Demonstration of facilitatory I-wave interaction in the human motor cortex by paired transcranial magnetic stimulation. J Physiol 1998;511:181-90.

Ziemann U. Transcranial magnetic stimulation at the interface with other techniques: a powerful tool for studying the human cortex. Neuroscientist 2011;17:368-81.

Zrenner C, Desideri D, Belardinelli P, Ziemann U. Real-time EEG-defined excitability states determine efficacy of TMS-induced plasticity in human motor cortex. Brain Stimul 2018;11:374-89.

Zuo XN, Xing XX. Test-retest reliabilities of restingstate FMRI measurements in human brain functional connectomics: a systems neuroscience perspective. Neurosci Biobehav Rev 2014;45:100-18. 